\title{
32. DISTRIBUTION OF MICROPERFORATE TENUITELLID PLANKTONIC FORAMINIFERS IN HOLES 747A AND 749B, KERGUELEN PLATEAU ${ }^{1}$
}

\author{
Qianyu Li, ${ }^{2}$ Sally S. Radford, ${ }^{2}$ and Fred T. Banner ${ }^{3}$
}

\begin{abstract}
Late Eocene to Pleistocene planktonic foraminifers from Leg 120 Holes 747A and 749B on the Kerguelen Plateau were quantitatively analyzed. Microperforate tenuitellid forms dominate the Oligocene to middle Miocene, and 17 species (including the new species Tenuitella jamesi and Tenuitellinata selleyi) are recorded.

A lineage zonation of tenuitellid foraminifers is proposed as an alternative scheme for refinement of the Oligocene-Miocene biostratigraphy in high latitudes. Progressive or abrupt alterations in morphological characters within this lineage, producing different morphotypes or species, coincided with prolonged or sudden changes in paleoclimate. These microperforate planktonic foraminifers thus appear to have potential as indicators of cold-water masses and temperature fluctuations in post-Eocene oceans.
\end{abstract}

\section{INTRODUCTION}

Microperforate planktonic foraminifers of the post-Eocene are characterized by a pustulate wall with perforations $<1 \mu \mathrm{m}$ in diameter. Fleisher (1974) first distinguished these forms as Tenuitella, with a primitive, microperforate, rather than macroperforate or spinose, wall texture. Previously assigned mainly to Globigerina or Globorotalia, these tenuitellids received little attention in taxonomic or biostratigraphic studies because of their small test size (mostly $100-150 \mu \mathrm{m}$ ). Li (1987) first investigated their evolution in the Oligocene-Miocene of Trinidad, and Brummer (1988) studied modern species from the Indian Ocean.

The group became quite diverse throughout the Oligocene and Miocene and the primitive wall texture is of value in evolutionary studies. Systematic information on high-latitude Oligocene-Miocene microperforate species remains sparse, however, although they have long been known to be the most common components there (e.g., Subbotina, 1953; Fleisher, 1974, 1975; Poore, 1979). This consequently hinders a better understanding of their potential biostratigraphic and paleoceanographic significance.

Leg 120 drilled five sites on the Kerguelen Plateau in the southern Indian Ocean and recovered almost complete Cenozoic sequences rich in high-latitude microfaunas (Schlich, Wise, et al., 1989). Holes 747A and 749B were selected for this study to record the distribution and biostratigraphy of the microperforate species.

Hole 747A is located in the transition zone between the Northern and Southern Kerguelen Plateaus, whereas Hole 749 lies on the western flank of the Southern Kerguelen Plateau. Table 1 summarizes the drilling results.

\section{MATERIALS AND METHODS}

Of 137 samples studied, 98 are from Hole 747A (Oligocene to Pleistocene) and 39 from Hole 749B (middle Eocene to Oligocene). They were collected mostly at intervals of $2-3 \mathrm{~m}$,

\footnotetext{
${ }^{1}$ Wise, S. W., Jr., Schlich, R., et al., 1992. Proc. ODP, Sci. Results, 120: College Station, TX (Ocean Drilling Program).

2 Department of Geology, Imperial College, Prince Consort Road, London SW7 2BP, United Kingdom.

${ }^{3}$ Department of Palaeontology, Natural History Museum, Cromwell Road, London SW7 5BD, United Kingdom.
}

but some were taken at about $20-\mathrm{cm}$ intervals across major stratigraphic boundaries.

Samples were processed by standard washing and drying. Residues were then separated through a $150-\mu \mathrm{m}$ sieve into A $(>150 \mu \mathrm{m})$ and $\mathrm{B}(<150-63 \mu \mathrm{m})$ fractions. Planktonic foraminifers were examined first with binocular microscopes and then with a Hitachi 2500 scanning electron microscope (SEM). The first 150 or more specimens of planktonic foraminifers from each fraction (i.e., at least 300 from each sample) were counted. The Eocene samples (except those from the uppermost Eocene) from Hole 749B, were not, however, quantitatively analyzed as they lack tenuitellid species relevant to this study. Quantitative data on planktonic foraminifers are presented in Tables 2 and 3.

Preservation of specimens is generally excellent, although some large forms show overgrowth or recrystallization in samples that contain common radiolarians.

\section{REVISION OF PLANKTONIC FORAMINIFER BIOSTRATIGRAPHY}

The Cenozoic planktonic foraminifer zonation for Holes $747 \mathrm{~A}$ and $749 \mathrm{~B}$, as established by shipboard micropaleontologists in site reports (Schlich, Wise, et al., 1989), coupled with the Antarctic zonation scheme of Stott and Kennett (1990), were used as the basis for biostratigraphic correlation. During the course of this study, however, macroperforate as well as microperforate species were examined, resulting in modification of these zonations, as summarized below. Our results are similar to those of Berggren (Chapters 31 and 35, this volume), although disagreements exist because of different species concepts employed.

\section{Hole 747A}

Because of poor recovery, the lowermost Oligocene is missing from Hole 747A (Table 4 and Fig. 1). The Paleocene and Eocene were strongly condensed and interrupted by several hiatuses. Only the Subbotina trinidadensis Zone (P1C of Berggren and Miller, 1988) is identified from the Paleocene. No Eocene zones were defined, but Eocene faunas were mixed with forms of Paleocene age (below) and Oligocene age (above).

The Subbotina euapertura Zone, with its Chiloguembelina cubensis and $S$. labiacrassata subzones, includes almost the entire upper Oligocene and part of the lower Oligocene. The 
Table 1. Summary of drilling results.

\begin{tabular}{|c|c|c|c|c|}
\hline Hole & Location & $\begin{array}{l}\text { Water depth } \\
\text { (m) }\end{array}$ & $\begin{array}{l}\text { Penetration/ } \\
\text { recovery }\end{array}$ & Sediments recovered $(\mathrm{m})$ \\
\hline $747 \mathrm{~A}$ & $\begin{array}{l}54^{\circ} 48.65^{\prime} \mathrm{S} \\
76^{\circ} 47.64^{\prime} \mathrm{E}\end{array}$ & 1695 & $256 / 227.34$ & $\begin{array}{l}\text { Pliocene-Pleistocene ( } 35) \\
\text { Miocene }(92) \\
\text { Oligocene }(43) \\
\text { Maestrichtian-Eocene ( } 86)\end{array}$ \\
\hline $749 B$ & $\begin{array}{l}58^{\circ} 43.03^{\prime} \mathrm{S} \\
76^{\circ} 24.45^{\prime} \mathrm{E}\end{array}$ & 1069.5 & $123.8 / 64.66$ & $\begin{array}{l}\text { Pliocene-Pleistocene }(0.3) \\
\text { Oligocene }(15) \\
\text { Eocene }(108.5)\end{array}$ \\
\hline
\end{tabular}

Note: Data from Schlich, Wise, et al. (1989).

contact of these two subzones denotes the lower/upper Oligocene boundary. In the upper Oligocene, however, Berggren (Chapter 31, this volume) distinguished, from bottom to top, the Globigerina labiacrassata, G. euapertura, and G. brazieri zones (part) (Fig. 1).

The Paragloborotalia incognita/P. semivera Zone of the lower Miocene was identified on the common appearance of $P$. incognita (including $P$. pseudocontinuosa). It was recognized by Berggren et al. (1983) as Zone M2 from the South Atlantic, with typical zonal markers. It is equivalent to the upper $G$. brazieri and $P$. incognita zones of Berggren (Chapter 35 , this volume). In Hole $747 \mathrm{~A}, P$. semivera is always rare and atypical, but this zone also contains the equally macroperforate, cancellate Globigerina woodi, although the specimens are slightly smaller $(180-200 \mu \mathrm{m})$ than typical ones from younger sequences in the $G$. woodi-G. praescitula Zone.

Similar caution should also be exercised in the recognition of the Globorotalia zealandica/G. pseudomiozea Zone in the upper part of the lower Miocene, because G. zealandica, if present at all, is never typical, compared with those figured in Berggren et al. (1983). In contrast, Globorotalia praescitula is rather common, which led Berggren (Chapter 35 , this volume) to recognize the $G$. praescitula Zone for this interval.

The Globigerina woodi-Globorotalia praescitula Zone in the middle Miocene has been identified, but from a level slightly lower than that in the site report. It is equivalent to the Globorotalia miozea and lower Neogloboquadrina nympha zones in Berggren (Chapter 35, this volume).

The Neogloboquadrina nympha Zone is denoted in the upper part of the middle Miocene by the appearance of this five-chambered marker species, that is otherwise indistinguishable from four-chambered $N$. continuosa. Neogloboquadrina nympha is probably a subspecies or even an ecophenotype of $N$. acostaensis, a species reported mainly from tropical and subtropical regions (Kennett and Srinivasan, 1983). Within the $N$. nympha Zone, a gap of about $10 \mathrm{~m}$ is present between the last appearance datum (LAD) of Globorotalia praescitula (Sample 120-747A-7H-4, 3-5 cm) and the first appearance datum (FAD) of Globorotalia scitula (Sample 120-747A-6H-4, 50-53 cm), indicating the possibility for further biostratigraphic refinement.

The Globorotalia scitula Zone is recognized in the upper Miocene on the basis of the appearance of the zonal marker. The top of this zone coincides with a hiatus level at the Miocene/Pliocene boundary.

Planktonic foraminifer faunas in the Pliocene and Pleistocene are dominated by sinistrally coiled Neogloboquadrina pachyderma. Globorotalia sphericomiozea, however, occurred immediately after the Miocene/Pliocene boundary and disappeared soon afterward in the earliest Pliocene, making possible the recognition of the $G$. sphericomiozea Zone, as proposed by Jenkins and Srinivasan (1985). Berggren (Chapter 35, this volume) found $G$. sphericomiozea from Cores 120-747A-3H through $-4 \mathrm{H}$ in the Pliocene, a level much higher than our records of this species (Fig. 1). Whether these younger morphotypes represent true $G$. sphericomiozea or phenotypes of other globorotaline lineages requires verification.

The Neogloboquadrina pachyderma Zone, in which the marker species is dominant, is identified for the Pliocene and Pleistocene. Recognition of the Globigerina bulloides and Globorotalia puncticulata subzones may help to separate the lower Pliocene from the upper sequences. The record of $G$. puncticulata in Hole $747 \mathrm{~A}$ up to a level in the Pleistocene, however, may cast doubts on this species as the late Pliocene index that it is recognized as being elsewhere (e.g., Berggren et al., 1983; Kennett and Srinivasan, 1983; Jenkins, 1985). As a substitute, we recognized a Globigerina antarctica-Tenuitellinata uvula Subzone for the Pleistocene.

\section{Hole 749B}

An attempt was made to correlate the biostratigraphy of Hole 749B to the Antarctic Paleogene (AP) zonation scheme of Stott and Kennett (1990), as does Berggren (Chapter 31, this volume). Subsequently, seven AP zones were identified that spanned the middle Eocene (Zone AP8) to upper Oligocene (AP14) (see Table 5 and Fig. 2). In addition, the occurrence of the microperforate species Praetenuitella insolita and $P$. praegemma in Core 120-749B-3H (upper AP12) is used to define the $P$. insolita Zone, originally recognized as the Globorotalia insolita Zone by Jenkins and Orr (1972).

The oldest sequences in Hole 749B contain the Acarinina bullbrooki Zone (AP8), which is correlatable to the middle Zone P10 of Blow (1979) and Berggren and Miller (1988), according to Stott and Kennett (1990). Unlike Berggren (Chapter 31, this volume), we did not recognize the Acarinina primitiva Zone (AP7) in this hole, because of the unreliable FAD of the marker species. Guembelitria triseriata and Cassigerinelloita amekiensis are abundant in the Acarinina bullbrooki Zone, and a probable phylogenetic relationship between these two species is discussed in $\mathrm{Li}$ and Radford (1991, and this volume).

The middle/upper Eocene contact was originally placed between Cores 120-749B-4H and -5H, marked by extinctions of large Acarinina such as A. primitiva (Schlich, Wise, et al., 1989). Acarinina collactea, however, has been found in Sample $120-749 \mathrm{~B}-4 \mathrm{H}-2,53-56 \mathrm{~cm}$, a level just below this boundary, according to Stott and Kennett (1990). Their view that the boundary can be defined by the LAD of $S$. linaperta (in Sample 120-749B- 3H-6, 50-53 cm, is followed here.

The occurrence of late Eocene forms such as Globigerinatheka index and Praetenuitella insolita in Sample 120-749B$3 \mathrm{H}-1,0-2 \mathrm{~cm}$, suggests that the Eocene/Oligocene boundary lies somewhere in the lower part of Core 120-749B-2H; unfortunately, this interval has not been fully recovered (Berggren, Chapter 31, this volume). Planktonic foraminifer evidence suggests that the lowermost Oligocene is missing in this hole, because the oldest Oligocene Sample 120-749B$2 \mathrm{H}-6,50-53 \mathrm{~cm}$, contains middle Oligocene microperforate species, such as Tenuitella munda, Tenuitellinata angustiumbilicata, and Tenuitellinata juvenilis (see below).

\section{TENUITELLID PLANKTONIC FORAMINIFERS}

\section{Review of Tenuitellid Genera}

The criteria used by $\mathrm{Li}$ (1987) and Brummer (1988) for microperforate generic and species differentiation are followed here. These include such morphocharacters as wall texture, chamber shape, and growth rate as well as apertural and bullate features. Stratigraphic occurrence levels also support species differentiation. Forms that possess similar wall textures, with slight differences in other morphochar- 
acters, such as a shift in apertural position and küemmerform final chamber(s), but have a similar stratigraphic record, are probably subspecies or variants of a single species. Although the presence of bullae has been considered elsewhere as of no specific value (e.g., Hemleben et al., 1989), we found it a useful, although restricted, parameter. This is because nonbullate tenuitellid forms occurred much earlier than bullate ones despite their coexistence in younger stratigraphic levels, suggesting their independent species status. If they are biologically or genetically identical, then bullae would be expected to occur at any time of their existence, and the stratigraphic ranges of the two forms would have been identical. An example is discussed below in the case of Tenuitellinata juvenilis and its bullate descendants Globigerinita boweni, G. glutinata, and G. naparimaensis.

Table 6 summarizes the characteristics of all post-Eocene microperforate genera that are considered phylogenetically closely related. A survey of the illustrations in volumes of the Initial Reports of the Deep Sea Drilling Project reveals that many microperforate species have previously been incorrectly assigned to other genera or species (see Appen$\operatorname{dix} \mathrm{A})$.

\section{Distribution of Microperforate Species in Holes 747A and 749B}

Microperforate species in these two holes are mainly representatives of Praetenuitella, Tenuitella, Tenuitellinata, and Globigerinita (Tables 2 and 3). Stratigraphically, they range from the uppermost Eocene to Pleistocene, with a maximum diversity and abundance in the middle Oligocene to lower middle Miocene (Fig. 3). No microperforate tenuitellids are found in samples older than the latest Eocene from Hole $749 \mathrm{~B}$, in contrast to the record by Premoli Silva and Boersma (1988), who reported species of Tenuitella from early to late Eocene in Atlantic regions.

\section{Praetenuitella spp.}

Both $P$. insolita and $P$. praegemma occurred only in the upper part of Core $120-749 \mathrm{~B}-3 \mathrm{H}$; they constituted about $10 \%$ and $2 \%$ of the total fauna, respectively. No significant change in their abundance was observed through their range, between Samples 120-749B-3H-2, 50-54 cm, and -3H-1, 0-2 cm (see Table 3).

\section{Tenuitella spp.}

Tenuitella gemma is rare (about $2 \%$ ) in samples from the lower to upper Oligocene (Fig. 4). The lower Oligocene hiatuses conceal the morphological transition from $T$. gemma to $T$. munda, as reported by Jenkins (1966) and Li (1987, as atypical $T$. clemenciae), because $T$. munda from the first lower Oligocene sample has already become morphologically advanced and abundant, with a proportion up to $12 \%$ in Hole $747 \mathrm{~A}$ and $25 \%$ in Hole $749 \mathrm{~B}$. Accompanied by many individuals transitional to Tenuitellinata juvenilis, $T$. munda is a common element $(10 \%-15 \%$ on average) in Oligocene and lower Miocene samples (Fig. 4).

Three small species (with a maximum test size of about 100 $\mu \mathrm{m})$ were found in the lower to middle Miocene; however, none of them became dominant. Tenuitella minutissima first occurred in the basal Miocene and subsequently gave rise to T. clemenciae in the middle lower Miocene. This was followed by the appearance of another rare species, $T$. jamesi $n . s p$. In the middle Miocene, all these forms became extinct, leaving no descendants (Fig. 4).

\section{Tenuitellinata spp.}

Apparently $T$. juvenilis is the most common species (Fig. 4). Predominant in late Oligocene $(35 \%-48 \%)$ and Miocene $(20 \%-35 \%)$, it remained common in the Pliocene $(5 \%-15 \%)$. Samples from the uppermost Oligocene and the lower Miocene generally yield some large specimens with an average size of 180-200 $\mu \mathrm{m}$ (maximum $300 \mu \mathrm{m}$ ). These large $T$. juvenilis may constitute $3 \%-5 \%$ of the total fauna in the $>150-\mu \mathrm{m}$-size fractions.

Unlike $T$. juvenilis, however, T. uvula was a minor component (about $2 \%$ ) before the late Miocene, but it subsequently became dominant $(15 \%)$ in younger sediments. It is the only microperforate species continuing into the Pleistocene in Hole 747A (Fig. 4). Intermediates between $T$. juvenilis and T. uvula were observed in almost all of the post-late Oligocene sequences.

The larger T. angustiumbilicata, with a test of 180-200 $\mu \mathrm{m}$ in diameter, became common in the latest Oligocene and the early Miocene, where it attained $20 \%-30 \%$ and $5 \%-7 \%$ in the $<150-\mu \mathrm{m}$ - and $>150-\mu \mathrm{m}$-size fractions, respectively (Fig. 4).

Apart from $T$. juvenilis, the other species dominating the lower to middle Miocene is T. pseudoedita (20\%-25\%); unlike other Tenuitellinata, however, it is always small with a maximum test size of $80-100 \mu \mathrm{m}$. A closely linked form, T. selleyi n.sp., is also recorded, with about $2 \%-5 \%$ abundance, from the upper lower Miocene to the middle Miocene (Fig. 4).

\section{Globigerinita spp.}

One of the direct descendants of Tenuitellinata juvenilis consistently found in this study is $G$. boweni. It decreased in abundance from $5 \%-10 \%$ in samples from the upper Oligocene and the lower Miocene to $2 \%-5 \%$ in younger intervals (Fig. 4). A similar case is seen in G. naparimaensis, although it did not occur until the early Miocene and only had a sparse record in post-late Miocene sediments.

Globigerinita glutinata s.s. occurred discontinuously from the basal Miocene and remained extremely rare (about 1\%) through the upper section. A moderately high proportion of this species was found in Sample 120-747A-9H-6, 50-53 cm, with $5.8 \%$ in the $<150-\mu \mathrm{m}$-size fraction.

Globigerinita praestainforthi is phylogenetically related to the $T$. angustiumbilicata-T. pseudoedita stock although, like other Globigerinita species, it possesses an umbilical bulla. It is a minor component (about $5 \%$ ) in the middle Oligocene to the lowermost Miocene (Fig. 4).

\section{Globigerinatella spp.}

No typical specimens of $G$. insueta were found, but some forms with a spherical test are regarded as Globigerinatella sp. They occurred sparsely only between Cores 120-747A$11 \mathrm{H}$ and upper $-12 \mathrm{H}$.

\section{Summary}

Microperforate species constitute up to $80 \%-90 \%$ of the total planktonic foraminifers in the $<150-\mu \mathrm{m}$-size fractions of samples from the Oligocene and Miocene (Fig. 3). Tenuitella munda was the first dominant species, but it subsequently gave way to Tenuitellinata angustiumbilicata and $T$. juvenilis, which became the most common elements in late Oligocene to earliest Miocene. Tenuitellinata juvenilis remained significant in the rest of the Miocene and Pliocene. Small-tested Tenuitella and Tenuitellinata characterized the early to middle Miocene. After the late Miocene, T. uvula became fairly abundant, and it is the only tenuitellid species that extends into the Pleistocene (Fig. 4). 
Table 2. Quantitative data of plantonic foraminifers, Hole 747A.

\begin{tabular}{|c|c|c|c|c|c|c|c|c|c|c|c|c|c|c|c|c|c|c|c|c|c|c|c|c|c|c|c|c|}
\hline Age & $\begin{array}{l}\text { Core, section } \\
\text { interval }(\mathrm{cm})\end{array}$ & & $\begin{array}{l}\text { Depth } \\
\text { (mbsf) }\end{array}$ & 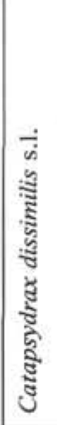 & : & 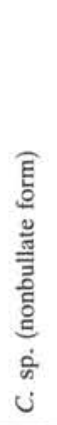 & 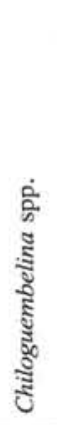 & 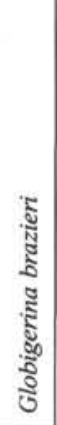 & 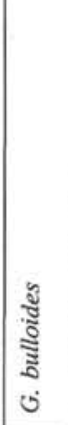 & 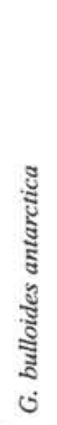 & 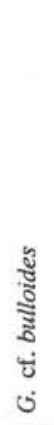 & 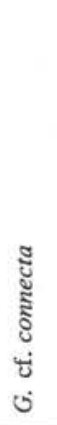 & 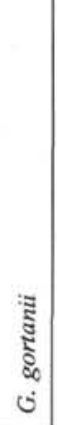 & 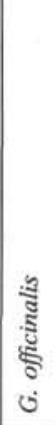 & 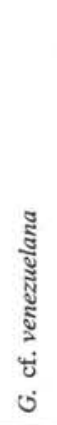 & $\begin{array}{l}\dot{i} \\
\dot{s} \\
\vdots \\
\vdots \\
\vdots \\
ن\end{array}$ & 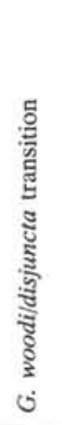 & 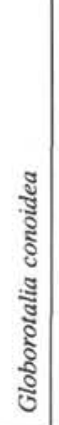 & ङัँ & $\begin{array}{l}\mathbb{N} \\
\stackrel{\Xi}{\Xi} \\
ن\end{array}$ & 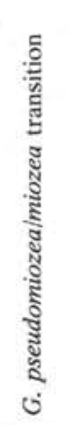 & 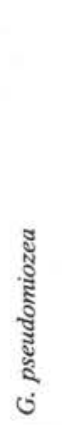 & 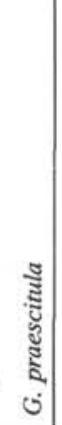 & 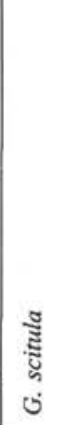 & & 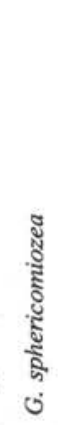 & 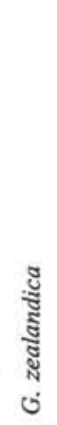 & 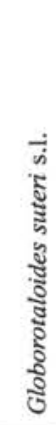 \\
\hline Pleistocene & $\begin{array}{l}1 \mathrm{H}-3,50-53 \\
1 \mathrm{H}-\mathrm{CC}, 0-2 \\
2 \mathrm{H}-3,50-53\end{array}$ & $\begin{array}{l}(\mathrm{A}) \\
(\mathrm{B}) \\
(\mathrm{A}) \\
(\mathrm{B}) \\
(\mathrm{A}) \\
(\mathrm{B}) \\
\end{array}$ & 12.5 & $\begin{array}{l}- \\
- \\
z \\
-\end{array}$ & $\begin{array}{l}- \\
- \\
- \\
-\end{array}$ & $\begin{array}{l}- \\
- \\
- \\
-\end{array}$ & $\begin{array}{l}\overline{-} \\
\bar{E} \\
\overline{-}\end{array}$ & $\begin{array}{l}- \\
- \\
- \\
- \\
-\end{array}$ & $\begin{array}{r}12 \\
7 \\
5 \\
2 \\
- \\
\end{array}$ & $\begin{array}{r}5 \\
2 \\
6 \\
-7 \\
21\end{array}$ & $\begin{array}{l}\overline{-} \\
\bar{z} \\
\overline{-}\end{array}$ & $\begin{array}{l}\bar{z} \\
\bar{z} \\
\overline{-}\end{array}$ & $\begin{array}{l}- \\
- \\
- \\
- \\
-\end{array}$ & $\begin{array}{l}- \\
- \\
- \\
- \\
-\end{array}$ & $\begin{array}{l}- \\
\overline{-} \\
\overline{-} \\
-\end{array}$ & $\begin{array}{l}- \\
\bar{z} \\
\overline{-}\end{array}$ & $\begin{array}{l}\bar{z} \\
\bar{z} \\
\bar{z}\end{array}$ & $\begin{array}{l}- \\
- \\
- \\
- \\
-\end{array}$ & $\begin{array}{l}= \\
= \\
= \\
= \\
\end{array}$ & $\begin{array}{l}- \\
- \\
- \\
- \\
-\end{array}$ & $\begin{array}{l}- \\
- \\
- \\
- \\
-\end{array}$ & $\begin{array}{l}= \\
= \\
= \\
=\end{array}$ & $\begin{array}{l}- \\
- \\
- \\
- \\
-\end{array}$ & $\begin{array}{l}\overline{4} \\
- \\
-1 \\
-\end{array}$ & $\begin{array}{r}1 \\
\frac{15}{6} \\
- \\
-\end{array}$ & $\begin{array}{l}- \\
\overline{-} \\
\overline{-}\end{array}$ & $\begin{array}{l}\overline{-} \\
\bar{z} \\
\bar{z}\end{array}$ & $\begin{array}{l}- \\
- \\
- \\
- \\
-\end{array}$ \\
\hline $\begin{array}{l}\text { late } \\
\text { Pliocene }\end{array}$ & $\begin{array}{l}2 \mathrm{H}-6,50-53 \\
2 \mathrm{H}-\mathrm{CC}, 0-2 \\
3 \mathrm{H}-2,50-53\end{array}$ & $\begin{array}{l}\text { (A) } \\
\text { (B) } \\
\text { (A) } \\
\text { (B) } \\
\text { (A) } \\
\text { (B) } \\
\end{array}$ & 18.5 & $\begin{array}{l}- \\
\overline{-} \\
- \\
-\end{array}$ & $\begin{array}{l}- \\
- \\
- \\
- \\
-\end{array}$ & $\begin{array}{l}- \\
- \\
- \\
- \\
-\end{array}$ & $\begin{array}{l}z \\
z \\
z \\
-\end{array}$ & $\begin{array}{l}- \\
- \\
- \\
- \\
-\end{array}$ & $\begin{array}{l}\frac{1}{15} \\
\frac{21}{21} \\
-\end{array}$ & $\begin{array}{l}1 \\
- \\
- \\
- \\
-\end{array}$ & $\begin{array}{l}- \\
- \\
- \\
- \\
-\end{array}$ & $\begin{array}{l}- \\
- \\
- \\
-\end{array}$ & $\begin{array}{l}- \\
- \\
- \\
- \\
-\end{array}$ & $\begin{array}{l}- \\
- \\
- \\
- \\
-\end{array}$ & $\begin{array}{l}- \\
\bar{z} \\
\bar{z} \\
\overline{-}\end{array}$ & $\begin{array}{l}- \\
- \\
- \\
-\end{array}$ & $\begin{array}{l}= \\
= \\
= \\
=\end{array}$ & $\begin{array}{l}- \\
- \\
- \\
- \\
-\end{array}$ & $\begin{array}{l}- \\
- \\
- \\
- \\
- \\
\end{array}$ & $\begin{array}{l}- \\
- \\
- \\
- \\
-\end{array}$ & $\begin{array}{l}- \\
- \\
- \\
- \\
-\end{array}$ & $\begin{array}{l}- \\
- \\
- \\
- \\
-\end{array}$ & $\begin{array}{l}- \\
- \\
- \\
- \\
-\end{array}$ & $\begin{array}{l}- \\
-1 \\
- \\
- \\
-\end{array}$ & $\begin{array}{l}\overline{-} \\
\frac{11}{1} \\
\frac{1}{-}\end{array}$ & $\begin{array}{l}\bar{z} \\
\bar{z} \\
\overline{-}\end{array}$ & $\begin{array}{l}\bar{z} \\
\bar{z} \\
\bar{z}\end{array}$ & $\begin{array}{l}\overline{-} \\
- \\
- \\
-\end{array}$ \\
\hline $\begin{array}{l}\text { early } \\
\text { Pliocene }\end{array}$ & $\begin{array}{l}3 \mathrm{H}-5,50-53 \\
3 \mathrm{H}-\mathrm{CC}, 0-3 \\
4 \mathrm{H}-2,50-53 \\
4 \mathrm{H}-3,50-52 \\
4 \mathrm{H}-4,50-53 \\
4 \mathrm{H}-5,50-52 \\
4 \mathrm{H}-6,50-53\end{array}$ & $\begin{array}{l}\text { (A) } \\
(\mathrm{B}) \\
(\mathrm{A}) \\
(\mathrm{B}) \\
(\mathrm{A}) \\
(\mathrm{B}) \\
(\mathrm{A}) \\
(\mathrm{B}) \\
(\mathrm{A}) \\
(\mathrm{B}) \\
(\mathrm{A}) \\
(\mathrm{B}) \\
(\mathrm{A}) \\
(\mathrm{B})\end{array}$ & 28.0 & $\begin{array}{l}\bar{z} \\
\overline{-} \\
\overline{-} \\
\overline{-} \\
\bar{z} \\
\bar{z} \\
\bar{z} \\
-\end{array}$ & $\begin{array}{l}\bar{z} \\
\overline{-} \\
\overline{-} \\
\bar{z} \\
\overline{-} \\
\overline{-} \\
\overline{-} \\
\overline{-}\end{array}$ & $\begin{array}{l}\bar{z} \\
\overline{-} \\
\overline{-} \\
\bar{z} \\
\overline{-} \\
\overline{-} \\
\overline{-} \\
\overline{-}\end{array}$ & $\begin{array}{l}\bar{z} \\
\bar{z} \\
- \\
- \\
\overline{-} \\
- \\
- \\
\overline{-} \\
\overline{-}\end{array}$ & $\begin{array}{l}- \\
- \\
- \\
- \\
- \\
- \\
- \\
- \\
- \\
- \\
- \\
-\end{array}$ & $\begin{array}{r}86 \\
3 \\
10 \\
\overline{75} \\
5 \\
85 \\
\overline{96} \\
\overline{52} \\
52 \\
3 \\
65 \\
14\end{array}$ & 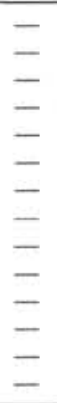 & $\begin{array}{l}- \\
\overline{-} \\
- \\
- \\
\overline{-} \\
\overline{6} \\
24 \\
3 \\
10 \\
18 \\
4 \\
-\end{array}$ & $\begin{array}{l}\bar{z} \\
\bar{z} \\
\bar{z} \\
\bar{z} \\
\bar{z} \\
\bar{z} \\
\bar{z} \\
-\end{array}$ & $\begin{array}{l}- \\
- \\
- \\
- \\
- \\
- \\
- \\
- \\
- \\
- \\
- \\
- \\
-\end{array}$ & $\begin{array}{l}- \\
- \\
- \\
- \\
- \\
- \\
- \\
- \\
- \\
- \\
-\end{array}$ & $\begin{array}{l}\bar{z} \\
\overline{-} \\
\overline{-} \\
\overline{-} \\
\overline{-} \\
\overline{-} \\
\overline{-} \\
\overline{-}\end{array}$ & $\begin{array}{l}\bar{z} \\
\bar{z} \\
\bar{z} \\
\bar{z} \\
\bar{z} \\
\bar{z} \\
\bar{z} \\
-\end{array}$ & $\begin{array}{l}\bar{z} \\
= \\
= \\
= \\
= \\
= \\
= \\
= \\
=\end{array}$ & $\begin{array}{l}- \\
- \\
- \\
- \\
- \\
- \\
- \\
- \\
- \\
- \\
- \\
- \\
-\end{array}$ & $\begin{array}{l}- \\
- \\
- \\
- \\
- \\
- \\
- \\
= \\
= \\
- \\
- \\
-\end{array}$ & $\begin{array}{l}\bar{I} \\
\bar{z} \\
\overline{-} \\
\bar{E} \\
\bar{z} \\
\overline{-} \\
\overline{-} \\
\bar{z} \\
\overline{-}\end{array}$ & 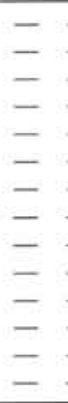 & $\begin{array}{l}- \\
z \\
- \\
- \\
= \\
= \\
= \\
= \\
= \\
-\end{array}$ & $\begin{array}{l}- \\
- \\
- \\
- \\
- \\
- \\
- \\
- \\
- \\
- \\
- \\
-\end{array}$ & \begin{tabular}{|r}
18 \\
6 \\
3 \\
2 \\
3 \\
4 \\
1 \\
$\overline{20}$ \\
15 \\
4 \\
7 \\
26 \\
\end{tabular} & $\begin{array}{l}17 \\
2 \\
- \\
\overline{7} \\
- \\
- \\
- \\
- \\
- \\
- \\
-\end{array}$ & $\begin{array}{l}- \\
- \\
- \\
- \\
- \\
- \\
- \\
- \\
\overline{-} \\
26 \\
10 \\
38 \\
5\end{array}$ & $\begin{array}{l}\overline{-} \\
- \\
- \\
- \\
= \\
- \\
\overline{-} \\
\overline{-} \\
-\end{array}$ & $\begin{array}{l}- \\
= \\
= \\
= \\
= \\
= \\
= \\
= \\
= \\
-\end{array}$ \\
\hline late & $\begin{array}{l}5 \mathrm{H}-1,50-54 \\
5 \mathrm{H}-2,50-53 \\
5 \mathrm{H}-4,50-53 \\
5 \mathrm{H}-6,50-53 \\
5 \mathrm{H}-\mathrm{CC}, 0-3 \\
6 \mathrm{H}-2,50-53 \\
6 \mathrm{H}-4,50-53\end{array}$ & $\begin{array}{l}\text { (A) } \\
(\mathrm{B}) \\
(\mathrm{A}) \\
(\mathrm{B}) \\
(\mathrm{A}) \\
(\mathrm{B}) \\
(\mathrm{A}) \\
(\mathrm{B}) \\
(\mathrm{A}) \\
(\mathrm{B}) \\
(\mathrm{A}) \\
(\mathrm{B}) \\
(\mathrm{A}) \\
(\mathrm{B}) \\
(\mathrm{A}) \\
\text { (B) }\end{array}$ & 42.5 & $\begin{array}{l}- \\
- \\
- \\
- \\
- \\
- \\
- \\
- \\
- \\
- \\
- \\
- \\
- \\
-\end{array}$ & $\begin{array}{l}\bar{z} \\
= \\
\overline{-} \\
\bar{z} \\
\bar{z} \\
\overline{-} \\
\overline{-} \\
\overline{-} \\
\overline{-} \\
\overline{-}\end{array}$ & $\begin{array}{l}\bar{z} \\
\overline{-} \\
\overline{-} \\
\bar{z} \\
\bar{z} \\
\overline{-} \\
\overline{-} \\
\bar{z} \\
\overline{-} \\
-\end{array}$ & 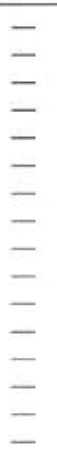 & $\begin{array}{l}- \\
- \\
- \\
- \\
- \\
- \\
- \\
- \\
- \\
- \\
- \\
- \\
- \\
- \\
-\end{array}$ & $\begin{array}{r}6 \\
8 \\
37 \\
4 \\
23 \\
7 \\
140 \\
15 \\
128 \\
3 \\
96 \\
5 \\
115 \\
10 \\
103 \\
6\end{array}$ & $\begin{array}{l}\bar{z} \\
= \\
\overline{-} \\
\bar{z} \\
\bar{z} \\
\overline{-} \\
\overline{-} \\
\bar{z} \\
\overline{-}\end{array}$ & $\begin{array}{l}\frac{5}{8} \\
\frac{5}{5} \\
\frac{17}{9} \\
\frac{9}{13} \\
\frac{21}{12} \\
-\end{array}$ & $\begin{array}{l}- \\
= \\
- \\
- \\
= \\
= \\
- \\
= \\
= \\
= \\
- \\
-\end{array}$ & $\begin{array}{l}- \\
- \\
- \\
- \\
- \\
- \\
- \\
- \\
- \\
- \\
- \\
- \\
- \\
- \\
-\end{array}$ & $\begin{array}{l}- \\
- \\
- \\
- \\
- \\
- \\
- \\
- \\
- \\
- \\
- \\
- \\
- \\
-\end{array}$ & $\begin{array}{l}- \\
\overline{-} \\
- \\
\overline{-} \\
\overline{-} \\
\overline{-} \\
\overline{-} \\
\overline{-} \\
\overline{-} \\
\overline{-} \\
-\end{array}$ & $\begin{array}{l}= \\
= \\
= \\
= \\
= \\
= \\
= \\
= \\
= \\
-\end{array}$ & $\begin{array}{l}- \\
= \\
= \\
= \\
= \\
= \\
= \\
= \\
= \\
= \\
-\end{array}$ & $\begin{array}{c}-- \\
-6 \\
2 \\
6 \\
5 \\
- \\
- \\
- \\
- \\
- \\
- \\
- \\
- \\
-\end{array}$ & $\begin{array}{l}- \\
= \\
- \\
- \\
= \\
= \\
= \\
= \\
= \\
= \\
- \\
-\end{array}$ & 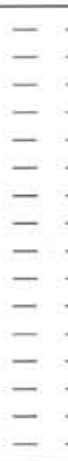 & 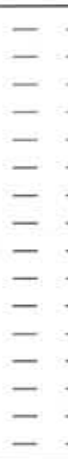 & $\begin{array}{l}- \\
= \\
= \\
= \\
= \\
= \\
= \\
= \\
= \\
= \\
= \\
-\end{array}$ & $\begin{array}{l}- \\
z- \\
- \\
z \\
z \\
z \\
- \\
- \\
- \\
- \\
- \\
-\end{array}$ & \begin{tabular}{|r}
46 \\
37 \\
34 \\
22 \\
10 \\
16 \\
15 \\
29 \\
18 \\
5 \\
22 \\
7 \\
33 \\
12 \\
28 \\
15
\end{tabular} & $\begin{array}{l}- \\
z \\
- \\
z \\
z \\
z \\
z \\
- \\
- \\
- \\
- \\
- \\
-\end{array}$ & $\begin{array}{l}\bar{z} \\
= \\
= \\
= \\
= \\
= \\
= \\
= \\
= \\
= \\
-\end{array}$ & $\begin{array}{l}\overline{-} \\
\overline{-} \\
\overline{-} \\
\bar{z} \\
\overline{-} \\
\overline{-} \\
\overline{-} \\
\bar{z} \\
\overline{-}\end{array}$ & 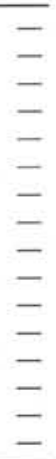 \\
\hline Miocene & $\begin{array}{l}6 \mathrm{H}-5,100-102 \\
6 \mathrm{H}-6,3-5 \\
6 \mathrm{H}-6,50-53 \\
6 \mathrm{H}-\mathrm{CC}, 0-3 \\
7 \mathrm{H}-2,50-53 \\
7 \mathrm{H}-2,99-101 \\
7 \mathrm{H}-3,50-52 \\
7 \mathrm{H}-3,97-99 \\
7 \mathrm{H}-4,3-5 \\
7 \mathrm{H}-4,52-55\end{array}$ & $\begin{array}{l}(\mathrm{A}) \\
\text { (B) } \\
(\mathrm{A}) \\
\text { (B) } \\
(\mathrm{A}) \\
(\mathrm{B}) \\
(\mathrm{A}) \\
(\mathrm{B}) \\
(\mathrm{A}) \\
(\mathrm{B}) \\
(\mathrm{A}) \\
(\mathrm{B}) \\
(\mathrm{A}) \\
(\mathrm{B}) \\
(\mathrm{A}) \\
\text { (B) } \\
\text { (A) } \\
\text { (B) } \\
\text { (A) } \\
\text { (B) }\end{array}$ & $\begin{array}{l}54.0 \\
54.5 \\
55.0\end{array}$ & $\begin{array}{l}- \\
- \\
- \\
- \\
- \\
= \\
= \\
= \\
= \\
= \\
= \\
- \\
- \\
-\end{array}$ & $\begin{array}{l}- \\
= \\
= \\
= \\
= \\
= \\
= \\
= \\
= \\
= \\
= \\
= \\
-\end{array}$ & $\begin{array}{l}= \\
= \\
= \\
= \\
= \\
= \\
= \\
= \\
= \\
= \\
= \\
=\end{array}$ & $\begin{array}{l}- \\
= \\
= \\
= \\
= \\
= \\
= \\
= \\
= \\
= \\
= \\
= \\
-\end{array}$ & $\begin{array}{l}- \\
- \\
- \\
- \\
- \\
- \\
- \\
- \\
- \\
- \\
- \\
- \\
- \\
- \\
-\end{array}$ & $\begin{array}{r}108 \\
15 \\
158 \\
23 \\
165 \\
11 \\
148 \\
5 \\
153 \\
8 \\
170 \\
12 \\
28 \\
6 \\
21 \\
7 \\
4 \\
56 \\
6\end{array}$ & $\begin{array}{l}= \\
= \\
= \\
= \\
= \\
= \\
= \\
= \\
= \\
= \\
= \\
= \\
-\end{array}$ & $\begin{array}{l}- \\
- \\
= \\
= \\
= \\
= \\
\bar{?} \\
= \\
= \\
= \\
- \\
- \\
-\end{array}$ & $\begin{array}{l}= \\
= \\
= \\
= \\
= \\
= \\
= \\
= \\
= \\
= \\
= \\
=\end{array}$ & $\begin{array}{l}- \\
- \\
- \\
- \\
= \\
- \\
- \\
- \\
- \\
- \\
- \\
= \\
= \\
- \\
- \\
-\end{array}$ & $\begin{array}{l}- \\
- \\
- \\
- \\
- \\
- \\
- \\
- \\
- \\
- \\
- \\
- \\
- \\
- \\
- \\
- \\
-\end{array}$ & $\begin{array}{l}\overline{-} \\
\overline{-} \\
\overline{-} \\
\bar{z} \\
\bar{z} \\
\overline{-} \\
\overline{-} \\
\overline{-} \\
\overline{-} \\
\overline{-} \\
\overline{-} \\
-\end{array}$ & $\begin{array}{l}= \\
= \\
= \\
= \\
= \\
= \\
= \\
= \\
= \\
= \\
= \\
=\end{array}$ & $\begin{array}{l}= \\
= \\
= \\
= \\
= \\
= \\
= \\
= \\
= \\
= \\
= \\
= \\
=\end{array}$ & $\begin{array}{l}- \\
- \\
- \\
- \\
- \\
- \\
- \\
- \\
- \\
- \\
- \\
- \\
- \\
- \\
- \\
- \\
- \\
-\end{array}$ & $\begin{array}{l}= \\
= \\
= \\
= \\
= \\
= \\
= \\
= \\
= \\
= \\
= \\
= \\
= \\
= \\
=\end{array}$ & 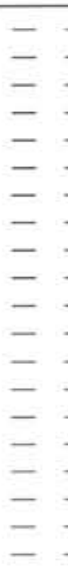 & 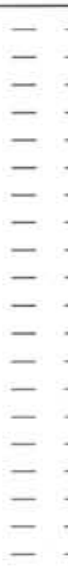 & $\begin{array}{l}= \\
= \\
= \\
= \\
= \\
= \\
= \\
= \\
= \\
= \\
= \\
= \\
= \\
= \\
= \\
=\end{array}$ & $\begin{array}{l}- \\
z- \\
z- \\
- \\
- \\
- \\
- \\
- \\
- \\
- \\
- \\
- \\
- \\
- \\
- \\
7 \\
11 \\
35 \\
10\end{array}$ & $\begin{array}{l}? \\
- \\
- \\
- \\
- \\
- \\
= \\
- \\
- \\
- \\
- \\
- \\
- \\
- \\
- \\
- \\
-\end{array}$ & $\begin{array}{l}- \\
= \\
= \\
= \\
= \\
= \\
= \\
= \\
= \\
= \\
= \\
= \\
= \\
= \\
=\end{array}$ & $\begin{array}{l}- \\
= \\
= \\
= \\
= \\
= \\
= \\
= \\
= \\
= \\
= \\
= \\
= \\
-\end{array}$ & 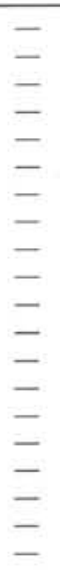 & $\begin{array}{l}- \\
- \\
- \\
- \\
- \\
- \\
- \\
- \\
- \\
- \\
- \\
- \\
- \\
- \\
- \\
- \\
- \\
-\end{array}$ \\
\hline
\end{tabular}


Table 2 (continued).

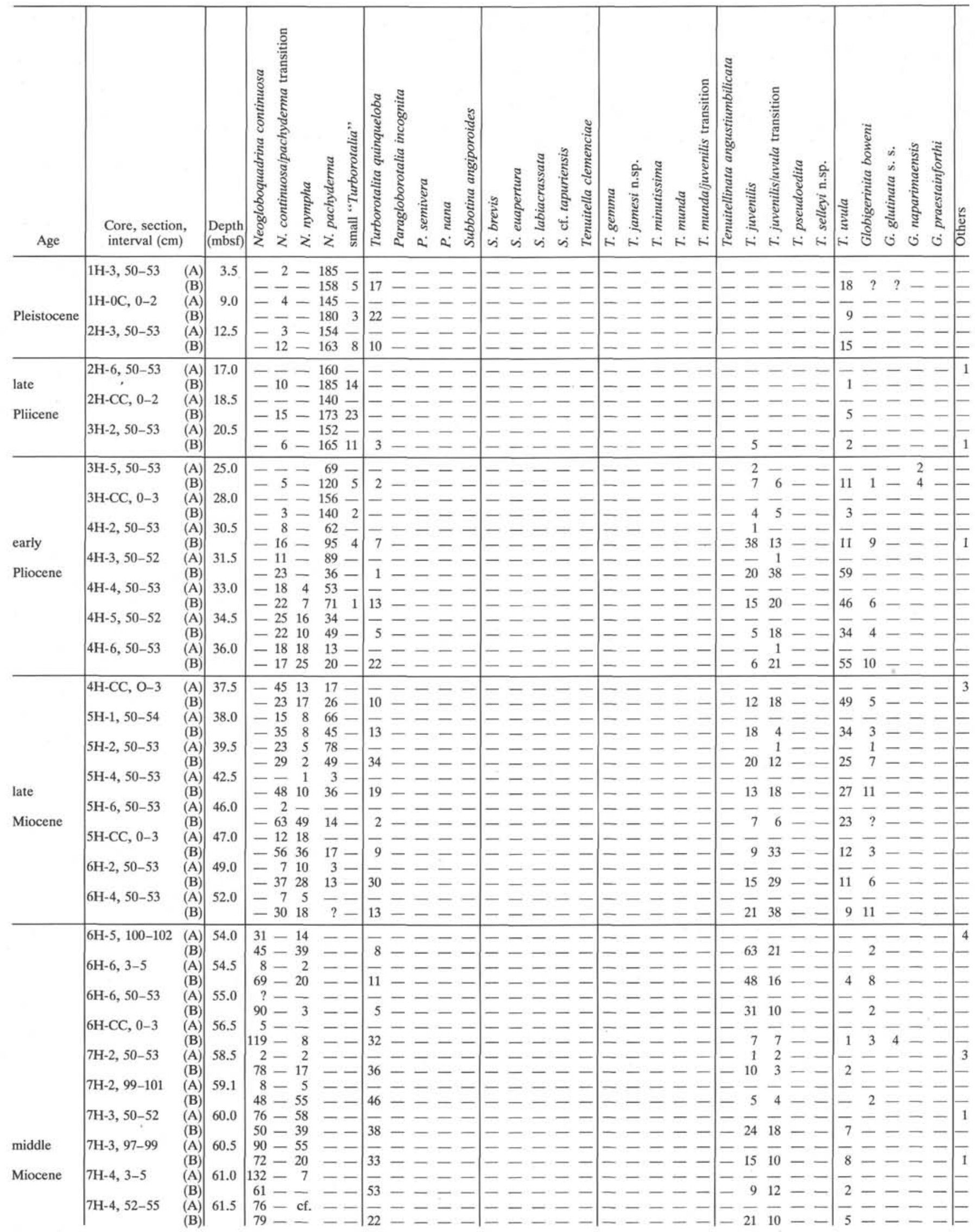


Table 2 (continued).

\begin{tabular}{|c|c|c|c|c|c|c|c|c|c|c|c|c|c|c|c|c|c|c|c|c|c|c|c|c|c|c|c|c|}
\hline \multirow[t]{20}{*}{ Age } & \multicolumn{2}{|l|}{$\begin{array}{l}\text { Core, section, } \\
\text { interval }(\mathrm{cm})\end{array}$} & \multirow{2}{*}{\begin{tabular}{|c}
$\begin{array}{c}\text { Depth } \\
\text { (mbsf) }\end{array}$ \\
62.0
\end{tabular}} & 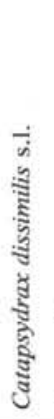 & 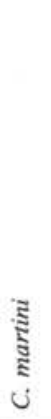 & 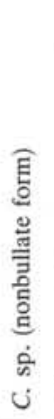 & 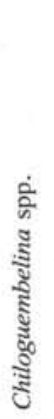 & 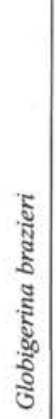 & 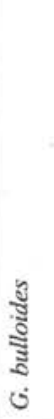 & 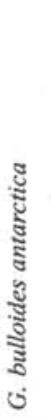 & $\begin{array}{l}\hat{y} \\
\tilde{\Xi} \\
\bar{\Xi} \\
0 \\
\dot{U} \\
ن\end{array}$ & 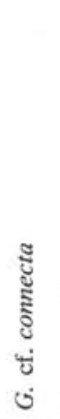 & 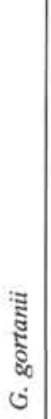 & 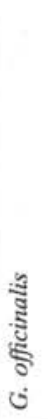 & 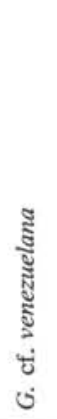 & $\begin{array}{c} \\
\\
\dot{\vdots} \\
\dot{\sim} \\
\dot{\Xi} \\
\vdots \\
\vdots \\
\vdots\end{array}$ & 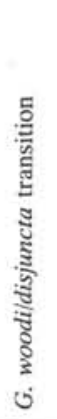 & 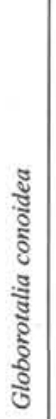 & $\begin{array}{l}\text { हैँ } \\
\text { ลे } \\
ن\end{array}$ & 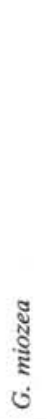 & 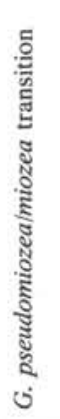 & 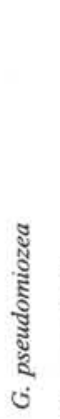 & 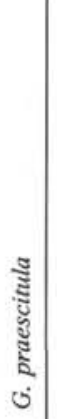 & 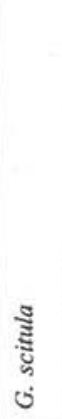 & 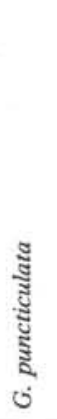 & 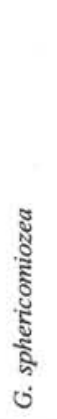 & 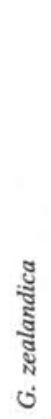 & 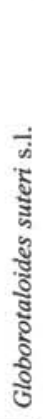 \\
\hline & $7 \mathrm{H}-4,98-100$ & (A) & & - & - & - & - & - & 45 & - & - & - & -1 & - & - & - & - & -1 & - & - & - & - & 89 & - & - & - & - & - \\
\hline & & (B) & & - & - & - & - & - & 20 & - & - & - & - & - & - & - & - & - & - & - & - & - & 28 & - & - & - & - & - \\
\hline & $7 \mathrm{H}-5,52-54$ & (A) & 63.0 & - & - & - & - & - & 38 & - & - & - & -1 & - & - & - & - & - & - & - & - & -1 & 103 & - & - & - & - & - \\
\hline & & (B) & & - & - & - & - & - & 20 & - & - & - & - & - & - & - & - & - & - & - & - & - & 43 & - & - & - & - & - \\
\hline & $7 \mathrm{H}-6,50-53$ & (A) & 64.5 & - & - & - & - & - & 21 & - & - & - & - & - & - & - & - & - & - & - & - & -1 & 118 & - & - & - & - & - \\
\hline & $7 \mathrm{H}-\mathrm{CC}, 0-3$ & (B) & & - & - & - & - & - & 14 & - & - & - & - & - & - & - & - & - & - & - & - & - & 20 & - & - & - & - & - \\
\hline & & $\begin{array}{l}\text { (A) } \\
\text { (B) }\end{array}$ & 66.0 & I & I & - & - & - & $\begin{array}{l}42 \\
22\end{array}$ & 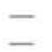 & - & cf. & 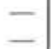 & - & - & I & I & - & $\overline{-}$ & $\overline{-}$ & $\overline{-}$ & - & $\begin{array}{l}68 \\
31\end{array}$ & $\overline{-}$ & - & 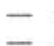 & $\overline{-}$ & - \\
\hline & $8 \mathrm{H}-2,55-58$ & (A) & 68.1 & - & - & - & - & - & 96 & - & - & 2 & -1 & - & - & - & - & - & - & $\overline{6}$ & - & - & $\begin{array}{l}31 \\
11\end{array}$ & $\overline{-}$ & - & $=$ & $=$ & - \\
\hline & & (B) & & - & - & - & - & -1 & 16 & - & - & - & -1 & - & - & - & - & - & - & - & - & - & - & - & - & - & - & - \\
\hline & $8 \mathrm{H}-4,55-58$ & (A) & 71.0 & - & - & - & - & - & 40 & - & - & 15 & - & - & - & 28 & 10 & - & 28 & 21 & - & - & 30 & - & - & - & - & - \\
\hline & & (B) & & - & - & - & - & -1 & 11 & - & - & - & - & - & - & 8 & - & - & - & - & - & 9 & 26 & - & - & - & - & - \\
\hline & $8 \mathrm{H}-6,55-58$ & (A) & 74.1 & - & - & - & - & - & 4 & - & - & 10 & - & - & - & 25 & 15 & - & 16 & 63 & - & - & 19 & - & - & - & - & - \\
\hline & & (B) & & - & - & - & - & - & 12 & - & - & - & - & - & - & 3 & - & - & - & - & - & 2 & 48 & - & - & - & - & - \\
\hline & $8 \mathrm{H}-\mathrm{CC}, 0-3$ & (A) & 75.5 & - & - & - & - & - & 5 & - & - & 25 & - & - & - & 40 & 16 & - & $?$ & 11 & - & 5 & 63 & - & - & - & - & - \\
\hline & & (B) & & - & - & - & - & - & 8 & - & - & 2 & - & - & - & 4 & - & - & - & - & 8 & 3 & 32 & - & - & - & - & - \\
\hline & $9 \mathrm{H}-2,50-53$ & (A) & 76.0 & - & - & - & - & - & 4 & - & - & 15 & - & - & - & 13 & 4 & - & - & 58 & 21 & 26 & 39 & - & - & - & - & - \\
\hline & & (B) & & - & - & - & - & - & 4 & - & - & - & - & - & - & 3 & - & - & - & - & 6 & - & 20 & - & - & - & - & - \\
\hline & $9 \mathrm{H}-4,50-53$ & (A) & 79.0 & - & - & - & - & - & 3 & - & - & 10 & - & - & - & 18 & 3 & - & - & 44 & 38 & 35 & 22 & - & - & - & cf. & - \\
\hline & & (B) & & - & - & - & - & - & 2 & - & - & - & - & - & - & 5 & - & - & - & - & - & - & 26 & - & - & - & - & - \\
\hline & $9 \mathrm{H}-5,50-53$ & (A) & 80.5 & - & - & - & - & - & 17 & - & - & 11 & - & - & - & 35 & 6 & - & - & - & 10 & 53 & 3 & - & - & - & 15 & - \\
\hline early & & (B) & & - & - & - & - & -1 & 5 & - & - & - & - & - & - & 2 & - & - & - & - & - & - & 18 & - & - & - & 12 & - \\
\hline & $9 \mathrm{H}-6,50-53$ & (A) & 82.0 & - & - & - & - & - & 3 & - & - & 7 & - & - & - & 31 & 2 & - & - & - & 16 & 45 & 6 & - & - & - & 21 & - \\
\hline & & (B) & & - & - & - & - & -1 & 12 & - & - & 1 & - & - & - & 5 & - & - & - & - & - & 13 & 12 & - & - & - & 23 & - \\
\hline & $9 \mathrm{H}-6,104-106$ & (A) & 82.7 & - & - & - & - & - & 2 & - & - & 4 & - & - & - & 21 & 5 & - & - & - & 7 & 78 & 18 & - & - & - & 14 & - \\
\hline & & (B) & & - & - & - & - & - & - & - & - & - & - & - & - & 7 & - & - & - & - & - & 9 & 27 & - & - & - & 13 & - \\
\hline & $9 \mathrm{H}-7,0-2$ & (A) & 83.0 & - & - & - & - & - & 8 & - & - & - & - & - & - & 5 & - & - & - & - & - & 108 & 22 & - & - & - & 21 & - \\
\hline & & (B) & & - & - & - & - & - & 11 & - & - & - & - & - & - & 8 & - & - & - & - & - & - & 20 & - & - & - & - & - \\
\hline & $9 \mathrm{H}-7,50-52$ & (A) & 83.5 & - & - & - & - & - & 38 & - & - & - & - & - & - & - & - & - & - & - & - & 113 & 15 & - & - & - & 10 & - \\
\hline & & (B) & & - & - & - & - & - & 9 & - & - & - & - & - & - & 6 & - & - & - & - & - & 13 & 45 & - & - & - & 15 & - \\
\hline & $9 \mathrm{H}-7,104-106$ & (A) & 84.0 & - & - & - & - & - & 36 & - & - & 11 & - & - & - & 1 & 1 & - & - & - & - & 100 & 29 & - & - & - & 26 & - \\
\hline & & (B) & & - & - & - & - & - & 12 & - & - & - & - & - & - & - & 4 & - & - & - & - & 17 & 62 & - & - & - & - & - \\
\hline & $9 \mathrm{H}-\mathrm{CC}, 0-3$ & (A) & 85.0 & - & - & - & - & - & 6 & - & - & cf. & - & - & - & 4 & - & - & - & - & - & 42 & 89 & - & - & - & 4 & - \\
\hline & & (B) & & - & - & - & - & - & 3 & - & - & - & - & - & - & 3 & - & - & - & - & - & 38 & 26 & - & - & - & - & - \\
\hline & $10 \mathrm{H}-1,53-55$ & (A) & 85.6 & - & - & - & - & - & 19 & - & - & - & - & - & - & 38 & - & -1 & - & - & - & 29 & 68 & - & - & - & 3 & 2 \\
\hline & & (B) & & - & - & - & - & - & 5 & - & - & - & - & - & - & 24 & - & - & - & - & - & 8 & 17 & - & - & - & - & 2 \\
\hline & $10 \mathrm{H}-1,103-105$ & (A) & 86.0 & - & - & - & - & - & 35 & - & - & - & - & - & - & 38 & - & - & - & - & - & 33 & 48 & - & - & - & 7 & - \\
\hline & & (B) & & - & - & - & - & - & $?$ & - & - & - & - & - & - & 20 & - & - & - & - & - & 15 & 12 & - & - & - & - & 3 \\
\hline & $10 \mathrm{H}-2,0-3$ & $\begin{array}{l}\text { (A) } \\
\text { (B) }\end{array}$ & 86.5 & $\bar{z}$ & - & $\overline{-}$ & - & - & $\begin{array}{l}7 \\
8\end{array}$ & - & - & - & - & - & - & 5 & - & - & - & - & - & 125 & 22 & - & - & - & $?$ & - \\
\hline & $10 \mathrm{H}-2,50-53$ & (A) & 87.0 & 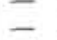 & - & - & $\bar{z}$ & - & $\begin{array}{r}8 \\
66\end{array}$ & - & $\overline{-}$ & - & - & - & $\bar{z}$ & $\overline{c f}$. & $\overline{-}$ & - & $\bar{z}$ & - & $\overline{-}$ & $\begin{array}{l}17 \\
53\end{array}$ & 10 & $z$ & - & - & $\overline{9}$ & 5 \\
\hline & & (B) & & - & - & - & - & - & 3 & - & - & - & - & - & - & 4 & - & - & - & - & - & $\begin{array}{r}53 \\
8\end{array}$ & $\begin{array}{r}20 \\
7\end{array}$ & $z$ & - & - & - & $\overline{2}$ \\
\hline & $10 \mathrm{H}-4,52-55$ & (A) & 90.0 & - & - & - & - & - & 93 & - & - & - & -1 & - & - & 2 & - & - & - & - & - & 38 & 15 & - & - & - & 4 & 2 \\
\hline early & & (B) & & - & - & - & - & - & 21 & - & - & - & - & - & - & 12 & - & - & - & - & - & 5 & 18 & - & - & - & - & 6 \\
\hline & $10 \mathrm{H}-6,52-55$ & (A) & 93.0 & - & - & 18 & - & - & 20 & - & - & - & - & - & - & 19 & - & - & - & - & - & 78 & 21 & - & - & - & 8 & 3 \\
\hline Miocene & $\mathrm{H}-\mathrm{CC}, 0-3$ & (D) & 94.5 & - & - & $\overline{10}$ & - & - & 6 & - & - & - & -1 & - & - & 22 & - & - & - & - & - & 34 & 23 & - & - & - & - & 10 \\
\hline & & $\begin{array}{l}\text { (A) } \\
\text { (B) }\end{array}$ & 94.5 & - & - & 10 & - & - & $\begin{array}{l}28 \\
15\end{array}$ & - & - & - & - & - & - & 10 & - & - & - & - & - & 12 & 67 & - & - & - & 3 & 7 \\
\hline & $11 \mathrm{H}-2,50-53$ & (A) & 96.5 & - & - & 11 & $\overline{-}$ & - & $\begin{array}{l}13 \\
10\end{array}$ & - & - & - & - & - & - & $\begin{array}{l}41 \\
80\end{array}$ & - & $\bar{z}$ & $\bar{z}$ & - & $\overline{-}$ & 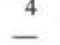 & $\begin{array}{l}16 \\
20\end{array}$ & $=$ & - & - & $\bar{z}$ & $\begin{array}{r}12 \\
5\end{array}$ \\
\hline & & (B) & & - & - & - & - & - & - & - & - & - & - & - & - & 55 & - & - & - & - & - & - & 12 & - & - & - & $\overline{-}$ & 9 \\
\hline & $11 \mathrm{H}-4,50-53$ & (A) & 99.5 & - & - & 3 & - & - & 8 & - & - & - & - & - & - & 54 & - & - & - & - & - & - & 23 & - & - & - & - & 3 \\
\hline & & (B) & & - & - & - & - & - & 2 & - & - & - & - & - & - & 39 & - & - & - & - & - & - & 9 & - & - & - & - & 7 \\
\hline & $11 \mathrm{H}-6,50-53$ & (A) & 102.5 & 18 & - & 5 & - & - & 12 & - & - & - & - & - & - & 38 & - & - & - & - & - & - & 12 & - & - & - & - & 4 \\
\hline & & (B) & & 11 & - & 5 & - & - & 5 & - & - & - & - & - & - & 35 & - & - & - & - & - & - & 3 & - & - & - & - & 2 \\
\hline & $11 \mathrm{H}-\mathrm{CC}, 0-3$ & (A) & 104.0 & 21 & - & 1 & - & - & 11 & - & - & - & - & - & - & 25 & - & - & - & - & - & - & 5 & - & - & - & - & - \\
\hline & & (B) & & 3 & - & - & - & - & 5 & - & - & - & - & - & - & 33 & - & - & - & - & - & - & 4 & - & - & - & - & 7 \\
\hline & $12 \mathrm{H}-2,50-53$ & (A) & 106.5 & 17 & - & 8 & - & - & 10 & - & - & - & - & - & - & 47 & - & - & - & - & - & - & - & - & - & - & - & 3 \\
\hline & & (B) & & - & - & - & - & - & - & - & - & - & - & - & - & 63 & - & - & - & - & - & - & - & - & - & - & - & 11 \\
\hline & $12 \mathrm{H}-4,50-53$ & (A) & 109.0 & 38 & - & 9 & - & - & 50 & - & - & - & - & - & - & 17 & - & -1 & - & - & - & - & - & - & - & - & - & 5 \\
\hline & & (B) & & 15 & - & - & - & - & 8 & - & - & - & - & - & - & 55 & - & -1 & - & - & - & - & - & - & - & - & - & 2 \\
\hline & $12 \mathrm{H}-6,53-56$ & (A) & 112.0 & 48 & - & 15 & - & - & 5 & - & - & - & -1 & - & - & 52 & - & -1 & - & - & - & - & - & - & - & - & - & 6 \\
\hline & & (B) & & 3 & - & - & - & -1 & 2 & - & - & - & -1 & - & - & 55 & - & - & - & - & - & - & - & - & - & - & - & 3 \\
\hline & $12 \mathrm{H}-\mathrm{CC}, 0-3$ & (A) & 113.5 & 7 & - & 3 & - & - & 23 & - & - & - & - & - & - & 95 & - & - & - & - & - & - & - & - & - & - & - & - \\
\hline & & (B) & & - & - & - & - & - & - & - & - & - & - & - & - & 58 & - & - & - & - & - & - & - & - & - & - & - & 4 \\
\hline & $13 \mathrm{H}-2,55-58$ & (A) 1 & $115.5 \mid$ & 6 & - & $?$ & - & -1 & 127 & - & - & - & -1 & - & - & 7 & - & -1 & - & - & - & - & -1 & - & - & - & - & - \\
\hline
\end{tabular}


Table 2 (continued).

\begin{tabular}{|c|c|c|c|c|c|c|c|c|c|c|c|c|c|c|c|c|c|c|c|c|c|c|c|c|c|c|c|c|c|c|c|c|}
\hline \multirow[t]{19}{*}{ Age } & \multicolumn{2}{|c|}{$\begin{array}{l}\text { Core, section, } \\
\text { interval }(\mathrm{cm})\end{array}$} & $\begin{array}{l}\text { Depth } \\
\text { (mbsf) }\end{array}$ & 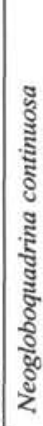 & 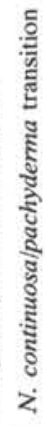 & है & $\begin{array}{l}\text { हूँ } \\
\text { हूँ } \\
\text { हूँ } \\
\text { z } \\
亡\end{array}$ & 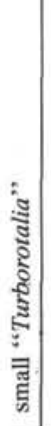 & 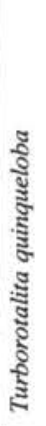 & 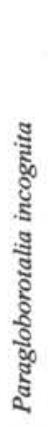 & 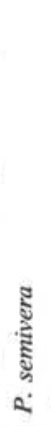 & 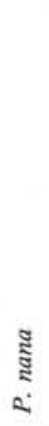 & 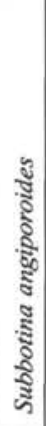 & 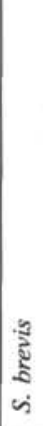 & 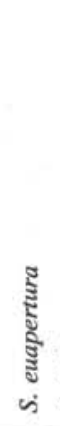 & 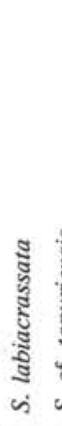 & 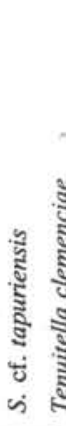 & 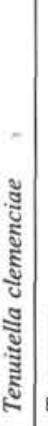 & & 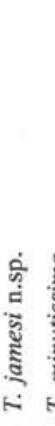 & & 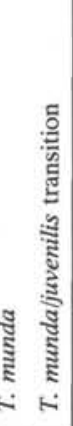 & 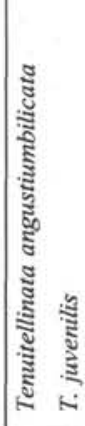 & 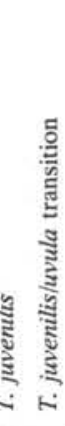 & 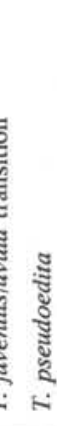 & 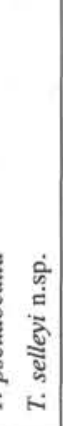 & ( & 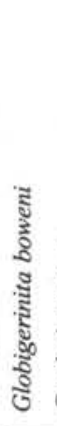 & 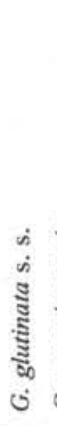 & 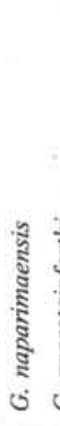 & 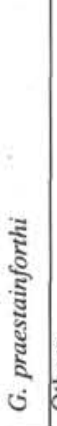 & \\
\hline & - $-4,98-100$ & & 62 & 5 & - & - & - & - & - & - & - & - & -1 & $1-$ & - & -- & - & -1 & - - & -- & - & - & & 211 & 1 - & -1 & & - & - & & - & \\
\hline & & & & 62 & - & - & - & -1 & 18 & - & - & - & - & - & - & -- & - & $-1-$ & - & -- & -- & -- & -40 & $40 \quad 23$ & - & -- & & 4 & - & - & - & \\
\hline & $7 \mathrm{H}-5,52-54$ & $(A$ & 63.0 & 21 & - & - & - & -1 & 10 & - & - & - & - & - & - & -- & - & - & -- & -- & -- & -- & -- & -1 & - & -- & - & - & - & - & - & \\
\hline & & (I & & 56 & - & - & - & -3 & 37 & - & - & - & - & - & - & - & - & - & -- & -- & -- & -- & -18 & 18 & - & -- & 4 & 2 & & - & - & \\
\hline & 1-6, & $\begin{array}{l}(f \\
(\mathrm{H}\end{array}$ & 64.5 & $\begin{array}{l}10 \\
63\end{array}$ & $\overline{-}$ & E & $\bar{z}$ & 二 & $\overline{28}$. & I & $\bar{z}$ & $\bar{z}$ & - & - & $\bar{z}$ & $\bar{z}$ & $=$ & - & $\overline{-}$ & - & $E$ & $-二$ & - & $\overline{16}$ & - & $=-$ & $\overline{5}$ & $\overline{5}$ & $\overline{-}$ & - & $=$ & \\
\hline & $7 \mathrm{H}-\mathrm{CC} \quad 0-3$ & (A) & 66.0 & 43 & - & 22 & - & - & - & $=$ & $\overline{-}$ & - & - & - & $=$ & - & -1 & $=$ & 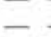 & - - & - & -- & - - & - & - & -- & - & - & - & - & - & \\
\hline & & (B) & & 55 & - & - & - & -1 & 11 & - & - & - & - & - & - & - & - & 7. & - & - & 2 & --1 & -24 & $24 \quad 20$ & 20 & $0-$ & 9 & 3 & - & 12 & - & \\
\hline & $8 \mathrm{H}-2,53-58$ & (A) & 68.1 & 38 & - & - & - & - & 2 & - & - & - & - & - & - & - & - & $-1-$ & - & - & - & --1 & -- & -- & - & -- & - & - & - & - & - & \\
\hline & & (B) & & 32 & - & - & - & -2 & 20 & - & - & - & - & - & - & - & - & 5 & - & 7 & $6-$ & --1 & -25 & 25 & 60 & $\begin{array}{ll}0 & 14\end{array}$ & 2 & - & - & - & - & \\
\hline & $8 \mathrm{H}-4,55-58$ & (A) & 71.0 & - & - & - & - & - & - & - & - & - & - & - & - & - & - & $-1-$ & - & - & -- & -- & -1 & 1 & $\overline{-1}$ & -- & - & - & - & - & - & \\
\hline & & (F & & cf. & - & - & - & -1 & 13 & - & - & - & - & - & - & - & - & 3 . & - & 4 & ? - & -- & -21 & 21 & 67 & $\begin{array}{ll}7 & 9\end{array}$ & - & - & & 8 & - & \\
\hline & $8 \mathrm{H}-6,55-58$ & (A) & 74.1 & - & - & - & - & - & $\bar{c}$ & - & - & - & - & - & - & - & - & $-1-$ & - & - & -- & -- & -7 & $\begin{array}{ll}7 & 3 \\
0\end{array}$ & - & - & $=$ & - & $\overline{0}$ & 3 & - & \\
\hline & & (B) & & - & - & - & - & -1 & 16 & - & - & - & - & - & - & - & - & 6 - & - & 8 & $4-$ & -- & -49 & 4920 & 58 & 5 & 5 & - & 3 & 11 & - & \\
\hline & 3 & (A) & 75.5 & - & - & - & - & - & $\overline{10}$ & - & - & - & - & - & - & - & - & $-1-$ & - & - & - & --1 & -5 & $5 \quad 3$ & $\overline{3 e}$ & - & - & - & $\overline{0}$ & - & - & \\
\hline & $-2,50-53$ & & & - & - & - & - & -1 & 10 & - & - & - & - & - & - & - & - & 1 & - & 2 & $1-$ & --1 & -61 & $\begin{array}{ll}51 & 10\end{array}$ & 35 & $?$ & - & 2 & 2 & 4 & - & \\
\hline & & (B) & 10.0 & 二 & $=$ & $=$ & $\bar{z}$ & - & $\overline{5}$ & $\bar{z}$ & $\overline{-}$ & - & - & - & - & - & - & -5 & - & $\overline{7}$ & ? & -- & $\begin{array}{l}-11 \\
-\quad 58\end{array}$ & $11 \overline{12}$ & $\overline{43}$ & 7 & $\overline{3}$ & $\overline{5}$ & - & $\overline{0}$ & - & \\
\hline & 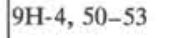 & (A) & 79.0 & - & - & - & - & -1 & - & - & - & - & - & - & - & - & - & -1 & - & - & $\div-$ & $-二$ & - 5 & $5-$ & -1 & -1 & - & - & $=$ & 5 & - & \\
\hline & & (B) & & - & - & - & - & - & 7. & - & - & - & - & - & - & - & - & 5. & & 21 & $?-$ & -- & -46 & $\begin{array}{ll}16 \quad 7 \\
\end{array}$ & 39 & $9-$ & - & 10 & 3 & & - & \\
\hline & & (A) & 0.5 & - & - & - & - & -1. & - & - & - & - & - & - & - & - & -- & $-1-$ & - & - & - & --1 & - & $9-$ & - & -- & - & - & - & - & - & \\
\hline & & (B) & & - & - & - & - & -1 & 14 & - & - & - & - & - & - & - & -14 & 14 - & - & 4 & $?$ & --1 & -41 & $41 \quad 15$ & 52 & $2-$ & - & 7 & 8 & 7 & - & \\
\hline & $9 \mathrm{H}-6,50-53$ & (A) & 82.0 & - & - & - & - & -1 & - & - & - & - & - & - & - & - & -- & - & - & - & - & -- & -15 & $15-$ & - & -- & - & - & $\bar{u}$ & $\overline{1}$ & - & \\
\hline & & (B) & & - & - & - & - & -1 & ? & - & - & - & - & - & - & - & - & 7 - - & - & 2 & $8-$ & -- & -35 & $\begin{array}{ll}35 & 10\end{array}$ & 40 & $0-$ & 2 & 7 & 11 & 12 & - & \\
\hline & $9 H-6,104-106$ & (A) & 82.7 & - & - & - & - & -1 & - & - & - & - & - & - & - & - & -- & $-1-$ & - & - - & - & -- & -- & -- & $=$ & -- & - & - & - & - & - & \\
\hline & & $\begin{array}{l}\text { (B) } \\
\text { (A) }\end{array}$ & & - & $\bar{z}$ & $\bar{z}$ & $\overline{-}$ & - & 6 & - & $\overline{7}$ & - & - & - & - & - & -2 & $22-$ & - & ? 1 & $16=$ & -- & $\begin{array}{r}-63 \\
-2\end{array}$ & $\begin{array}{ll}3 & 14 \\
2\end{array}$ & 48 & $8-$ & - & 8 & & 8 & - & \\
\hline & & (B) & 0.0 & - & $=$ & $\bar{E}$ & $=$ & $=$ & 5 & - & - & $=$ & - & - & E. & $=$ & $\overline{-1}$ & $\overline{11}$. & $\overline{-}$ & $\overline{3}$ & $?$ & -- & - 58 & $8 \overline{21}$ & 36 & $\overline{7}$ & $\overline{2}$ & $\overline{12}$ & - & $\overline{6}$ & $=$ & \\
\hline & -52 & (A) & 83.5 & - & - & - & - & $-1-$ & - & - & ? & - & - & - & - & - & -- & $-1-$ & - & - - & - & -- & -3 & $3-$ & - & - & - & - & - & - & - & \\
\hline & & (B) & & - & - & - & - & - & 7. & - & - & - & - & - & - & -- & $-18-1$ & 18 - & - & $?$ & $3-$ & -- & -42 & 8 & 30 & 13 & - & 20 & - & 9 & - & \\
\hline & $104-106$ & (A) & 84.0 & - & - & - & - & -1 & - & - & - & - & - & - & - & -- & -- & $-1-$ & - & -- & -- & -- & -4 & - & $\overline{0}$ & - & - & - & & - & - & \\
\hline & & (B) & & - & - & - & - & - & 9 & - & - & - & - & - & - & - - & -1 & $13-$ & - & $?$ & $5-$ & --1 & -35 & 4 & +25 & 5 & - & - & - & - & - & \\
\hline & $1-c$ & $\begin{array}{l}\text { (A) } \\
\text { (B) }\end{array}$ & 85.0 & - & - & - & $\overline{-}$ & - & $\overline{11}$ & $\bar{z}$ & $\overline{-}$ & - & - & - & - & - & $-\overline{3}$ & $-1-$ & - & $\overline{3}$ & 7 & -- & -6 & $\overline{6}$ & 7 & $\overline{0}-1$ & - & $\overline{2}$ & $\overline{2}$ & $\overline{0}$ & - & \\
\hline & -55 & (A) & 85.6 & - & - & - & - & $=$ & 11 & cf. & - & - & - & - & - & $=$ & $=2$ & 20 & - & 2 & $-1-$ & $-z$ & $\begin{array}{l}-31 \\
-\quad 9\end{array}$ & - & 19 & 2 & - & - & 2 & - & - & \\
\hline & & (B) & & - & - & - & - & - & 6 & - & - & - & - & - & - & -- & -- & $-1-$ & - & 71 & $14-$ & -- & -46 & $\begin{array}{ll}6 & 12\end{array}$ & 54 & 3 & 1 & 6 & - & 13 & - & \\
\hline & $10 \mathrm{H}-1,103-105$ & (A) & 86.0 & - & - & - & - & - & $\overline{-}$ & - & - & - & - & - & - & -- & -- & $-1-$ & - & - & -- & -- & -15 & $5-$ & 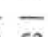 & - & - & - & - & - & - & \\
\hline & & & & - & - & - & - & -1 & 13 & - & - & - & -1 & - & - & -- & -- & -1. & - & 51 & $16-$ & --1 & -40 & $\begin{array}{ll}0 & 9\end{array}$ & 53 & ? & 3 & - & & 8 & - & \\
\hline & $10 \mathrm{H}-2,0-3$ & (A) & 86.5 & - & - & - & - & -1 & - & - & - & - & - & - & - & -- & -- & $-1-$ & - & -- & -- & --1 & -10 & - & - & - & - & - & - & - & - & \\
\hline & & & & - & - & - & - & -1 & $?$ & - & - & - & - & - & - & -- & -- & -1. & - & 32 & $21-$ & --1 & -37 & 715 & 62 & & 3 & - & 4 & 11 & - & \\
\hline & $10 \mathrm{H}-2,50-53$ & (A) & 87.0 & - & - & - & - & -1 & - & - & - & - & - & - & - & -- & -- & -1 & - & - & - & --1 & -4 & $4-$ & - & -- & - & - & - & - & - & \\
\hline & & & & - & - & - & - & -1 & 10 & - & - & - & - & - & - & -- & -11 & 11 - & - & 21 & $10-$ & -- & -39 & 920 & 53 & $\begin{array}{ll}3 & 10\end{array}$ & 9 & - & & 9 & & \\
\hline & $2-55$ & (A) & 90.0 & - & - & - & - & $-1-$ & - & - & - & - & - & - & - & -- & -- & -1. & - & - - & - & --1 & -5 & $5-$ & -- & & 3 & - & - & - & 一 & \\
\hline arly & & (B) & & - & - & - & - & & cf. & 5 & - & - & - & - & - & - & - & $?$ & - & -1 & $13-$ & --1 & -46 & 623 & 355 & $\begin{array}{ll}5 & 17\end{array}$ & 5 & 8 & & 7 & - & \\
\hline & $10 \mathrm{H}-6$ & (A) & 93.0 & - & - & - & - & $-1-$ & - & & - & - & - & - & - & - & -- & -1. & - & -- & - & -- & -4 & $4-$ & -- & & 1 & - & 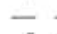 & - & - & \\
\hline & & (B) & & - & - & - & - & & - & & - & - & - & - & - & - & - & $-1-$ & - & ? & $7-$ & -- & -35 & $\begin{array}{ll}5 & 18\end{array}$ & 53 & 3 & 7 & - & 5 & 10 & - & \\
\hline & $10 \mathrm{H}-\mathrm{CC}, 0-3$ & (A) & 94.5 & - & - & - & - & $-1-$ & - & 7 & - & - & - & - & - & - & -- & -1. & - & - & -- & -- & -6 & $6-$ & - & - & - & - & - & - & - & \\
\hline & & (B) & & - & - & - & - & -1 & 18 & & - & - & - & & - & - & - & 5 & - & - & 8 & & -38 & $\begin{array}{ll}8 & 15\end{array}$ & 530 & $\begin{array}{ll}0 & 1 .\end{array}$ & 5 & - & & 4 & & \\
\hline & $11 \mathrm{H}-2,50-53$ & (A) & 96.5 & - & - & - & - & $-1-$ & & & 1. & - & - & - & - & - & - & -1 & - & - - & -- & --1 & - & $7-$ & -- & 8 & 1 & - & - & - & - & \\
\hline & & (B & & - & - & - & - & & & & - & - & - & & - & - & - & 3 & - & -1 & $12-$ & --1 & -25 & $\begin{array}{lll}5 & 11\end{array}$ & 25 & $\begin{array}{ll}5 & 17\end{array}$ & 3 & 5 & 4 & 8 & - & \\
\hline & $11 \mathrm{H}-4,50-53$ & (A) & 99.5 & - & - & - & - & $-1-$ & - & & 5 & - & - & - & - & - & - & -1. & - & - & - & -- & -12 & $2-$ & -- & - & - & - & - & 10 & - & \\
\hline & & (B) & & - & - & - & - & & - & & - & - & - & - & - & - & - & 3 & - & - & $5-$ & --1 & -28 & $\begin{array}{ll}8 & 18\end{array}$ & 40 & 2 & 4 & - & - & 16 & - & \\
\hline & H- $6,50-53$ & (A) & 102.5 & - & - & - & - & $-1-$ & - & 66 & 2 & - & - & - & - & - & - & $-1-$ & - & - - & - & --1 & -3 & $3-$ & -- & - & - & - & - & 4 & - & \\
\hline & & (E) & & - & - & - & - & & - & & - & - & - & - & - & - & -- & $-1-$ & - & -1 & $10-$ & --1 & -22 & 220 & 26 & 5 & 11 & 2 & 2 & 6 & - & \\
\hline & $\mathrm{H}-\mathrm{CC}, 0-3$ & (A) & 104.0 & - & - & - & - & $-1-$ & - & 91 & 1. & - & - & - & - & - & -- & $-1-$ & - & - - & - & -- & -9 & $9-$ & $-\overline{0}$ & - & - & - & - & 3 & - & \\
\hline & & (E & & - & - & - & - & & - & & - & - & - & - & - & - & - & $-1-$ & - & -1 & $12-$ & --1 & -18 & $\begin{array}{ll}8 \quad 12 \\
Y\end{array}$ & 20 & - & 5 & 4 & 5 & 2 & & \\
\hline & $H-2,50-53$ & (A & 106.5 & - & - & - & - & $-1-$ & - & & - & - & - & - & - & - & -- & $-1-$ & - & - & - & -- & -- & -- & -- & $=-$ & -1 & $=$ & - & - & - & \\
\hline & & (B & & - & - & - & - & & & & - & - & - & - & - & - & -- & $-1-$ & - & - & $28-$ & -- & -25 & 10 & 25 & - & 2 & 12 & 9 & 6 & & \\
\hline & $H-4,50$ & (A) & 109.0 & - & - & - - & - & $-1-$ & & & - & - & - & - & - & -- & -- & $-1-$ & - & - & - & -- & -1 & $\bar{n}$ & -- & - & - & - & - & - & - & \\
\hline & & (1. & & - & - & - & - & & & & - & - & & - & - & -- & -- & $-1-$ & - & & 23 & & -20 & $\begin{array}{lll}0 & 23\end{array}$ & $\begin{array}{ll}3 & 19\end{array}$ & 9 & 4 & 15 & - & 3 & & \\
\hline & H-6, 53-56 & (A & 112.0 & - & - & - & - & $-1-$ & - & 31 & - & - & - & - & - & - & -- & $-1-$ & - & - & -- & -- & $5-$ & -2 & $\overline{-1}$ & - & - & - & - & - & 1 & \\
\hline & & & & - & - & & & & & & - & & & - & & & & $-1-$ & - & & $3-$ & & 322 & $\begin{array}{ll}2 & 11\end{array}$ & 17 & - & 3 & 5 . & - & 8 & 3 & \\
\hline & $\mathrm{H}-\mathrm{CC}, 0-3$ & $(t$ & 113.5 & - & - & - & - & $-1-$ & & & - & - & - & - & - & -- & -- & $-1-$ & - & & - & & -2 & - & - & - & - & - & - & 4 & - & \\
\hline & & & & & . & & & & & & - & & & - & & & & $-1-$ & - & & & & - & 5 & 21 & - & 2 & 3. & & 7 & - & \\
\hline & H-3, 55-58 & & & 1- & - & - & - & & & 11 & - & - & -1 & - & - & - - & -- & $-1-$ & - & & & & $1-$ & - & & & & & - & - & & \\
\hline
\end{tabular}


Table 2 (continued).

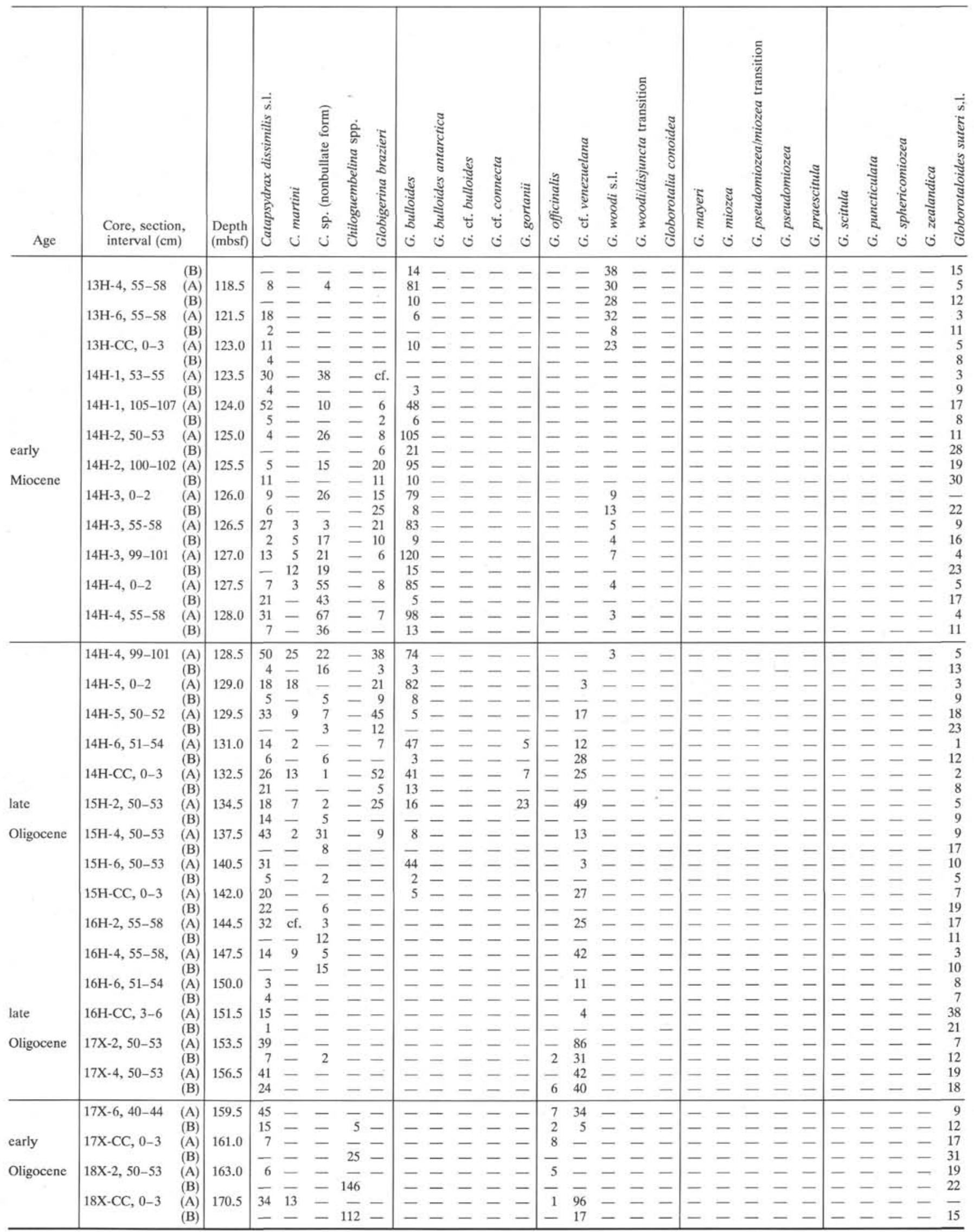

Note: $(\mathrm{A})=>150-\mu \mathrm{m}$-size fraction and $(\mathrm{B})=\langle 150-\mu \mathrm{m}$-size fraction. 
Table 2 (continued).

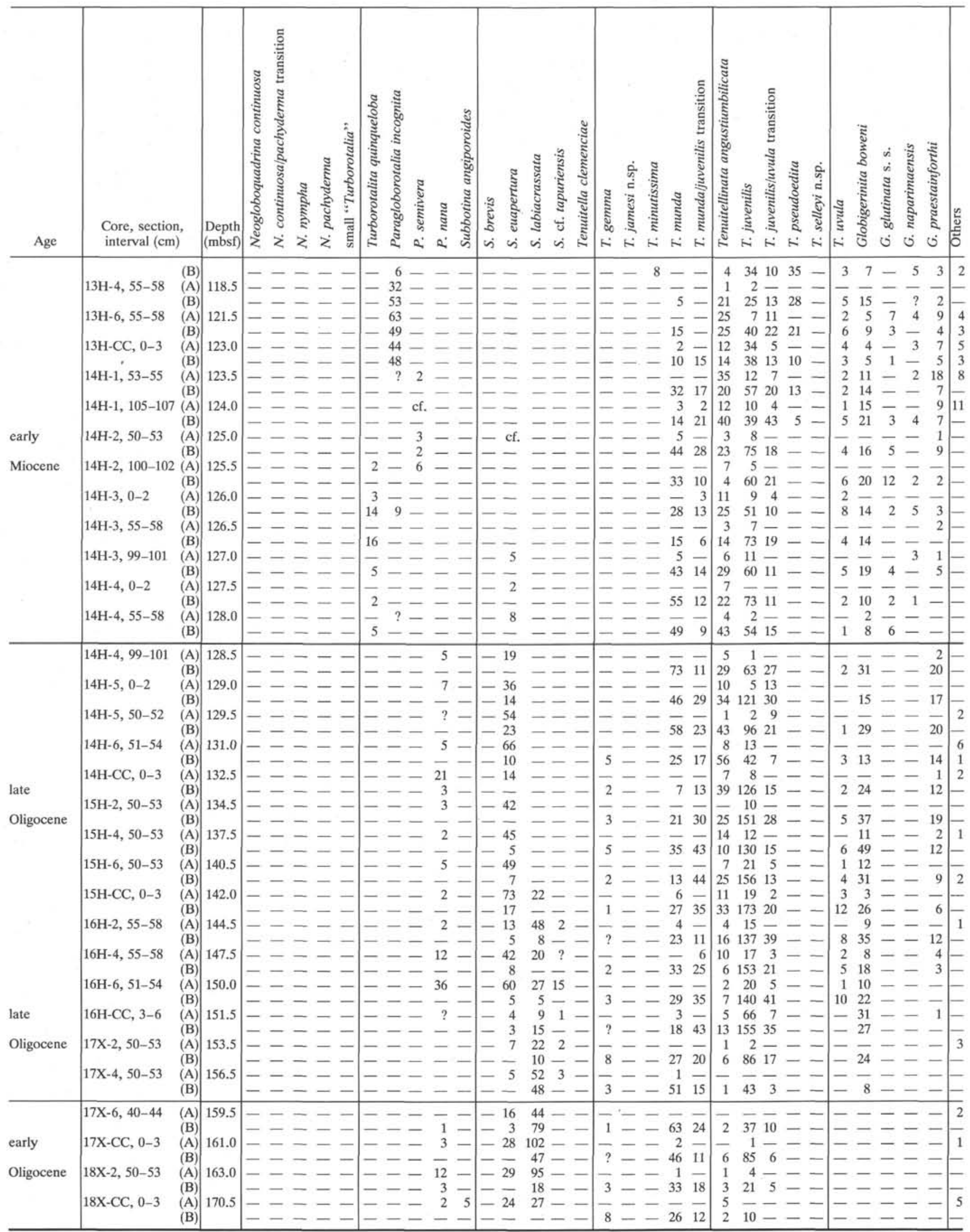


Table 3. Quantitative data of planktonic foraminifers, Hole 749B.

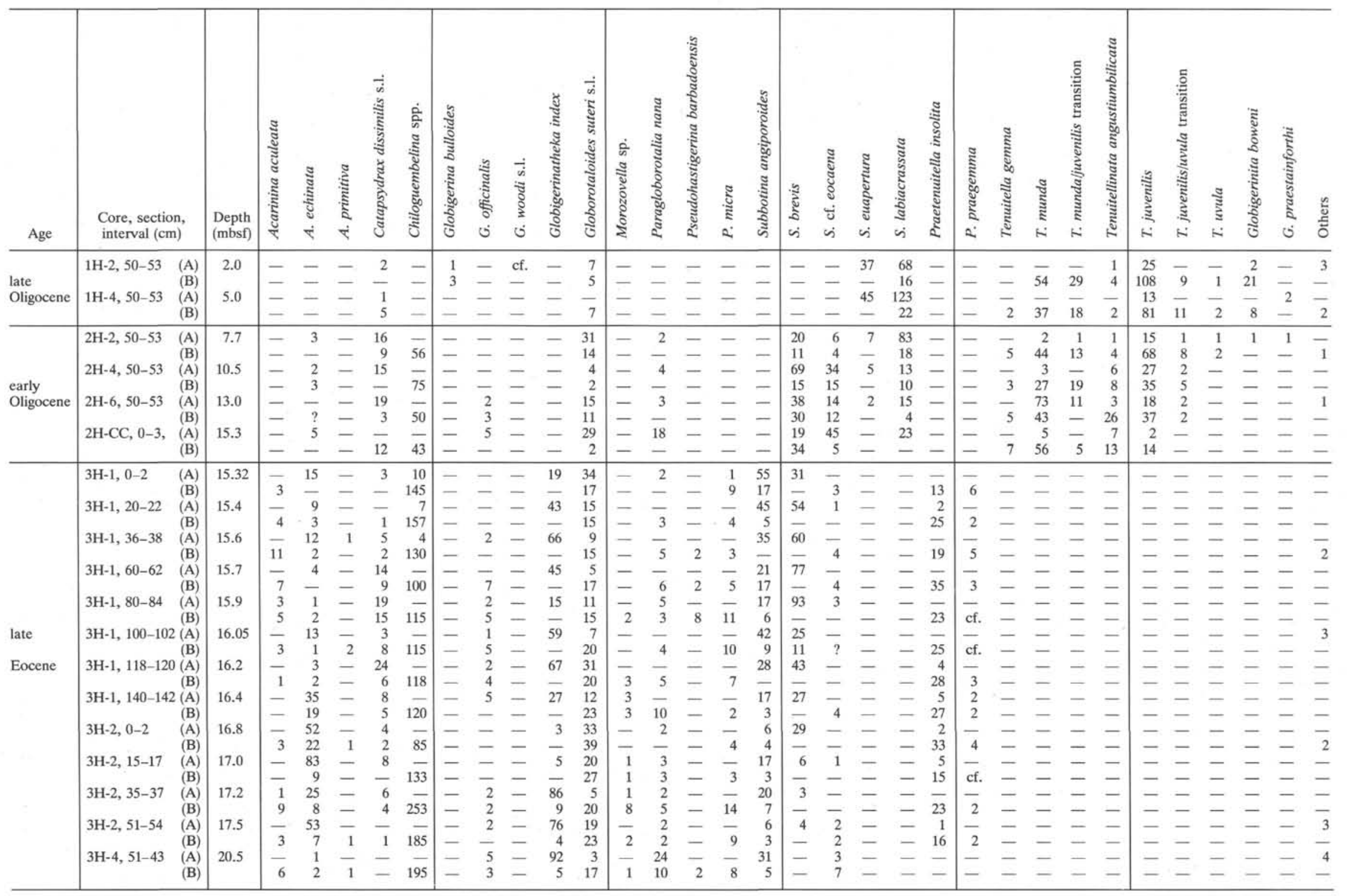

Note: $(\mathrm{A})=<150-\mu \mathrm{m}$-size fraction. 
Table 4. Planktonic foraminifer zonations, Hole 747A

\begin{tabular}{|c|c|c|c|c|}
\hline \multicolumn{2}{|c|}{ Epoch } & \multicolumn{2}{|c|}{ Zone and subzone } & Datum \\
\hline \multicolumn{2}{|l|}{ Pleistocene } & \multirow{3}{*}{ N. pachyderma } & G. antarctica-T. uvula & \multirow{5}{*}{$\begin{array}{l}<\text { FAD G. antarctica } \\
<\text { FAD G. puncticulata } \\
<\text { FAD G. sphericomiozea } \\
<\text { LAD G. sphericomiozea }\end{array}$} \\
\hline \multirow{3}{*}{ Pliocene } & late & & G. puncticulata & \\
\hline & \multirow{2}{*}{ early } & & G. bulloides & \\
\hline & & \multicolumn{2}{|c|}{ G. sphericomiozea } & \\
\hline \multirow{6}{*}{ Miocene } & late & \multicolumn{2}{|c|}{ G. scitula } & \\
\hline & \multirow{2}{*}{ middle } & \multicolumn{2}{|c|}{ N. nympha } & \multirow{5}{*}{$\begin{array}{l}<\text { FAD } G \text {. scitula } \\
<\text { FAD } N \text {. continuosa } \\
<\text { LAD } G \text {. zealandica } \\
<\text { FAD } G \text {. praescitula } \\
<\text { LAD } S \text {. euapertura }\end{array}$} \\
\hline & & G. wooc & G. praescitula & \\
\hline & \multirow{2}{*}{ early } & \multicolumn{2}{|c|}{ G. zealandica/G. pseudomiozea } & \\
\hline & & \multicolumn{2}{|c|}{ P. incognital $P$. semivera } & \\
\hline & \multirow{2}{*}{ late } & \multirow{2}{*}{ S. euapertura } & S. labiacrassata & \\
\hline \multirow[t]{2}{*}{ Oligocene } & & & C. cubensis & \multirow{2}{*}{$\begin{array}{l}<\text { LAD Chiloguembelina } \\
<\text { LAD } S \text {. angiporoides }\end{array}$} \\
\hline & early & \multicolumn{2}{|c|}{ S. angiporoides } & \\
\hline
\end{tabular}

Note: As modified from Schlich, Wise, et al. (1989). See Figure 1 for zonal correlation.

\section{TAXONOMIC NOTES}

Praetenuitella insolita (Jenkins) (Plate 1, Figs. 1-3). Jenkins, 1966, p. 1120 , fig. 13 , nos. 113-118. This species is characterized by a smooth wall and a highly arched aperture; it differs from $P$. impariapertura $\mathrm{Li}$ by its almost circular test, a convex dorsal side, and a narrow umbilicus. It has been recorded in the latest Eocene of New Zealand (Jenkins, 1966), equatorial Pacific (Jenkins and Orr, 1972), and Tasman Sea (Jenkins and Srinivasan, 1985). It is a common element in the uppermost Eocene from Hole 749B.

Praetenuitella praegemma Li (Plate 1, Fig. 4). Li, 1987, p. 309, pl. 1, figs. 11-22. Unlike $P$. insolita or $P$. impariapertura, $P$. praegemma possesses a low, extraumbilical aperture. It occurred together with $P$. insolita in the uppermost Eocene from Hole 749B.

Tenuitella gemma (Jenkins) (Plate 1, Fig. 5). Jenkins, 1966, p. 1115, fig. 11, nos. 97-103. Tenuitella gemma is a small $(80-100 \mu \mathrm{m})$ Oligocene species with numerous records throughout the world (Li and Radford, 1991). On the Kerguelen Plateau, it was last recorded in Sample 120-747A-14H-6, 51-54 cm, at a level close to the Oligocene/Miocene boundary. Jenkins and Srinivasan (1985) reported that $T$. gemma occurred together with Praetenuitella insolita in the upper Eocene of Site 592, but no illustration has been given to support this. Keller (1985) recorded Globorotaloides gemma from the upper Eocene in the American Gulf Coast; however, her specimen nos. 5-7 in figure 3 bear a macroperforate and (sparsely) muricate wall, and thus appear to belong to Acarinina rather than to Tenuitella gemma.

Tenuitella munda (Jenkins) (Plate 1, Figs. 6 and 7). Jenkins, 1966, p. 1121 , fig. 14, nos. 126-133. Compared with its ancestor T. gemma, T. munda is large (120-180 $\mu \mathrm{m})$ and has only four chambers in the final whorl. An evolutionary transition from T. munda to Tenuitellinata juvenilis has been observed by Jenkins (1966), Jenkins and Srinivasan (1985), and $\mathrm{Li}$ (1987), and is further confirmed in this study by the presence of intermediate forms linking these two species throughout the range of $T$. munda. Moreover, $T$. munda showed a close affinity with contemporaneous $T$. angustiumbilicata in its $4 \frac{1}{2}$-chambered variants.

Tenuitella clemenciae (Bermúdez) (Plate 3, Figs. 8 and 9). Bermúdez, 1961 , p. 1321 , pl. 17 , fig. 10 . Small forms referred to this species were found with a diameter of about $100 \mu \mathrm{m}$, compared with 300 $\mu \mathrm{m}$ in the holotype. As both T. clemenciae and T. munda have four chambers in the final whorl, the separation of these two forms is difficult, but they have different stratigraphic ranges and species associations. Tenuitella clemenciae so far has been recorded only in the early and middle Miocene (Zones N7-N10) (Krasheninnikov and Hoskins, 1973, as Globorotalia continuosa; Fleisher, 1974), whereas $T$. munda ranges from the middle Oligocene to earliest Miocene (Kennett and Srinivasan, 1983; this study). This suggests that a gap of about $4 \mathrm{~m}$.y. existed between the LAD of $T$. munda and FAD of $T$. clemenciae, according to the geochronologic scale of Berggren et al. (1985b). Tenuitella clemenciae, therefore, is homeomorphic with, but independent of, $T$. munda. The specimen figured by $\mathrm{Li}(1987$, pl. 2, fig. 9) as atypical $T$. clemenciae from the Oligocene of Trinidad is more likely a variant of $T$. munda rather than $T$. clemenciae.

Tenuitella minutissima (Bolli) (Plate 3, Figs. 4-6). Bolli, 1957, p. 119, pl. 29, fig. 1. The diagnostic characters of $T$. minutissima are its minute test $(90-120 \mu \mathrm{m})$, rapidly enlarging chambers, and a flat dorsal side. It is a minor component in the early to middle Miocene.

Tenuitella jamesi n.sp. (Plate 4, Figs. 8-11). See below for systematic description. This new species differs from $T$. minutissima in having an elongate final chamber which, like that in Turborotalita quinqueloba (Plate 4, Fig. 7), extends onto the umbilicus. It was a minor constituent in late early Miocene to early middle Miocene faunas.

Tenuitellinata angustiumbilicata (Bolli) (Plate 1, Figs. 9-10). Bolli, 1957 , p. 109, pl. 22, figs. 12 and 13. This taxon can be differentiated by its robust, five-chambered test $(120-180 \mu \mathrm{m})$ and axiointraumbilical aperture $(\mathrm{Li}, 1987$, p. 311). It was common in the middle Oligocene to earliest Miocene and occurred together with Tenuitella munda and Globigerinita praestainforthi.

Tenuitellinata juvenilis (Bolli) (Plate 2, Figs. 3-6). Bolli, 1957, p. 100, pl. 24 , figs. 5 and 6 . At high magnification, this pustulate species is distinguished from species of Globigerina, which possess a typical spinose wall (see Plate 2, Figs. 1-2). The view of Jenkins (1966, 1985 ), that this species is an ancestor of Globigerinita glutinata, rather than a junior synonym, is upheld here, because forms referable to $T$. juvenilis occurred as early as the middle Oligocene, whereas bullate $G$. glutinata s.s. did not appear until the early Miocene (Li, 1987; Spezzaferri and Premoli Silva, 1991; this study). After evolving from $T$. munda in the middle Oligocene, $T$. juvenilis rapidly became a common, sometimes large $(>250 \mu \mathrm{m})$, microperforate species in younger sequences.

Tenuitellinata pseudoedita (Subbotina) (Plate 3, Figs. 2 and 3). Subbotina et al., 1960, p. 55, pl. 11. figs. 1-3. The species concept of $\mathrm{Li}(1987$, p. 312) applied to $T$. cf. T. pseudoedita is followed here, but the name $T$. pseudoedita is used. Tenuitellinata pseudoedita is distinguished from $T$. angustiumbilicata by the smaller test (about $100 \mu \mathrm{m}$ ) and anteriointraumbilical, rather than axiointraumbilical aperture. It is the dominant microperforate species in the early to middle Miocene.

Tenuitellinata selleyi n.sp. (Plate 4, Figs. 1-4). This new species differs from $T$. pseudoedita in its smooth rather than pustulate wall. It is a minor component in the lower middle Miocene from Hole 747A. See systematic description below.

Tenuitellinata uvula (Ehrenberg) (Plate 3, Figs. 10 and 11). Ehrenberg, 1862 , p. 308 ; 1873 , pl. 2, figs. 24 and 25 (fide Rögl, 1985, p. 323). High-spired $T$. uvula is closely linked with low-spired $T$. 


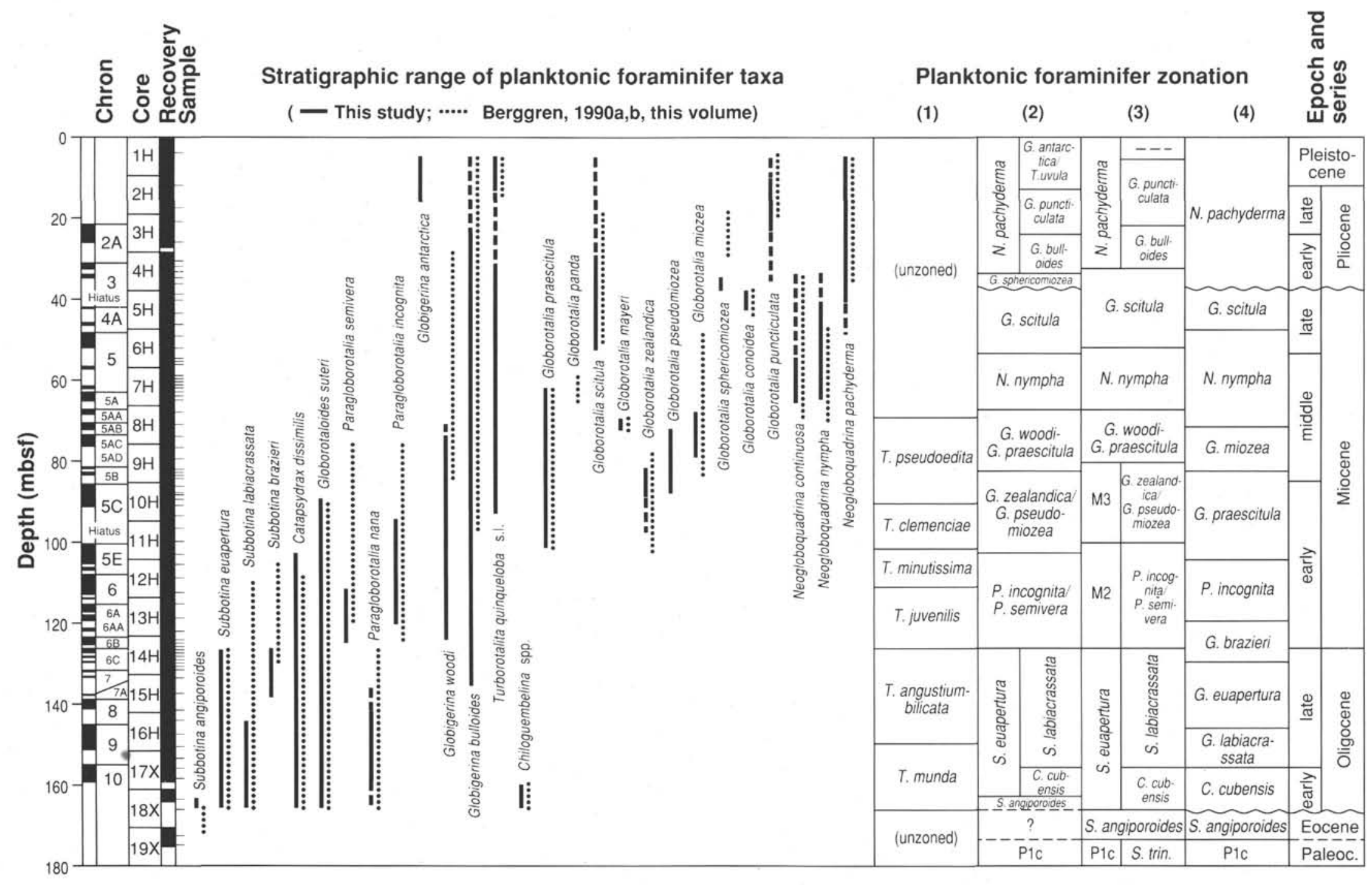

Planktonic foraminifer zonation

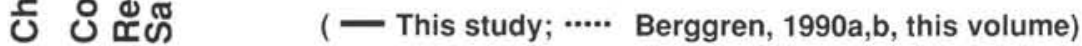

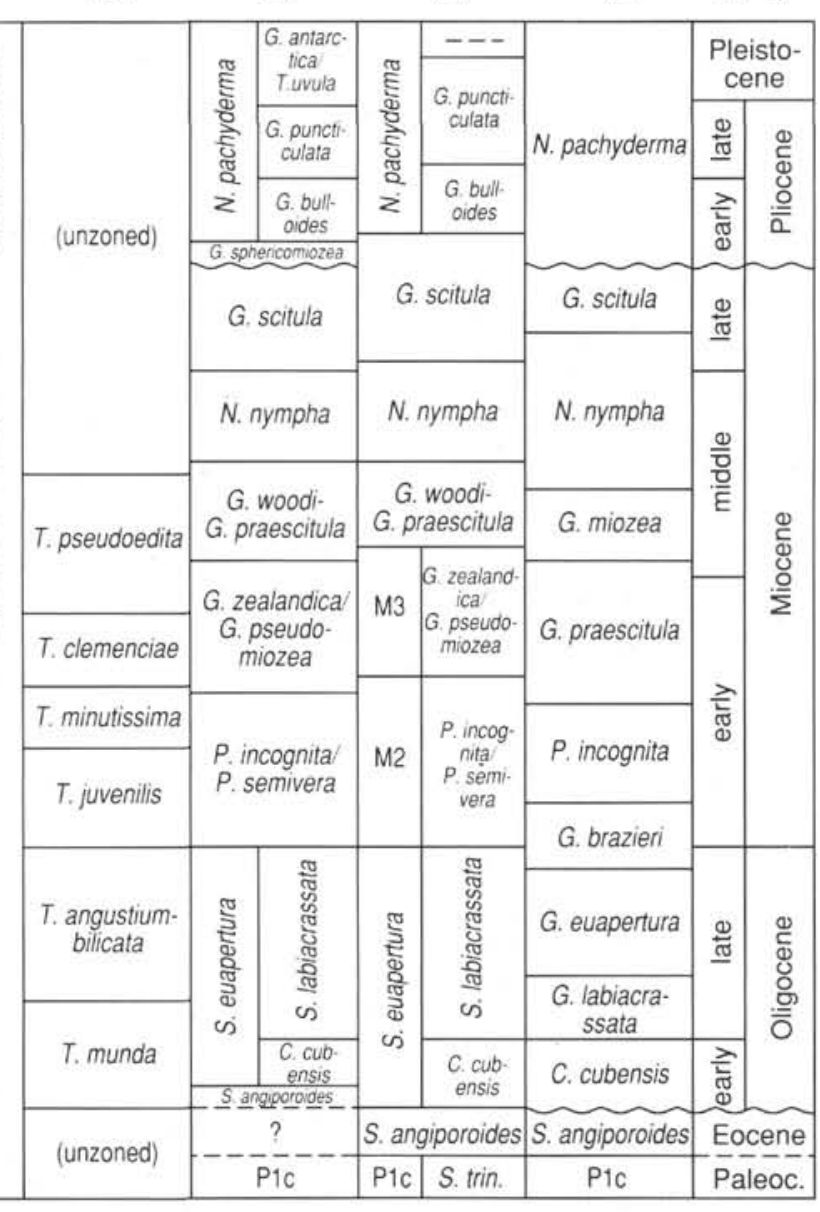

Figure 1. Cenozoic planktonic foraminifer biostratigraphy of Hole 747A, with ranges of selected species. $(1)=$ lineage zonation of the microperforate tenuitellids (see also Fig. 4); (2) = zonation used in this study (see also Table 4); (3) = zonation from Schlich, Wise, et al. (1989); and (4) = zonation from Berggren (this volume a and b). 
Table 5. Planktonic foraminifer zonations, Hole 749B.

\begin{tabular}{|c|c|c|c|c|}
\hline \multicolumn{2}{|c|}{ Epoch } & \multicolumn{2}{|c|}{ Zone and subzone } & Datum \\
\hline \multirow{3}{*}{ Oligocene } & late & \multirow{2}{*}{ S. euapertura } & S. labiacrassata & \multirow{4}{*}{$\begin{array}{l}<\text { LAD Chiloguembelina } \\
<\text { LAD } S \text {. angiporoides } \\
<\text { LAD } P \text {. insolita }\end{array}$} \\
\hline & \multirow{2}{*}{ early } & & C. cubensis (AP14a) & \\
\hline & & \multicolumn{2}{|c|}{ S. angiporoides (AP13) } & \\
\hline \multirow{6}{*}{ Eocene } & \multirow{2}{*}{ late } & \multicolumn{2}{|l|}{$P$, insolita } & \\
\hline & & \multicolumn{2}{|c|}{ G. suteri (AP12) } & \multirow{5}{*}{$\begin{array}{l}<\text { FAD } P \text {. insolita } \\
<\text { LAD } S \text {. linaperta } \\
<\text { LAD } A \text {. primitiva } \\
<\text { FAD } G \text {. index } \\
<\text { FAD } P \text {. micra }\end{array}$} \\
\hline & \multirow{4}{*}{ middle } & \multicolumn{2}{|l|}{ G. index (AP11) } & \\
\hline & & \multicolumn{2}{|c|}{ A. collactea (AP10) } & \\
\hline & & \multicolumn{2}{|l|}{ P. micra (AP9) } & \\
\hline & & \multicolumn{2}{|c|}{ A. bullbrooki (AP8) } & \\
\hline
\end{tabular}

Note: As modified from Schlich, Wise, et al. (1989). See Figure 2 for zonal correlation.

juvenilis by intermediates with a test width:height ratio of about 1:1 (Plate 3, Fig. 7). It occurred sparsely in the late Oligocene and the middle Miocene, but it increased in abundance in the late Miocene and the Pliocene. Tenuitellinata uvula is the only tenuitellid species found in the Pleistocene of Hole 747A.

Globigerinita boweni Brönnimann and Resig (Plate 2, Fig. 7). Brönnimann and Resig, 1971, p. 1271, pl. 26, figs. 1-4. This form may be assigned to $G$. glutinata s.l., but it bears an inflated, bulla-like final chamber (rather than a true bulla) that covers the umbilicus. It first appeared in the late Oligocene, at a level about 5 m.y. earlier than the first appearance of $G$. glutinata s.s.

Globigerinita glutinata (Egger) (Plate 2, Fig. 9). Egger, 1893 (1895), p. 371, pl. 13, figs. 19-21 (fide Saito et al., 1981). Unlike G. boweni, this species possesses a true umbilical bulla that is thin, flat, and sometimes tubelike with more than one opening. It is rare in all samples from its first occurrence in the earliest Miocene. Brummer (1988) and Hemleben et al. (1989) studied the ontogeny of modern microperforate species and concluded that all species but $G$. minuta that have a morphology as $T$. juvenilis in the early stage of ontogeny, including $T$. juvenilis itself, are synonymous with $G$. glutinata. This view is not followed here because nonbullate $T$. juvenilis occurred almost $10 \mathrm{~m} . y$. earlier than bullate G. glutinata, suggesting their independent taxonomic status, although they are phylogenetically closely related. A similar case is seen in Globigerinatella insueta, which also possesses an early ontogeny of a $T$. juvenilis type (see Brönnimann and Resig, 1971, pl. 21 fig. 3).

Globigerinita naparimaensis Brönnimann (Plate 2, Fig. 8). Brönnimann, 1951, p. 16, text figs. 1 and 2 (holotype). This species can be easily recognized by the amphoralike umbilical chamber, with two distinct semicircular apertures, compared with the single slit in $G$. boweni. In our material, G. naparimaensis always occurred together with $T$. juvenilis and $G$. boweni following its first appearance in the earliest Miocene.

Globigerinita praestainforthi Blow (Plate 3, Fig. 1). Blow, 1969, p. 383, pl. 25 , figs. 3-5. $\mathrm{Li}(1987$, p. 311$)$ suggested that this species was closely related to Tenuitellinata pseudoedita. The holotype $(280 \mu \mathrm{m})$ and the specimens found here $(150-200 \mu \mathrm{m})$, however, are all larger than the small-tested $T$. pseudoedita $(<120 \mu \mathrm{m})$. Globigerinita praestainforthi is now also considered to be related to the equally largetested, contemporaneous $T$. angustiumbilicata, and these both became extinct simultaneously in the early Miocene.

Globigerinatella sp. (Plate 4, Figs. 5 and 6). Specimens with a spherical test bearing circular apertural openings are considered to be juveniles of Globigerinatella insueta, which occurred only sparsely in the late early Miocene.

\section{DESCRIPTION OF NEW SPECIES}

\section{Genus TENUITELLA Fleisher, 1974}

Tenuitella jamesi n. sp. Li, Radford, and Banner (Plate 4, Figs. 8-11)

Description. Test small, low trochospiral, $5-5 \frac{1}{2}$ chambers in the final whorl, increasing rapidly in height, final chamber elongate and extending onto the umbilicus; wall microperforate and pustulate; aperture a slitlike opening, extraumbilical.

Remarks. Although the final chamber is similar to that of the spinose Turborotalita quinqueloba (Natland), Tenuitella jamesi is distinguished by its microperforate and pustulate wall. A form described as Globorotalia sp. 4 by Jenkins and Orr (1972, pl. 18, figs. $7-12)$ and as Tenuitella sp. by Fleisher (1974, p. 1134) and Li (1987, p. 311 ), differs from $T$. jamesi in the huge lip, but both have a similar range in the Miocene. This species is named in honor of Dr. Keith James, geologist of the Shell International Petroleum Company, The Hague.

Distribution. Tenuitella jamesi ranged from the lower middle Miocene to the middle middle Miocene in Hole 747A.

Size. Maximum diameter 100-130 $\mu \mathrm{m}$; holotype, $122 \mu \mathrm{m}$.

Holotype. 030873 (Plate 4, Figs. 8 and 9).

Paratypes. 031119 (Plate 4, Fig. 10), 031013 (Plate 4, Fig. 11).

Type locality. Southern Indian Ocean, Section 120-747A-11H-CC $(0-3 \mathrm{~cm})$ (holotype), Samples $120-747 \mathrm{~A}-10 \mathrm{H}-4,52-55 \mathrm{~cm}$, and $-8 \mathrm{H}-4$, $55-58 \mathrm{~cm}$ (paratypes).

\section{Genus TENUITELLINATA Li, 1987}

Tenuitellinata selleyi $\mathrm{n}$. sp. Li, Radford, and Banner (Plate 4, Figs. 1-4)

Description. Test small, low trochospiral, five chambers in the final whorl, enlarging slowly; wall microperforate and smooth, but may be pustulate in umbilical area; aperture a low arch, with a thin lip, anteriointraumbilical.

Remarks. Tenuitellinata selleyi differs from other tenuitelline species in having a small test, a smooth wall, and a low-arched, anteriointraumbilical aperture. In size, it is closely related to $T$, pseudoedita. The partially smooth wall may represent an iterative phenomenon, being typical of the Eocene Praetenuitella, because all other Oligocene-Miocene tenuitellids have been described as pustulate $(\mathrm{Li}, 1987)$.

This species is named after Professor Richard Selley, of the Geology Department, Imperial College, London.

Distribution. Tenuitellinata selleyi occurred in the lower middle Miocene of Hole 747A.

Size. Maximum diameter 100-120 $\mu \mathrm{m}$; holotype, $108 \mu \mathrm{m}$.

Holotype. 030871 (Plate 4, Figs. 1 and 2).

Paratypes. 030869 (Plate 4, Fig. 3), 031034 (Plate 4, Fig. 4).

Type locality. Southern Indian Ocean, Section 120-747 A-11H-CC $(0-3 \mathrm{~cm})$ (holotype), Samples 120-747A-12H-2, 50-53 cm, and -8H-6, $55-58 \mathrm{~cm}$ (paratypes).

\section{LINEAGE ZONATION OF TENUITELLID FORAMINIFERS}

The Oligocene and Miocene planktonic foraminifer faunas in the study area are dominated by microperforate, pustulate tenuitellids, with potential for further biostratigraphic refinement. To achieve a detailed correlation, a lineage zonation of 


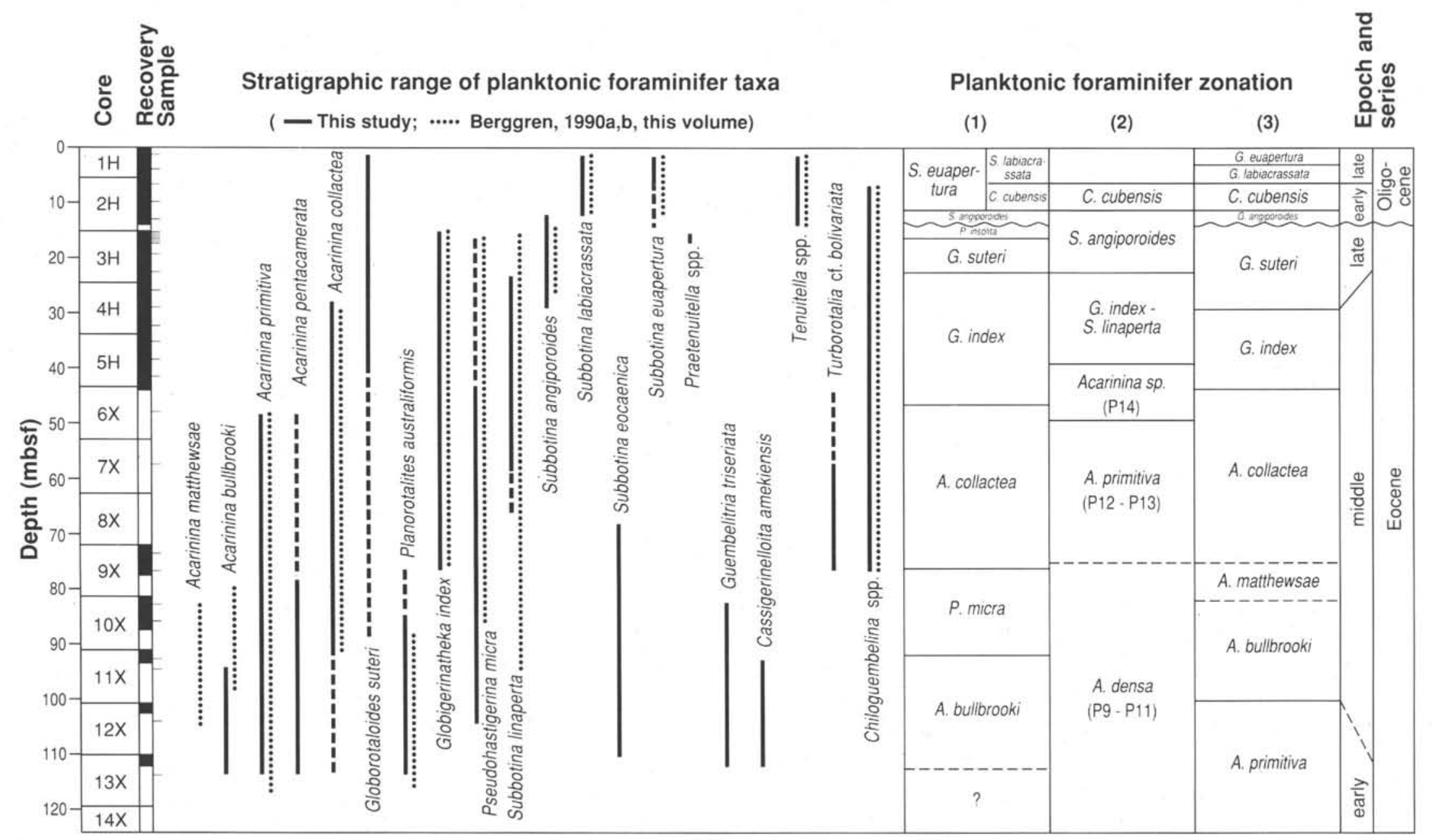

Figure 2. Planktonic foraminifer biostratigraphy of Hole 749B, with ranges of selected species. (1) = revised zonation of Stott and Kennett $(1990)$ used in this study; (2) $=$ zonation from Schlich, Wise, et al. (1989); and (3) = zonation from Berggren (this volume $a$ and b). See Table 3 for details of tenuitellid species in the latest Eocene and Oligocene (Cores $120-749 \mathrm{~B}-1 \mathrm{H}$ through $-3 \mathrm{H})$. 
Table 6. Characteristics of microperforate planktonic foraminifer.

\begin{tabular}{|c|c|c|c|c|c|c|}
\hline Genera & Type species & Test/coiling & $\begin{array}{l}\text { Chambers in } \\
\text { last whorl and } \\
\text { surface texture }\end{array}$ & Aperture & $\begin{array}{l}\text { Immediate } \\
\text { ancestor }\end{array}$ & Range \\
\hline${ }^{\mathrm{a}}$ Praetenuitella & P. praegemma $\mathrm{Li}$ & Low spired & 4-7; smooth & $\begin{array}{l}\text { High to low, marginal- } \\
\text { extraumbilical }\end{array}$ & Pseudohastigerinia & P16-P17 \\
\hline${ }^{\mathrm{a}}$ Tenuitella & T. gemma (Jenkins) & Low spired & $\begin{array}{l}\text { 4-7; pustulate, } \\
\text { rarely smooth }\end{array}$ & Low, extraumbilical & Praetenuitella & $\mathrm{P} 18-\mathrm{N} 23$ \\
\hline${ }^{\mathrm{a}}$ Tenuitellinata & T. angustiumbilicata (Bolli) & Low to high spired & $4-5$; pustulate & Low, axiointra-umbilical & Tenuitella & $\mathrm{P} 19-\mathrm{N} 23$ \\
\hline${ }^{\mathrm{a}}$ Globigerinita & $\begin{array}{l}\text { G. naparimaensis } \\
\text { Brönnimann }\end{array}$ & Low spired & 4 ; pustulate & With apertural bulla & Tenuitellinata & $\mathrm{P} 21-\mathrm{N} 23$ \\
\hline Tinophodella & $\begin{array}{l}\text { T. ambitacrena Loeblich } \\
\text { and Tappan }\end{array}$ & Low spired & 4; pustulate & $\begin{array}{l}\text { With apertural-sutural } \\
\text { bulla }\end{array}$ & Tenuitellinata & $\mathrm{N} 16-\mathrm{N} 23$ \\
\hline Tenuitellita & T. iota (Parker) & Low spired & 5 ; pustulate & With apertural bulla & Tenuitella & $\mathrm{N} 23$ \\
\hline${ }^{\text {a } G l o b i g e r i n a t e l l a}$ & $\begin{array}{l}\text { G. insueta Cushman and } \\
\text { Stainforth }\end{array}$ & Spherical & $1-2$; pustulate & $\begin{array}{l}\text { Areal and sutural, with } \\
\text { bullae }\end{array}$ & Tenuitellinata & N6-N8 \\
\hline Polyperibola & P. christiani Liska & Spherical & $1-2$; pustulate & Sutural, with bullae & Tenuitellinata & N16 \\
\hline Candeina & C. nitida d'Orbigny & High spired & $3-4 ;$ smooth & Sutural & Tenuitellinata & $\mathrm{N} 16-\mathrm{N} 23$ \\
\hline
\end{tabular}

Note: Biozones after Blow (1969).

${ }^{a}$ Genera found in this study.

the microperforate group is introduced as an alternative scheme for high-latitude planktonic foraminiferal biostratigraphy. Within the lineage framework, two types of biozones have been employed: Total Range Zone and Partial Range Zone (see discussions in Berggren and Miller, 1988). A summary of this lineage zonation and correlation of zones is given in Table 7 and Figures 1 and 4. See Appendix B for an additional early Oligocene Zone, the Tenuitella gemma Partial Range Zone.

\section{Praetenuitella insolita Total Range Zone (late Eocene)}

Definition. Total range of zonal marker and other Praetenuitella species.

Remarks. The zone was first proposed as the Globorotalia insolita Zone by Jenkins and Orr (1972). It lies immediately below the Eocene/Oligocene boundary and is equivalent to Antarctic Zone AP12 (upper part) (Stott and Kennett, 1990), or tropical Zones P16-P17 (Blow, 1969, 1979; Berggren and Miller, 1988).

\section{Tenuitella munda Partial Range Zone (late Oligocene)}

Definition. Partial range of the nominate taxon from the last occurrence (LO) of Chiloguembelina spp. (base) to the first occurrence (FO) of Tenuitellinata uvula (top).

Remarks. In addition to the zonal marker, other microperforate species also common in the zone include Tenuitellinata juvenilis and Tenuitellinata angustiumbilicata. It is equivalent to Antarctic Zone AP14b or tropical Zone P21b.

\section{Tenuitellinata angustiumbilicata Partial Range Zone (late Oligocene)}

Definition. Partial range of the nominate taxon from the FO of Tenuitellinata uvula (base) to the FO of both Globigerinita glutinata s.s. and Globigerinita naparimaensis (top).

Remarks. In addition to the nominate taxon, Tenuitellinata juvenilis is also common, and Globigerinita praestainforthi has its FO in the zone. It is equivalent to tropical Zone P22.

\section{Tenuitellinata juvenilis Partial Range Zone (early Miocene)}

Definition. Partial range of the nominate taxon from the FO of Globigerinita glutinata s.s. (base) to the LO of Tenuitellinata angustiumbilicata (top).

Remarks. Within the zone, Tenuitella munda becomes extinct and both Tenuitellinata pseudoedita and Tenuitella minutissima make their first appearances. It is equivalent to tropical Zones N4-N5.

\section{Tenuitella minutissima Partial Range Zone (early Miocene)}

Definition. Partial range of the nominate taxon from the LO of Tenuitellinata angustiumbilicata (base) to the FO of Tenuitella clemenciae.

Remarks. In addition to the nominate taxon, other common species include Tenuitellinata juvenilis and Tenuitellinata pseudoedita. In tropical areas, the base of this zone can be defined also by the FO of Globigerinatella insueta. It is equivalent to tropical Zone N6.

\section{Tenuitella clemenciae Partial Range Zone (early Miocene)}

Definition. Partial range of the nominate taxon from its FO (base) to that of $T$. jamesi (top).

Remarks. Other species characterizing the zone include Tenuitellinata juvenilis, Tenuitellinata pseudoedita, and Globigerinita spp. Tenuitellinata selleyi first appeared near the base of the zone. It is equivalent to tropical Globigerinatella insueta Zone (N7).

\section{Tenuitellinata pseudoedita Partial Range Zone (middle Miocene)}

Definition. Partial range of the nominate taxon from the FO of Tenuitella jamesi (base) to the LO of the nominate taxon (top).

Remarks. Tenuitellinata juvenilis and Globigerinita spp. are also common in the zone. Not only the nominate taxon but also other low-spired tenuitellines including Tenuitella minutissima, Tenuitella clemenciae, and Tenuitella jamesi become extinct at the top of the zone. In Hole 747A this extinction level lies in Anomaly Correlative (Chron) $5 \mathrm{~A}$, or within Zone N12, based on the geochronological correlation of Berggren et al. (1985a, 1985b). Evidence from previous studies, however, shows that the extinction level of Tenuitella minutissima, Tenuitella clemenciae, and related forms in tropical areas, are within Zone N10 (Fleisher, 1974; Li, 1987), a level about 2 m.y. earlier than that recorded here.

\section{PALEOCEANOGRAPHIC IMPLICATION OF TENUITELLID PLANKTONIC FORAMINIFERS}

Being abundant in high latitudes, the microperforate tenuitellid foraminifers have been recently used as cold-water indexes in paleoceanographic studies (Boersma et al., 1987; Premoli Silva and Boersma, 1988; Spezzaferri and Premoli Silva, 1991). During the Oligocene macroperforate and spinose species (e.g., of Globigerina) dominated tropical areas, whereas the microperforate species dominated cooler environments, although both groups occur together in marine sequences of that age and show a worldwide distribution pattern (Li and Radford, 1991). 


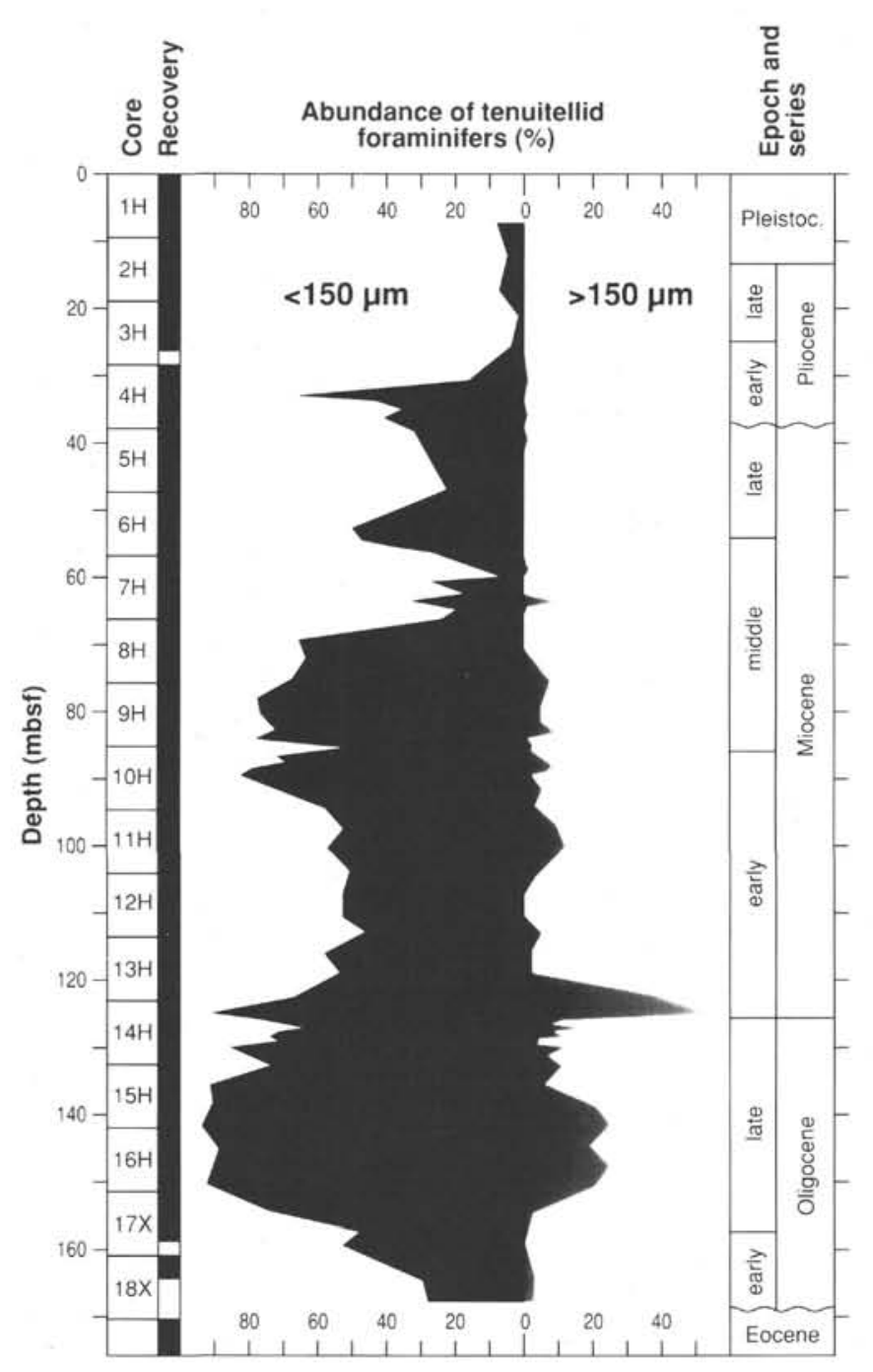

Figure 3. Total composition of microperforate tenuitellid planktonic foraminifers, Hole $747 \mathrm{~A}$. The absolute abundance, with $50 \%-90 \%$ of the fauna in the $<150-\mu \mathrm{m}$-size fractions, occurred from the late Oligocene to the middle Miocene. Also note that specimens $>150 \mu \mathrm{m}$ are mainly restricted to the late Oligocene and the earliest Miocene.

A decrease in surface-water temperatures from $11^{\circ} \mathrm{C}$ in the late Eocene to about $7^{\circ} \mathrm{C}$ in the earliest Oligocene was recorded in subantarctic regions (Shackleton and Kennett, 1975). It has been proposed that the Antarctic ice cap first developed during this time (Kennett and Barker, 1990; Zachos et al., this volume). This event probably stimulated the evolution of Praetenuitella species from Pseudohastigerina and the transition from Praetenuitella to Tenuitella (Li, 1987). This process was completed in the earliest Oligocene with the appearance of Tenuitella gemma, the smallest and the first typical microperforate tenuitelline species (Jenkins, 1966). Subsequent evolutionary appearances of Tenuitella munda and Tenuitellinata species could have been the response to a stabilizing environment and increasing food supply (e.g., Shackleton, 1987), leading to the establishment and differentiation of the microperforate tenuitellid group within the planktonic foraminifer realm.

In the late Oligocene and the early Miocene, the fourchambered Tenuitellinata juvenilis was dominant at Hole $747 \mathrm{~A}$ and it is characteristic of high latitudes, whereas the five-chambered Tenuitellinata angustiumbilicata represents the lineage in low latitudes (Kennett et al., 1985; Li, 1987; Spezzaferri and Premoli Silva, 1991). This supports the view that the evolution of test architecture was influenced not only by biological but also by microenvironmental factors, as has been recognized from modern species studies (e.g., Hemleben et al., 1989), although the extent of this influence is not clear. Documentation of these morphological variations from different localities thus appears to be essential for a better understanding of the relationship between species evolution and paleoceanography.

We observed two major events in the early Miocene: (1) extinctions of the typical Oligocene species, including Tenuitella munda, Tenuitellinata angustiumbilicata, and Globigerinita praestainforthi; and (2) the first appearances of such typical Miocene species as Tenuitella minutissima and Globigerinatella insueta (low latitudes only). Apparently the former event appears to be related to a brief cooling ( $22 \mathrm{Ma})$, and the latter to the brief warming (about $20 \mathrm{Ma}$ ) (Table 8).

Extinctions of typical Miocene species, such as Tenuitella minutissima and Tenuitellinata pseudoedita, occurred from 14 to $13 \mathrm{Ma}$ in the middle Miocene, a period of major global cooling and further development of the ice cap on Antarctica (Miller et al., 1987; Kennett and Barker, 1990). These lowspired species with an extraumbilical or anteriointraumbilical aperture probably could not adapt to cooling surface water, with temperatures falling to about $5^{\circ} \mathrm{C}$ (Shackleton and Kennett, 1975). High-spired species with axiointraumbilical apertures, especially Tenuitellinata juvenilis and $T$. uvula, however, appear to have successfully withstood these conditions.

A high abundance $(35 \%-50 \%)$ of surviving Tenuitellinata and Globigerinita species was recorded in the latest Miocene to earliest Pliocene, broadly correlating with the evolution of both Candeina nitida and Polyperibola christiani in tropical areas. Evidence from other planktonic foraminifers as well as other fossil groups indicates that global warming was the main feature of this interval, resulting in the retreat of the Antarctic ice cap, marine transgression, and isolation of the Mediterranean (Berggren and Haq, 1976; Kennett, 1977; Berggren and Olsson, 1986; Wright and Thunell, 1988).

In the latest Pliocene (about $3 \mathrm{Ma}$ ), Tenuitellinata juvenilis completely disappeared from the study area, although $T$. uvula still survived with only a minimum occurrence. The planktonic foraminifer fauna then was dominated by leftcoiling Neogloboquadrina pachyderma, a cold-water indicator (Ericson, 1959). This decline of the tenuitellids coincides with, and may be attributed to, global cooling, causing the northward expansion of ice rafting in the Southern Oceans and the development of a Northern Hemisphere ice sheet. During this time, a minimum isotopic temperature and a maximum ice volume for the entire Cenozoic were recorded (Margolis et al., 1975; Kennett, 1977; Shackleton, 1987). This trend changed only slightly in the Pleistocene, as indicated by the slow recovery of $T$. uvula (Fig. 4).

Table 8 demonstrates the correlations between major events in paleoceanography and microperforate planktonic foraminifers. Apparently, species extinctions or appearances coincided with major cooling or warming events. The majority of species, once abundant, are good indicators of cool to cold waters; provincial temperature gradients in post-Oligocene time favored warm-water species evolution.

\section{CONCLUSIONS}

Quantitative analyses of the microperforate tenuitellid planktonic foraminifers from Holes $747 \mathrm{~A}$ and $749 \mathrm{~B}$ reveal that this group was predominant in the Oligocene and Miocene and is of great potential value in biostratigraphy. Based on the first 


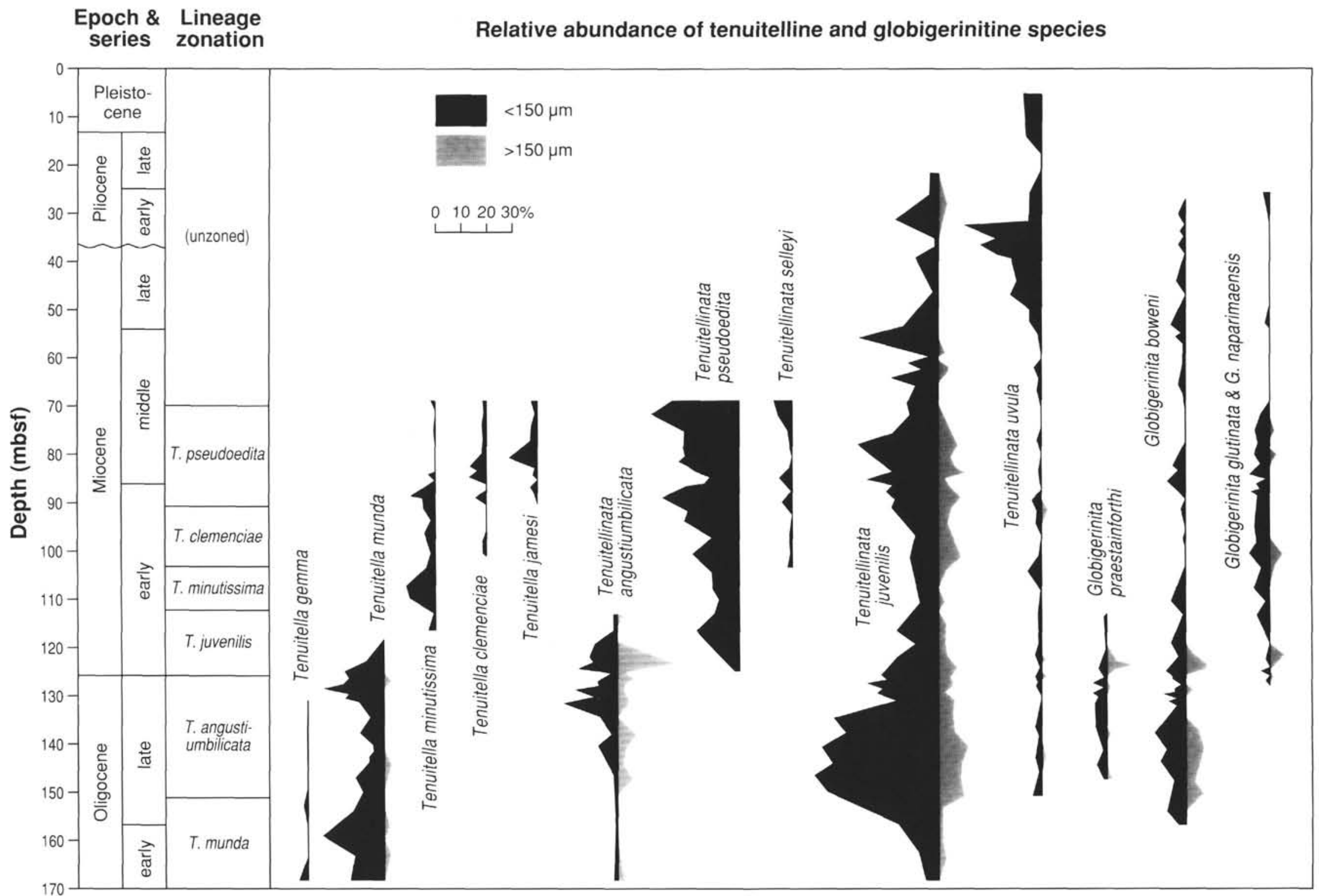

Figure 4. Abundances of tenuitellid species, Hole 747A. Two extinction levels, in the early Miocene and the middle Miocene, are significant for biostrati-graphic and paleoceanographic interpretations. 
Table 7. Correlation between tropical and Antarctic zonations and lineage zonation of the tenuitellid foraminifers.

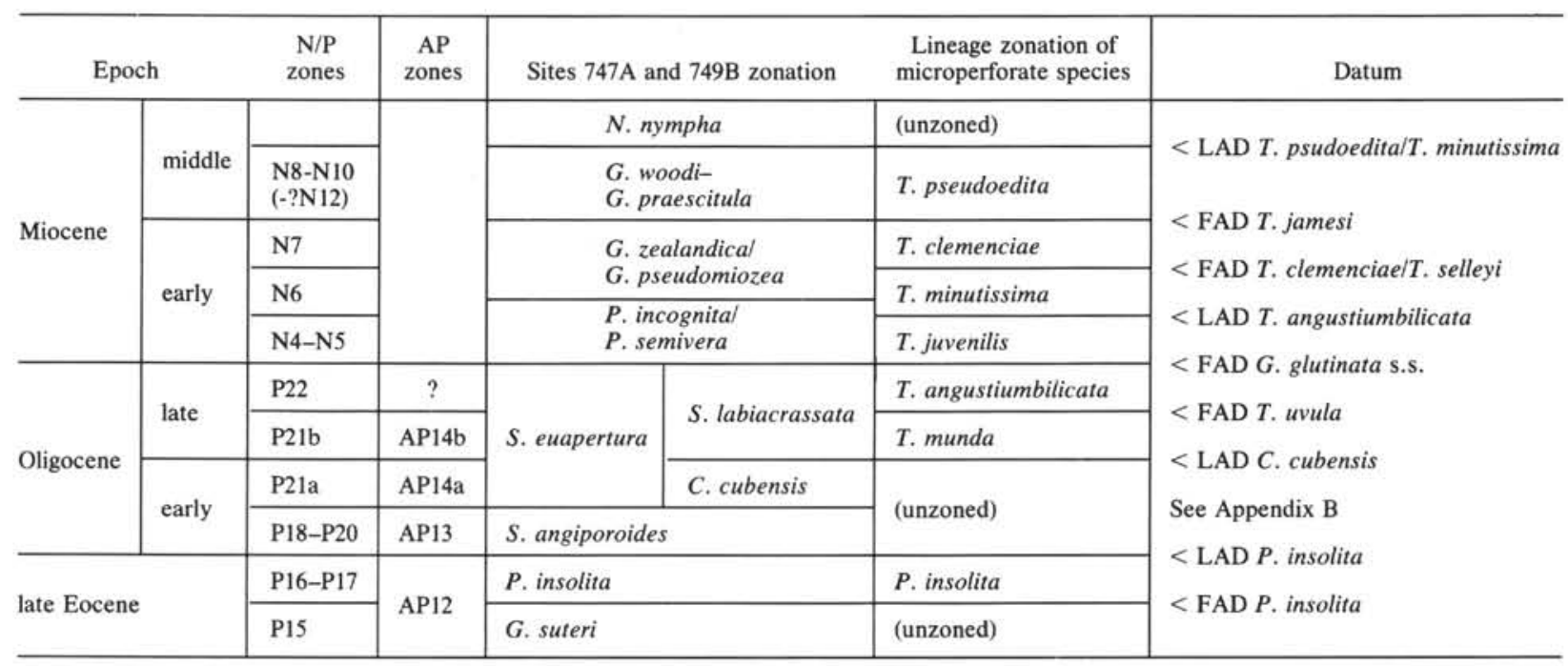

Notes: N/P zones after Blow (1969, 1979), Berggren et al. (1985a, 1985b), and Berggren and Miller (1988). AP zones from Stott and Kennett (1990). Note that the early Oligocene is unzoned because its inadequate recovery was caused by a hiatus across the Eocene/Oligocene boundary, but the microperforate fauna of this age in other localities is dominated by Tenuitella gemma.

Table 8. Event correlations of microperforate planktonic foraminifers to paleoceanography.

\begin{tabular}{|c|c|c|}
\hline Age/interval & Paleoceanographic events & $\begin{array}{l}\text { Microperforate planktonic } \\
\text { foraminferal events }\end{array}$ \\
\hline $\begin{array}{l}3 \mathrm{Ma}, \\
\text { latest Pliocene }\end{array}$ & $\begin{array}{l}\text { Global cooling; maximum ice volume in } \\
\text { Cenozoic; Northern Hemisphere ice } \\
\text { sheet developed. }\end{array}$ & $\begin{array}{l}\text { T. juvenilis disappeared and minimum } T \text {. } \\
\text { uvula in Site 747A. }\end{array}$ \\
\hline $\begin{array}{l}\text { 6-4.5 Ma, } \\
\text { latest Miocene to } \\
\text { early Pliocene }\end{array}$ & $\begin{array}{l}\text { Warming; Antarctic ice cap retreat; } \\
\text { marine transgression; isolation of } \\
\text { Mediterranean. }\end{array}$ & $\begin{array}{l}\text { High frequency of surviving species; } \\
\text { Candeina and Polyperibola occurred in } \\
\text { tropics. }\end{array}$ \\
\hline $\begin{array}{l}\text { 10-9 Ma, } \\
\text { late Miocene }\end{array}$ & $\begin{array}{l}\text { Further cooling; sea level fall; large } \\
\text { volume of Antarctic ice. }\end{array}$ & $\begin{array}{l}\text { Low frequency of surviving } T \text {. juvenilis } \\
\text { and } T \text {. uvula. }\end{array}$ \\
\hline $\begin{array}{l}\text { 14-13 Ma, } \\
\text { middle Miocene }\end{array}$ & $\begin{array}{l}\text { Global cooling; East Antarctic ice cap } \\
\text { increased in size. }\end{array}$ & Extinctions of typical Miocene species. \\
\hline $\begin{array}{l}\text { 20-19 Ma, } \\
\text { early Miocene }\end{array}$ & $\begin{array}{l}\text { Warming; circumglobal equatorial } \\
\text { circulation ends; Indo-Pacific passage } \\
\text { closing. }\end{array}$ & Appearance of typical Miocene species. \\
\hline $\begin{array}{l}22 \mathrm{Ma} \text {, } \\
\text { early Miocene }\end{array}$ & $\begin{array}{l}\text { Cooling; drop in surface water } \\
\text { temperature. }\end{array}$ & Extinctions of typical Oligocene species. \\
\hline $\begin{array}{l}\text { 34- } 33 \mathrm{Ma} \text {, } \\
\text { early Oligocene }\end{array}$ & $\begin{array}{l}\text { Warming; vigorous bottom-water } \\
\text { circulation; extensive deep-sea erosion. }\end{array}$ & $\begin{array}{l}\text { Transition between Tenuitella and } \\
\text { Tenuitellinata. }\end{array}$ \\
\hline $\begin{array}{l}\text { 37- } 36 \mathrm{Ma} \text {, } \\
\text { earliest Oligocene }\end{array}$ & $\begin{array}{l}\text { Maximum cooling; East Antarctic ice cap } \\
\text { developed. }\end{array}$ & First typical Tenuitella (T. gemma). \\
\hline $\begin{array}{l}\text { 38- } 37 \mathrm{Ma} \text {, } \\
\text { latest Eocene }\end{array}$ & Further cooling; cool surface water. & Praetenuitella evolved. \\
\hline $\begin{array}{l}\text { 50- } 39 \mathrm{Ma} \text {, middle-late } \\
\text { Eocene }\end{array}$ & $\begin{array}{l}\text { Warm surface-water temperature } \\
\text { decreasing. }\end{array}$ & $\begin{array}{l}\text { a(Pseudohastigerina micra retreating } \\
\text { from Antaractic water) }\end{array}$ \\
\hline
\end{tabular}

zand/or last appearance datums of species, a lineage zonation is proposed as an alternative scheme for refinement of the planktonic foraminifer biostratigraphy in high latitudes. The lineage biozones can be correlated with other planktonic foraminifer zonations commonly used today, such as the standard tropical N/P zones and other high-latitude zonations.

Large accumulations of microperforate species indicate the influence of cold-water masses. Morphological changes leading to appearances or extinctions of species appear to be related directly to major paleoceanographic events, especially warming or cooling. Between the subantarctic and the tropical regions, the species composition was basically the same in the latest Eocene and Oligocene, but distinctly different in the Miocene to Pleistocene. The Leg 120 material lacks warmwater inhabitants, such as Globigerinatella insueta (lower Miocene), Polyperibola christiani (upper Miocene), and Candeina nitida (upper Miocene to Holocene). This suggests that the provincial distribution of tenuitellid species began only since Miocene time.

\section{ACKNOWLEDGMENTS}

W. A. Berggren (shipboard micropaleontologist) kindly invited us to conduct this study. We thank the ODP curator and other staff for collecting and shipping samples. Critical 
reviews of this manuscript by R. L. Fleisher and G. Keller are gratefully acknowledged. A. R. Brown drew the text figures. Q. Li and S. Radford were supported by the Hui Fellowship at Imperial College.

\section{REFERENCES}

Berggren, W. A., Aubry, M.-P., and Hamilton, N., 1983. Neogene magnetostratigraphy of Deep Sea Drilling Project Site 516 (Rio Grande Rise, South Atlantic). In Barker, P. F., Carlson, R. L., Johnson, D. A., et al., Init. Repts. DSDP, 72: Washington (U.S. Govt. Printing Office), 675-713.

Berggren, W. A., and Haq, B. U., 1976. The Andalusian stage (late Miocene): biostratigraphy, biochronology and paleoecology. Palaeogeogr. Palaeoclimatol. Palaeoecol., 20:67-129.

Berggren, W. A., Kent, D. V., and Flynn, J. J., 1985a. Jurassic to Paleogene: Part 2. Paleogene geochronology and chronostratigraphy. In Snelling, N. J. (Ed.), The Chronology of the Geological Record. Geol. Soc. London Mem., 10:141-195.

Berggren, W. A., Kent, D. V., and Van Couvering, J. A., 1985b. The Neogene: Part 2. Neogene geochronology and chronostratigraphy. In Snelling, N. J. (Ed.), The Chronology of the Geological Record. Geol. Soc. London Mem., 10:211-260.

Berggren, W. A., and Miller, K. G., 1988. Paleogene tropical planktonic foraminiferal biostratigraphy and magnetobiochronology. Micropaleontology, 34:362-380.

Berggren, W. A., and Olsson, R. K., 1986. North Atlantic Mesozoic and Cenozoic paleobiogeography. In Vogt, P. R., and Tucholke, B. E. (Eds.), The Geology of North America (Vol. M): Geol. Soc. Am., 565-587.

Bermúdez, P. J., 1961. Contribuciön al estudio de las Globigerinidae de la regiön Caribe-Antillana (Paleocene-Recente). Mem. III Congr. Geol. Venezolano, Soc. Geol. Venez., Bol. Geol., Publ. Espec., 3:1119-1393.

Blow, W. H., 1969. Late middle Eocene to Recent planktonic foraminiferal biostratigraphy. In Brönniman, P., and Renz, H. H. (Eds.), Proceedings of the First International Conference on Planktonic Microfossils, Geneva: Leiden (E. J. Brill), 1:199-422. 1979. The Cainozoic Globigerinida: Leiden (E. J. Brill).

Boersma, A., Premoli Silva, I., and Shackleton, N. J., 1987. Atlantic Eocene planktonic foraminiferal paleohydrographic indicators and stable isotope paleoceanography. Paleoceanography, 2:287-331.

Bolli, H. M., 1957. Planktonic foraminifera from the OligoceneMiocene Cipero and Lengua formations of Trinidad, B.W.I. In Loeblich, A. R., Jr., Tappan, H., Beckmann, J. P., Bolli, H. M., Gallitelli, E. M. and Troelsen, J. C. (Eds.), Studies in Foraminifera. Bull. U.S. Nat. Mus., 215:97-124.

Boltovskoy, E., 1974. Neogene planktonic foraminifera of the Indian Ocean (DSDP, Leg 26). In Davies, T. A., Luyendyk, B. P., et al., Init. Repts. DSDP, 26: Washington (U.S. Govt. Printing Office), 675-741.

Brönnimann, P., 1951. Globigerinita naparimaensis, n.gen., n.sp., from the Miocene of Trinidad, B.W.I. Contrib. Cushman Found. Foraminiferal Res., 2:16-18.

Brönnimann, P., and Resig, J., 1971. A Neogene globigerinacean biochronologic timescale of the Southwestern Pacific. In Winterer, E. L., Riedel, W. R., et. al., Init. Repts. DSDP, 7, Pt. 2: Washington (U.S. Govt. Printing Office), 1235-1409.

Brummer, G.-J.A., 1988. Comparative ontogeny of modern microperforate planktonic foraminifers. In Brummer, G.-J.A., and Kroon, D., Planktonic Foraminifers as Tracers of Ocean-climate History: Amsterdam (Free Univ. Press), 77-129.

Ericson, D. B., 1959. Coiling direction of Globigerina pachyderma as a climatic index. Science, 130:219-220.

Fleisher, R. L., 1974. Cenozoic planktonic foraminifera and biostratigraphy, Arabian Sea, Deep Sea Drilling Project, Leg 23A. In Whitmarsh, R. B., Weser, O. E., Ross, D. A., et al., Init. Repts. DSDP, 23: Washington (U.S. Govt. Printing Office), 1001-1072. 1975. Oligocene planktonic foraminiferal biostratigraphy, Central North Pacific Ocean, DSDP Leg 32. In Larson, R. L., Moberly, R., et al., Init. Repts. DSDP, 32: Washington (U.S. Govt. Printing Office), 753-763.
Haq, B. U., 1984. Paleoceanography: a synoptic overview of 200 million years of ocean history. In Haq, B. u., and Milliman, J. D. (Eds.), Marine Geology and Oceanography of Arabian Sea and Coastal Pakistan: New York (Van Nostrand), 201-231.

Haq, B. U., Hardenbol, J., and Vail, P. R., 1987. Chronology of fluctuating sea levels since the Triassic. Science, 235:1156-1167.

Hemleben, C., Spindler, M., and Anderson, O. R., 1989. Modern Planktonic Foraminifera: Berlin (Springer-Verlag).

Jenkins, D. G., 1966. Planktonic foraminiferal zones and new taxa from the Danian to Lower Miocene of New Zealand. N. Z. J. Geol. Geophys., 8:1088-1126.

1985. Southern mid-latitude Paleocene to Holocene planktic foraminifera. In Bolli, H. M., Saunders, J. B., and PerchNielsen, K. (Eds.), Plankton Stratigraphy: Cambridge (Cambridge Univ. Press), 87-154.

Jenkins, D. G., and Orr, W. N., 1972. Planktonic foraminiferal biostratigraphy of the east equatorial Pacific-DSDP Leg 9. In Hays, J. D., et al., Init. Repts. DSDP, 9: Washington (U.S. Govt. Printing Office), 1059-1193.

Jenkins, D. G., and Srinivasan, M. S., 1986. Cenozoic planktonic foraminifers from the equator to the subantarctic of the southwest Pacific. In Kennett, J. P., von der Borch, C. C., et al., Init. Repts. DSDP, 90: Washington (U.S. Govt. Printing Office), 795-834.

Keigwin, L. D., and Corliss, B. H., 1986. Stable isotopes in late middle Eocene to Oligocene foraminifera. Geol. Soc. Am. Bull., 97:335-345.

Keller, G., 1985. Eocene and Oligocene stratigraphy and erosional unconformities in the Gulf of Mexico and gulf coast. J. Paleontol., 59:882-903.

Kennett, J. P., 1977. Cenozoic evolution of Antarctic glaciation, the circum-Antarctic Ocean, and their impact on global paleoceanography. J. Geophys. Res., 82:3843-3860.

Kennett, J. P., and Barker, P. F., 1990. Latest Cretaceous to Cenozoic climate and oceanographic developments in the Weddell Sea, Antarctica: an ocean-drilling perspective. In Barker, P. F., Kennett, J. P., et al., Proc. ODP, Sci. Results, 113: College Station, TX (Ocean Drilling Program), 937-960.

Kennett, J. P., Keller, G., and Srinivasan, M. S., 1985. Miocene planktonic foraminiferal biogeography and paleoceanographic development of the Indo-Pacific region. In Kennett, J. P. (Ed.), The Miocene Ocean: Paleoceanography and Biogeography: Mem. Geol. Soc. Am., 163:197-236.

Kennett, J. P., and Srinivasan, M. S., 1983. Neogene Planktonic Foraminifera: A Phylogenetic Atlas: Stroudsburg, PA (Hutchinson Ross).

Kennett, J. P., and Vella, P., 1975. Late Cenozoic planktonic foraminifera and paleoceanography at DSDP Site 284 in the cool subtropical South Pacific. In Kennett, J. P., Houtz, R. E., et al., Init. Repts. DSDP , 29: Washington (U.S. Govt. Printing Office), 769-799.

Krasheninnikov, V. A., 1980. Planktonic foraminiferal biostratigraphy of Pliocene and Quaternary deposits of the East Pacific Rise and the Galapagos Spreading Center, Deep Sea Drilling Project Leg 54. In Rosendahl, B. R., Hekinian, R., et al., Init. Repts. DSDP, 54: Washington (U.S. Govt. Printing Office), 509-533.

Krasheninnikov, V. A., and Basov, I. A., 1983. Cenozoic planktonic foraminifers of the Falkland Plateau and Argentine Basin, Deep Sea Drilling Project Leg 71. In Ludwig, W. J., Krasheninnikov, V. A., et al., Init. Repts. DSDP, 71, Pt. 2: Washington (U.S. Govt. Printing Office), 821-858.

Krasheninnikov, V. A., and Hoskins, R. H., 1973. Late Cretaceous, Paleogene and Neogene planktonic foraminifera. In Heezen, B. C., MacGregor, I. D., et al., Init. Repts. DSDP, 20: Washington (U.S. Govt. Printing Office), 105-203.

Krasheninnikov, V. A., and Pflaumann, U., 1978a. Zonal stratigraphy and planktonic foraminifers of Paleogene deposits of the Atlantic Ocean to the west off Africa. In Lancelot, Y., Seibold, E., et al., Init. Repts. DSDP, 41: Washington (U.S. Govt. Printing Office), 581-611.

1978b. Zonal stratigraphy and Neogene deposits of the eastern part of the Atlantic Ocean by means of planktonic foraminifers, Leg 41, Deep Sea Drilling Project. In Lancelot, Y., Seibold, E., et al., Init. Repts. DSDP, 41: Washington (U.S. Govt. Printing Office), 613-657. 
Li, Q., 1987. Origin, phylogenetic development and systematic taxonomy of the Tenuitella plexus (Globigerinitidae, Globigerinina). J. Foraminiferal Res., 17:295-320.

Li, Q., and Radford, S. S., 1991. Evolution and biogeography of Paleogene microperforate planktonic foraminifera. Palaeogeogr. Palaeoclimatol., Palaeoecol., 83:87-115.

Margolis, S. V., Kroopnick, P. M., Goodney, D. E., Dudley, W. C., and Mahoney, M. E., 1975. Oxygen and carbon isotopes from calcareous nannofossils as paleoceanographic indicators. Science, 189:555-557.

Matoba, Y., and Oda, M., 1981. Late Pliocene to Holocene planktonic foraminifers of the Guaymas Basin, Gulf of California, Sites 477 through 481. In Curray, J. R., Moore, D. G., et al., Init. Repts. DSDP, 64: Washington (U.S. Govt. Printing Office), 1003-1026.

Miles, G. A., 1977. Planktonic foraminifera from Leg 37 of the Deep Sea Drilling Project. In Aumento, F., Melson, W. G., et al., Init. Repts. DSDP, 37: Washington (U.S. Govt. Printing Office), 929_ 961.

Miller, K. G., Fairbanks, R. G., and Mountain, G. S., 1987. Tertiary oxygen isotope synthesis, sea-level history, and continental margin erosion. Paleoceanography, 2:1-19.

Poag, C. W., 1972. Neogene planktonic foraminiferal biostratigraphy of the western North Atlantic: DSDP Leg 11. In Hollister, C. D. Ewing, J. I., et al., Init. Repts. DSDP, 11: Washington (U.S. Govt. Printing Office), 483-520.

Poore, R. Z., 1979. Oligocene through Quaternary planktonic foraminiferal biostratigraphy of the North Atlantic: DSDP Leg 49. In Luyendyk, B. P., Cann, J. R., et al., Init. Repts. DSDP, 49: Washington (U.S. Govt. Printing Office), 447-517.

1981. Miocene through Quaternary planktonic foraminifers from offshore southern California and Baja California. In Yeats, R. S., Haq, B. U., et al., Init. Repts. DSDP, 63: Washington (U.S. Govt. Printing Office), 415-436.

, 1984. Middle Eocene through Quaternary planktonic foraminifers from the southern Angola Basin: Deep Sea Drilling Project Leg 73. In Hsü, K. J., LaBrecque, J. L., et al., Init. Repts. DSDP, 73: Washington (U.S. Govt. Printing Office), 429-448.

Premoli Silva, I., and Boersma, A., 1988. Atlantic Eocene planktonic foraminiferal historical biogeography and paleohydrographic indices. Palaeogeogr., Palaeoclimatol., Palaeoecol., 67:315-356.

Quilty, P. G., 1976. Planktonic foraminifera, DSDP Leg 34. In Yeats, R. S., Hart, S. R., et al., Init. Repts. DSDP, 34: Washington (U.S. Govt. Printing Office), 629-703.

Rögl, F., 1985. Late Oligocene and Miocene planktic foraminifera of the Central Paratethys. In Bolli, H. M., Saunders, J. B., and Perch-Nielsen, K. (Eds.), Plankton Stratigraphy: Cambridge (Cambridge Univ. Press), 315-328.

Saito, T., Thompson, P. R., and Breger, D., 1981. Systematic Index of Recent and Pleistocene Planktonic Foraminifera: Tokyo (Univ. of Tokyo).

Salvatorini, G., and Cita, M. B., 1979. Miocene foraminiferal biostratigraphy, DSDP Site 397 (Cape Bojador, North Atlantic). In von Rad, U., Ryan, W.B.F., et al., Init. Repts. DSDP, 47, Pt. 1: Washington (U.S. Govt. Printing Office), 371-373.

Schlich, R., Wise, S. W., Jr., et al., 1989. Proc. ODP, Init Repts., 120: College Station, TX (Ocean Drilling Program).

Shackleton, N. J., 1986. Paleogene stable isotope events. Palaeogeogr., Palaeoclimatol., Palaeoecol., 57:91-102.

1987. The carbon isotope record of the Cenozoic: history of organic carbon burial and of oxygen in the ocean and atmosphere. In Brooks, J., and Fleet, A. J. (Eds.), Marine Petroleum Source Rocks. Geol. Soc. Spec. Publ. London, 26:423-434.

Shackleton, N. J., and Kennett, J. P., 1975. Paleotemperature history of the Cenozoic and the initiation of Antarctic glaciation: oxygen and carbon isotope analyses in DSDP Sites 277, 279 and 281. In Kennett, J. P., Houtz, R. E., et al., Init. Repts. DSDP, 29: Washington (U.S. Govt. Printing Office), 743-755.

Snyder, S. W., and Waters, V. J., 1984. Cenozoic planktonic foraminiferal biostratigraphy of the Goban Spur region, Deep Sea Drilling Project Leg 80. In de Graciansky, P. C., Poag, C. W., et al., Init. Repts. DSDP, 80, Pt. 1: Washington (U.S. Govt. Printing Office), 439-472.
Spezzaferri, S., and Premoli Silva, I., 1991. Oligocene planktonic foraminiferal biostratigraphy and paleoclimatic interpretation from Hole 538A, DSDP Leg 77, Gulf of Mexico. Palaeogeogr., Palaeoclimatol., Palaeoecol., 83:217-263.

Stone, S. M., and Keller, G., 1985. Planktonic foraminiferal biostratigraphy of eastern equatorial Pacific sediments, Deep Sea Drilling Project Leg 85. In Mayer, L., Theyer, F., Thomas, E., et al., Init. Repts. DSDP, 84: Washington (U.S. Govt. Printing Office), 419471

Stott, L. D., and Kennett, J. P., 1990. Antarctic Paleogene planktonic foraminifer biostratigraphy: ODP Leg 113, Sites 689 and 690 . In Barker, P. F., Kennett, J. P., et al., Proc. ODP, Sci. Results, 113: Washington (U.S. Govt. Printing Office), 549-569.

Subbotina, N. N., 1953. Fossil foraminifers of the USSR: Globigerinidae, Hantkeninidae, Globorotaliidae. Trudy VNIGRI, new series, 76 (in Russian). Translated into English by E. Lees, Fossil foraminifera of the USSR; Globigerinidae, Hantkeninidae and Globorotaliidae: London and Wellingborough (Collet's Ltd.), 1971.

Subbotina, N. N., Pishvanova, L. S., and Ivanova, L. V., 1960. Stratigrafiya oligotsenovikh i miotsenovikh otlozheniy Predkarpatya po foraminiferam. Mikrofauna SSSR, 11, Trudi VNIGRI, 153:5-127.

Tjalsma, R. C., 1977. Cenozoic foraminifera from the South Atlantic, DSDP Leg 36. In Barker, P., Dalziel, I.W.D., et al., Init. Repts. DSDP, 36: Washington (U.S. Govt. Printing Office), 493-517.

Wright, J. D., and Thunell, R. C., 1988. Neogene planktonic foraminiferal biogeography and paleoceanography of the Indian Ocean. Micropaleontology, 34:193-216.

Date of initial receipt: 31 January 1990

Date of acceptance: 28 July 1990

MS 120B-171

\section{APPENDIX A}

Checklist of Microperforate Tenuitellid Planktonic Foraminifers Brönnimann and Resig, 1971

Globigerina multiloba (pl. 6, fig. 9), early Miocene (N7-N8). Not G. multiloba Romeo. It may be included in Tenuitellinata pseudoedita in this study.

Candeina nitida (pl. 14, figs. 7-8), Pleistocene (N23).

Globigerinatella insueta (pl. 21, figs. 1-5), early Miocene (N6/N8). Typical specimens with Tenuitellinata juvenilis morphology in early ontogeny (fig. 3).

Globigerinita uvula (pl. 22, figs. 6-9), early Miocene (figs. 6 and 9) and late Pliocene (fig. 7). Now Tenuitellinata uvula. Figure 8 is a smooth, finely perforate form and should be excluded.

Globigerinita flparkerae n.nom. (pl. 23, figs. 1-5), Pleistocene (N23). The name Globigerinita parkerae (= Globigerinoides parkerae Bermédez) is retained (see Kennett and Srinivasan, 1983). G. flparkerae is thus a junior synonym.

Turborotalia iota (pl. 23, figs. 6-8), Pleistocene (N22). Now Tenuitellita iota. Figures 6 and 7 have a cancellate wall and should be excluded.

Globigerinita boweni n.sp. (pl. 26, figs. 1-4), late Oligocene (P22).

Globorotalia (Turborotalia) gemma (pl. 32, figs. 2-3), early Oligocene. Now Tenuitella gemma.

Globorotalia (Turborotalia) nkbrowni n.sp. (pl. 40, figs. 1-8), late Oligocene. Now Tenuitella nkbrowni.

$$
\text { Jenkins and Orr, } 1972
$$

Candeina nitida (pl. 1, figs. 1-4), Pliocene (N19/N20). Figure 3 shows pustules on the early chamber surface.

Globigerina angustiumbilicata (pl. 4, figs. 4-6), early Miocene (N4). Figure 6 now Tenuitellinata angustiumbilicata. Figures 4 and 5 are possibly juveniles of Globigerina angulisuturalis.

Globigerina bradyi (pl. 4, figs. 7-9), early Miocene (N7). Now Tenuitellinata uvula.

Globigerina angustiumbilicata (pl. 5, figs. 6-8), early Oligocene (P20). Now Tenuitellinata angustiumbilicata. 
Globigerina foliata (pl. 7, fig. 6), early Miocene (N7). Not G. foliata Bolli, but a medium-high spired Tenuitellinata juvenilis.

Globigerina eamesi (pl. 8, figs. 1-3), early Miocene (N4). Not G. eamesi Blow, but a Tenuitellinata juvenilis.

Globigerina cf. eamesi (pl. 8, figs. 4-6), late Oligocene (P22). Considered as Tenuitellinata pseudoedita in this study.

Globigerina juvenilis (pl. 10, figs. 1-5), Pleistocene (N22-N23). Now Tenuitellinata juvenilis. Figures 4 and 5 belong to Globigerinita parkerae.

Globigerinatella insueta (pl. 12, figs. 1-3), early Miocene.

Globigerinita glutinata (pl. 17, fig. 1), Pleistocene (N22-N23). Considered as Globigerinita naparimaensis in this study.

Globorotalia sp. 4 (pl. 18, figs. 7-12), middle Miocene (N9). Now Tenuitella sp. See also T. jamesi in this study.

Globorotalia gemma (pl. 22, figs. 7-11), early Oligocene (P20/P21). Now Tenuitella gemma.

Globorotalia insolita (pl. 25, figs. 3-8), latest Eocene. Now Praetenuitella insolita.

Globorotalia cf. minutissima (pl. 27, figs. 3-8), late Oligocene (figs. 3, 4 , and 8 ) and early Miocene (figs. 5-7). Figure 3 is now $T$. pseudoedita, figures 4 and 8 are now Tenuitella gemma, and figure 7 is an early $T$. minutissima. Figures 5 and 6 are duplicates of Tenuitellinata angustiumbilicata in plate 5, figures 6 and 8 .

Poag, 1972

Candeina? parkerae? (pl. 7, figs. 3-5), middle Miocene (N14-N15). Considered as juveniles of Candeina nitida in this study.

Krasheninnikov and Hoskins, 1973

Candeina nitida (pl. 4, figs. 5-6), early Pliocene.

Globigerina angustiumbilicata (pl. 5, figs. 8-10), early Miocene (N5-N7). Considered as Tenuitellinata pseudoedita in this study.

Globigerina bradyi (pl. 6, figs. 7-8), early Miocene. Now Tenuitellinata uvula.

Globigerina bulbosa (pl. 6 figs. 9-11), late Miocene. Not G. bulbosa LeRoy (see Blow, 1969, pl. 13, figs. 3-4), but a variant of Tenuitellinata juvenilis.

Globigerina juvenilis (pl. 8, fig. 6), early Miocene. Now Tenuitellinata juvenilis.

Globigerina praebulloides (pl. 9, figs. 7-12), early Miocene (figs. 10-12) and late Miocene (figs. 7-9). Not G. praebulloides Blow, but variants of Tenuitellinata juvenilis.

Globigerinatella insueta (pl. 12, fig. 3), early Miocene.

Globigerinita glutinata (pl. 12, fig. 6), late Miocene.

Globigerinita incrusta (pl. 12, figs. 7-8), early Miocene.

Globigerinita naparimaensis (pl. 12, fig. 9), late Miocene. Considered as $G$. ambitacrena in this study.

Globigerinita aff. stainforthi (pl. 12, figs. 10-11), early Miocene. Not Catapsydrax stainforthi Bolli, Loeblich and Tappan, but variants of $G$. glutinata.

Globorotalia continuosa (pl. 19, figs. 10-12), early Miocene (N8). Not $G$. continuosa Blow, but considered here as Tenuitella clemenciae

Fleisher, 1974

Globigerinita glutinata (pl. 9, figs. 1-2), Pleistocene (N22). Regarded as Tenuitellinata juvenilis in this study.

Globigerinita glutinata ambitacrena (pl. 9, fig. 3), Pleistocene (N22).

Globigerinita boweni (pl. 9, fig. 4), early Miocene (N7).

Tenuitella gemma (pl. 17, figs. 4, 6, and 7), early Oligocene (P18-P19).

Tenuitella sp. (pl. 17, fig. 5), early Miocene (N10).

Tenuitella clemenciae (pl. 17, fig. 8), early Miocene (N10).

Tenuitella anfracta (pl. 17, figs. 9-10), Pleistocene (N22). Now Tenuitella fleisheri.

Boltovskoy, 1974

Candeina nitida (pl. 1, fig. 4), late Pliocene (N21). Note the specimen with small pustules.

Globigerina juvenilis (pl. 1, fig. 13), Pliocene-Pleistocene (N21-N22). Now Tenuitellinata juvenilis.

Globigerinita glutinata (p. 719, pl. 3, fig. 4), late Pliocene (N21).

Kennett and Vella, 1975

Globigerinita glutinata (pl. 8, figs. 11-13), late Pliocene. Figure 12 is a Tenuitellinata juvenilis.
Globigerinita uvula (pl. 8, fig. 14), early Pliocene. Considered as transitional between Tenuitellinata juvenilis and $T$. uvula.

Fleisher, 1975

Globigerinita boweni (pl. 3, fig. 1), late Oligocene (P22).

Tenuitella clemenciae (pl. 3, figs. 2-4), early Oligocene (figs. 3-4) and late Oligocene (fig. 2). Considered as Tenuitella munda in this study.

Tenuitella gemma (pl. 3, figs. 5-7), early Oligocene (P20).

Quilty, 1976

Globigerina angustiumbilicata (pl. 1, figs. 12-15), early Oligocene. Now Tenuitellinata angustiumbilicata. Figures 14 and 15 show four-chambered Globigerina ciperoensis.

Globigerina bulbosa (pl. 1, figs. 20-21), early Miocene (N8/N9). As Tenuitellinata juvenilis in this study, but not Globigerina bulbosa LeRoy.

Globigerina gortanii gortanii (pl. 3, figs. 1-2), early Oligocene (N2/ N3). Not G. gortanii (Borsetti), but transitional between Tenuitellinata juvenilis and T. uvula.

Globigerina ouachitaensis group (pl. 3, fig. 10), early Oligocene (P19). Not $G$. ouachitaensis Howe and Wallace, but a typical specimen of Tenuitella gemma.

Globigerina praebulloides group (pl. 3, figs. 15-16), early Miocene (fig. 15) and middle Miocene (fig. 16). Not G. praebulloides Blow; both are considered as Tenuitellinata juvenilis. Compare this specimen with figures $I$ and 2 in the same plate.

Catapsydrax boweni (pl. 7, figs. 9-10), early Miocene (N8). A typical C. unicavus. Not Globigerinita boweni Brönnimann and Resig.

Globigerinita ambitacrena (pl. 8, figs. 4-5), middle Miocene (fig. 4) and Pleistocene (fig. 5).

Globigerinita glutinata (pl. 8, figs. 6-11), early-middle Miocene. Figures 6-8 and 10-11 represent variations of Tenuitellinata juvenilis, but figure 9 is a typical Tenuitella clemenciae.

Globigerinita incrusta (pl. 8, figs. 14-16), middle Miocene (N9-N10).

Globigerinita uvula (pl. 8, fig. 18), middle Miocene (N9). Now Tenuitellinata uvula.

Globigerinatella insueta (pl. 8, fig. 22), early Miocene (N8).

Globigerinatella insueta (pl. 9, figs. 1-2), early Miocene (N8). Intermediate form between Tenuitellinata juvenilis and Globigerinatella insueta.

Globorotalia (Turborotalia) clemenciae (pl. 11, figs. 13-14), early Oligocene (P19). Now Tenuitella munda.

Globorotalia (Turborotalia) gemma (pl. 12, fig. 7), early Oligocene (N2/N3). Now Tenuitella gemma.

Globorotalia (Turborotalia) insolita (pl. 12, figs. 11-13), late Eocene. Now Praetenuitella insolita. Typical specimens.

Globorotalia (Turborotalia) minutissima (pl. 13, figs. 3-4), middle Miocene (N10/N11). Now Tenuitella minutissima.

Globorotalia (Turborotalia) cf. obesa (pl. 13, figs. 8-9), middle Miocene (N9). Not Globorotalia obesa Bolli, but a primitive specimen of Tenuitella sp. Turborotalita iota (pl. 16, figs. 12-13), ?Pleistocene. Now Tenuitellita iota.

Clavigerinella nazcaensis $\mathrm{n}$. sp. paratype (pl. 18, fig. 1 only), early Oligocene ( $\mathrm{N} 2 / \mathrm{N} 3)$. Transitional form between Tenuitella munda and Tenuitellinata juvenilis.

Tjalsma, 1977

Turborotalia munda (pl. 4, figs. 7-11), Oligocene. Now Tenuitella munda, but figure 9 is an intermediate between $T$. munda and Tenuitellinata juvenilis.

\section{Miles, 1977}

Candeina nitida (pl. 1, fig. 1), late Miocene. Note the pustules on early chambers.

Globigerinita glutinata (pl. 2, figs. 11-13), late Pliocene. Figure 12 is a Tenuitellinata juvenilis.

Globigerinita uvula (pl. 2, fig. 14), late Miocene. Now Tenuitellinata uvula.

Krasheninnikov and Pflaumann, 1978a

Globorotalia gemma (pl. 5, figs. 9-11), Oligocene. Now Tenuitella gemma. Typical specimens. 
Krasheninnikov and Pflaumann, 1978b

Globigerina bradyi (pl. 1, figs. 5-7), late Miocene. Now Tenuitellinata uvula.

Globigerina juvenilis (pl. 1, figs. 8-9), early Miocene. Now Tenuitellinata juvenilis.

Globigerinatella insueta (pl. 1, figs. 10-11), early Miocene.

Salvatorini and Cita, 1979

Globigerinatella insueta (pl. 2, fig. 16), early Miocene (N8).

Turborotalia sp. (pl. 2, figs. 17-20), late Miocene (N16). Here considered as Tenuitellinata juvenilis.

Turborotalia sp. (pl. 8, figs. 16-19), late Miocene (N16). Here regarded as Tenuitella clemenciae, although this has never been reported from the late Miocene.

Turborotalia clemenciae (pl. 8, figs. 23-24), early Miocene (fig. 24) and late Miocene (fig. 23). Here considered as Tenuitella sp.

\section{Poore, 1979}

Globorotalia minutissima (pl. 4, figs. 7-9), early Miocene (N6). Now Tenuitella minutissima with six chambers in the final whorl.

Globigerinita praestainforthi (pl. 15, figs. 7-8), early Oligocene (P21).

Globorotalia munda (pl. 20, figs. 8-10), early Oligocene (P19/P20). Now Tenuitella munda.

Globorotalia cf. G. munda (pl. 20, figs. 11-13), early Oligocene (P21). Here considered as Tenuitella munda.

Krasheninnikov, 1980

Globigerinoides tenellus (pl. 2, fig. 1), Pleistocene. Not Globigerinoides tenellus Parker, but a typical Globigerinita parkerae.

Globigerinoides(?) sp. (pl. 2, fig. 3), Pleistocene. Here regarded as Globigerinita parkerae.

Globigerinita glutinata (pl. 2, figs. 5-10), Pleistocene. Now Globigerinita (Tinophodella) ambitacrena.

Candeina nitida (pl. 2, figs. 11-12), Pleistocene.

Poore, 1981

Globorotalia minutissima (pl. 2, figs. 1-3), middle Miocene (N11). Here considered as Tenuitella sp.

\section{Matoba and Oda, 1981}

Globigerinita glutinata (pl. 2, fig. 12), Quaternary. Now Tenuitellinata juvenilis.

Globigerinita uvula (pl. 2, fig. 13), Quaternary. Now Tenuitellinata uvula. Turborotalita cf. parkerae (pl. 5, fig. 16), Quaternary. Here considered as Tenuitella fleisheri.

Turborotalita iota (pl. 5, figs. 17-20), Quaternary. Now Tenuitellita iota.

\section{Krasheninnikov and Basov, 1983}

Globorotalia gemma (pl. 10, figs. 6-9), Oligocene. Now Tenuitella gemma.

Globorotalia munda (pl. 10, figs. 10-12), ?late Eocene. Now Tenuitella munda, but figure 11 is a typical Globorotaloides suteri.

Globigerinita glutinata (pl. 13, figs. 1-2), Neogene-Quaternary. Here considered as Globigerinita naparimaensis.

Globigerinita uvula (pl. 13, figs. 6-8), Neogene-Quaternary. Now Tenuitellinata uvula.

\section{Poore, 1984}

Globorotalia gemma (pl. 3, figs. 1-4), early Oligocene (OL1). Now Tenuitella gemma.

Globigerina angustiumbilicata (pl. 3, figs. 5-7), early Oligocene (OL2). Now Tenuitellinata angustiumbilicata, but figure 7 is transitional between Tenuitella gemma and $T$. munda.

\section{Snyder and Waters, 1984}

Globorotalia postcretacea (pl. 3, figs. 12-14), early Oligocene (P18/ P19). Here referred to Tenuitella neoclemenciae Li.

\section{Stone and Keller, 1985}

Globigerina angustiumbilicata (pl. 9, figs. 6-7), early Miocene (N4c). Here considered as Tenuitellinata pseudoedita.

Globigerinita glutinata s.1. (pl. 10, fig. 14), early Pliocene (N18). Here as Globigerinita naparimaensis.

Globigerinita uvula (pl. 10, fig. 15), early Miocene (N7-N8). Now Tenuitellinata uvula.

Globigerinita iota (pl. 10, fig. 16), Pleistocene (N23). Now Tenuitellita iota.

Jenkins and Srinivasan, 1985

Globorotalia gemma (pl. 2, figs. 12-14), early Oligocene. Now as Tenuitella gemma.

Globorotalia insolita (pl. 3, figs. 3-4), late Eocene, G. insolita zone. Now Praetenuitella insolita.

Globorotalia munda (pl. 4, figs. 4-5), late Oligocene. Now Tenuitella munda.

G. munda and G. juvenilis transitional forms (pl. 4, figs. 6-8), late Oligocene. Figure 7 is not a microperforate form and should be excluded.

Globigerina juvenilis (pl. 4, fig. 9), late Oligocene. Here as Tenuitellinata juvenilis.

\section{APPENDIX B}

Further Investigations by S. S. Radford

Further investigations on the lineage of tenuitellid foraminifers indicate the necessity for an additional zone in the lower Oligocene of the Kerguelen Plateau, where an unzoned interval is shown in Table 7 and Figure 1 (this study). This interval lies between the upper Eocene Praetenuitella insolita Zone and the Tenuitella munda Zone of the upper Oligocene.

Tenuitella gemma (Jenkins) was recorded in Sites 747 and 749 (Berggren, this volume; this study) and in Site 748 (Berggren, this volume). It is the earliest representative of the genus and, therefore, marks the evolutionary first appearance of Tenuitella. Li (1987) concluded that this taxon was "directly descended from Praetenuitella (late Eocene)" and gave a range for T. gemma of lower Oligocene (P18-P21).

The following zone is here proposed for the lower Oligocene of the Kerguelen Plateau and should be incorporated within the lineage zonation proposed by $\mathrm{Li}$ et al (Table 7 and Fig. 1, this volume).

\section{Tenuitella gemma Partial Range Zone} (early Oligocene)

Definition. Partial range of the nominate taxon between the last appearance datum (LAD) of Praetenuitella insolita and the LAD of Chiloguembelina cubensis.

It is hoped that this new zone may elucidate the stratigraphic problem at the Eocene/Oligocene boundary in the Southern Indian Ocean.

Berggren (this volume, fig. 5) shows a disconformity that represents a hiatus of 5 m.y. (a possible duration of at least two zones!). However, at Site 749 (Schlich, Wise, et al., 1989), apart from 1\% volcanic ash and some oysters, there is very little evidence for a break and, although sedimentation is very slow, the curve is smooth and the lithology is homogeneous nannofossil ooze.

Furthermore, at Site 748 , there is no unconformity and the boundary is defined by the LAD of Globigerinatheka index. However, this event is apparently 2 m.y. older at Antarctic Site 689 (Stott and Kennett, 1990). Thus, this datum appears to be unreliable in high latitudes and indicates the need for more accurate definition of this major boundary. Although of small size, the tenuitellids are recognizable under the light microscope and provide a rapid age determination for the Eocene/Oligocene boundary. 


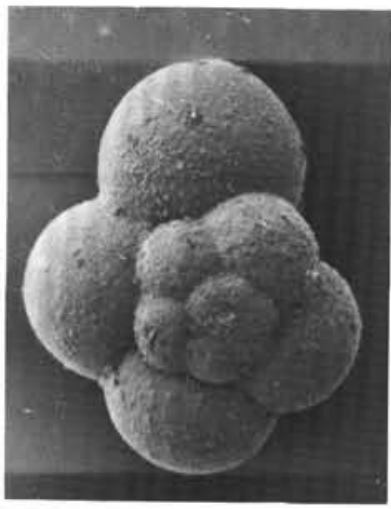

1

$50 \mu \mathrm{m}$

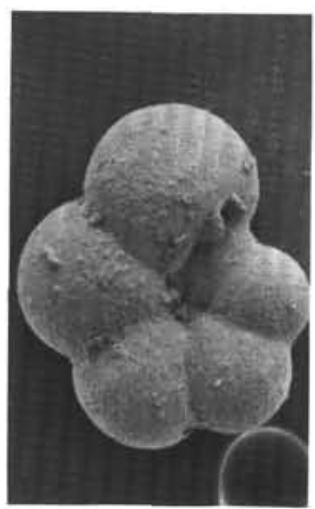

2

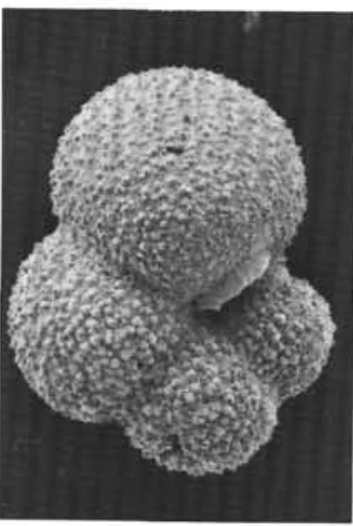

$50 \mu \mathrm{m}$
6

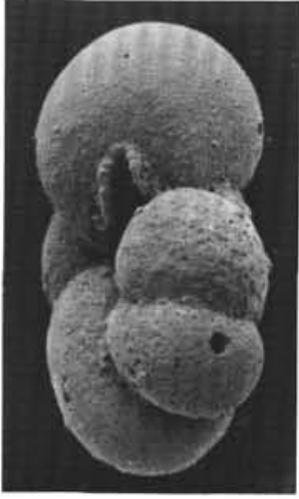

$50 \mu \mathrm{m}$

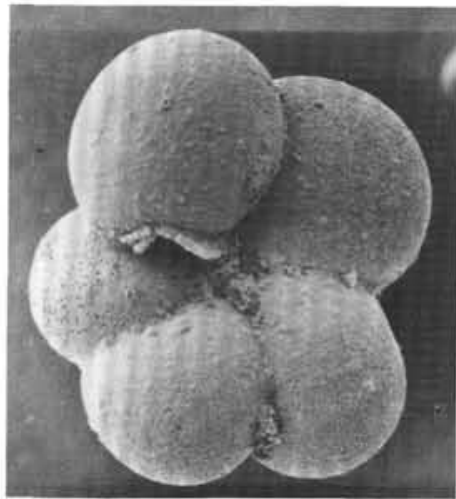

4

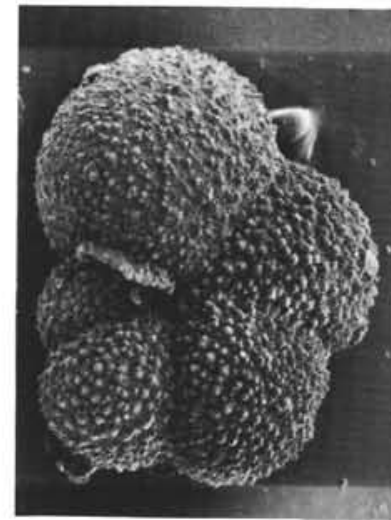

5

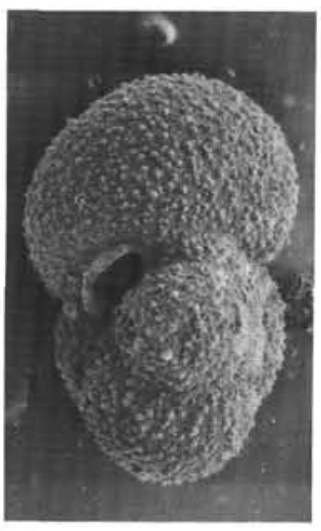

$50 \mu \mathrm{m}$

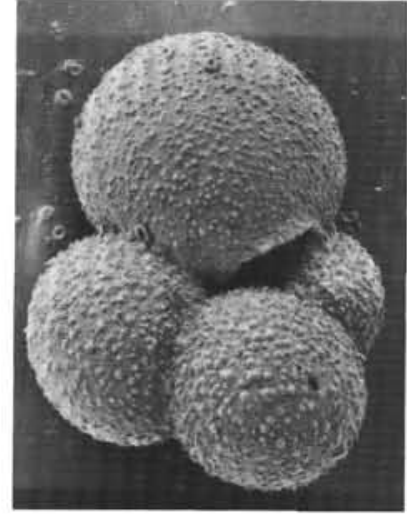

8

$50 \mu \mathrm{m}$

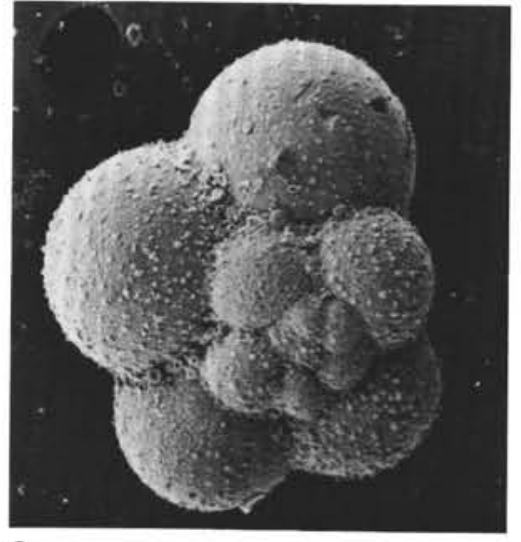

9
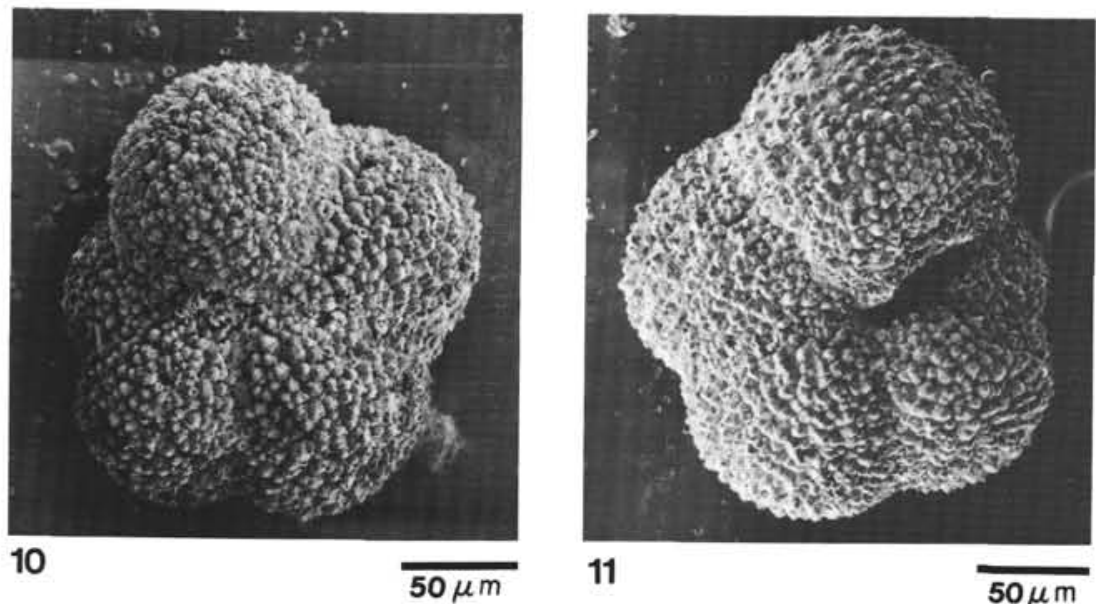

$50 \mu \mathrm{m}$

$50 \mu \mathrm{m}$

Plate 1. 1-3. Praetenuitella insolita; (1) Sample 120-749B-3H-2, 50-53 cm, spiral view; (2) Sample 120-749B-3H-2, 50-53 cm, umbilical view; (3) Sample 120-749B-3H-2, 50-53 cm, peripheral view. 4. Praetenuitella praegemma, Sample 120-749B-3H-2, 50-53 cm, umbilical view. 5. Tenuitella gemma, Sample 120-747A-15H-CC, 0-3 cm, umbilical view. 6-7. Tenuitella gemma, Sample 120-749B-2H-CC, 0-3 cm, umbilical view; (7) same specimen, peripheral view. 8. Specimen intermediate between Tenuitella mundo and Tenuitellinata juvenilis, Sample 120-749B-2H-CC, 0-3 cm, umbilical view. 9-10. Tenuitellinata angustiumbilicata; (9) Sample 120-749B-2H-CC, 0-3 cm, spiral view; (10) Sample 120-747A-16H-CC, 3-6 cm, umbilical view. 11. Specimen intermediate between Tenuitellinata angustiumbilicata and $T$. pseudoedita, Sample 120-747A-14H-1, 105-107 cm, umbilical view. 


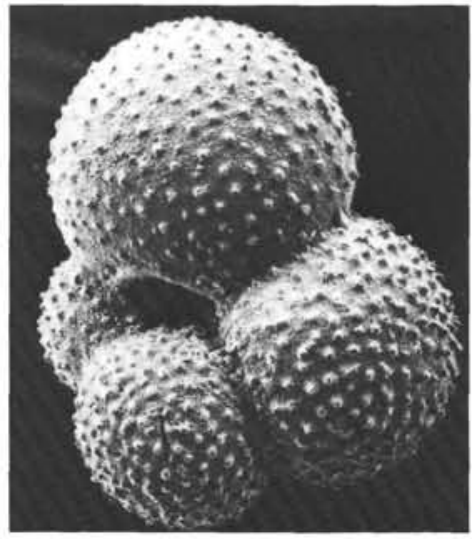

1

$50 \mu \mathrm{m}$

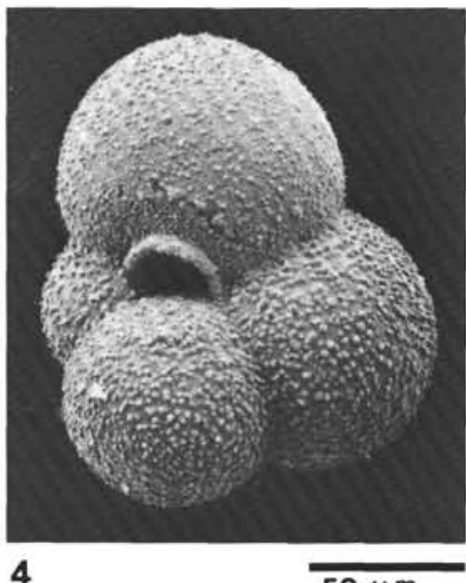

$50 \mu \mathrm{m}$

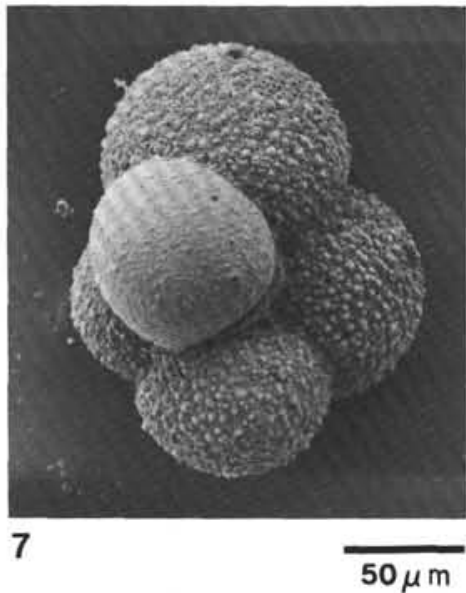

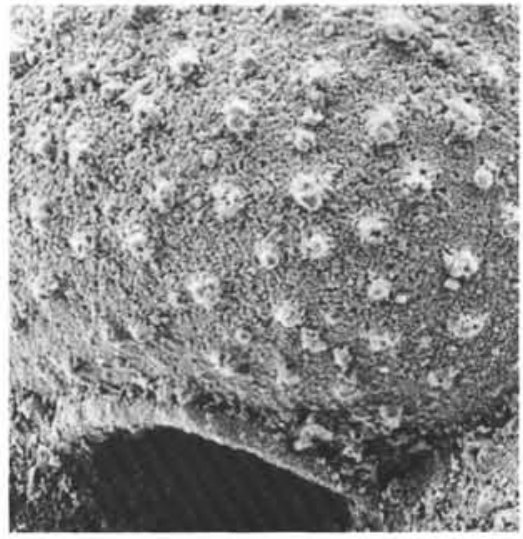

2

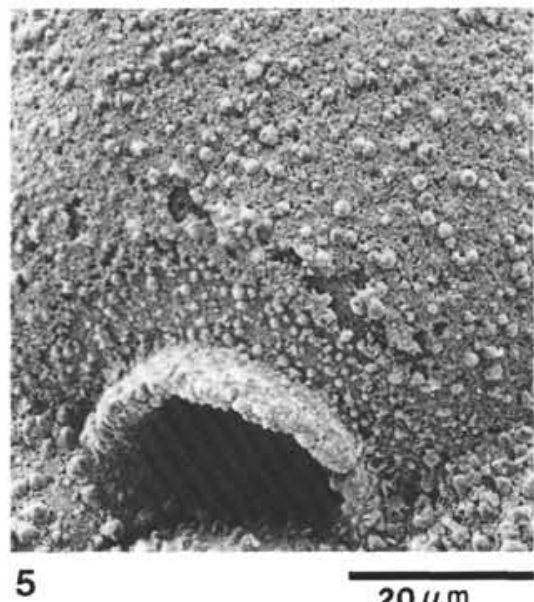

$20 \mu \mathrm{m}$

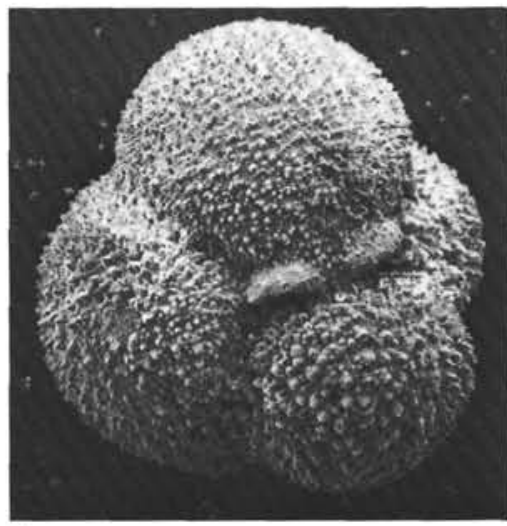

8
$20 \mu \mathrm{m}$

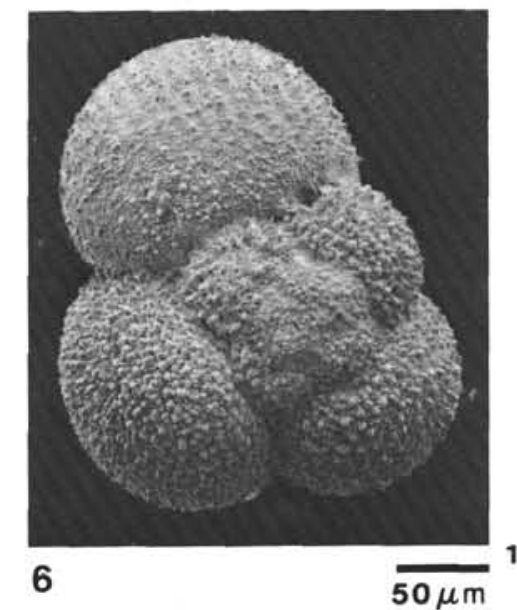

$50 \mu \mathrm{m}$

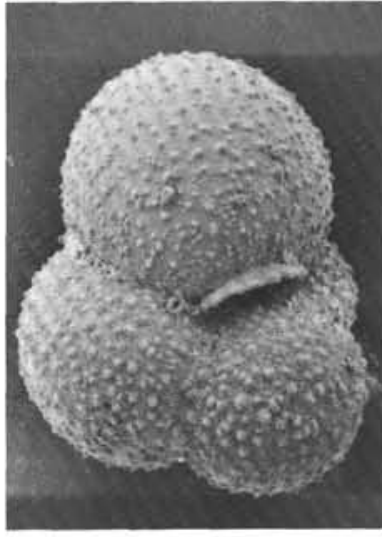

3

$50 \mu \mathrm{m}$

Plate 2. 1-2. Globigerina bulloides antarctica; (1) Sample 120-747A-7H-4, 52-55 cm, umbilical view; (2) same specimen, enlarged to show the spinose wall. 3-6. Tenuitellinata juvenilis; (3) Sample 120-749B-2H-2, 50-53 cm, umbilical view; (4) Sample 120-747A-4H-6, $50-53 \mathrm{~cm}$, umbilical view; (5) same specimen, enlarged to show the microperforate, pustulate wall; (6) Sample 120-747A-11H-4, 50-53 $\mathrm{cm}$, spiral view. 7. Globigerinita boweni, Sample 120-749B-1H-2, 50-53 cm, umbilical view. 8. Globigerinata naparimaensis, Sample $120-747 \mathrm{~A}-11 \mathrm{H}-4,50-53 \mathrm{~cm}$, apertural view. 9. Globigerinita glutinata, Sample 120-747A-9H-5, 50-53 cm, umbilical view. 


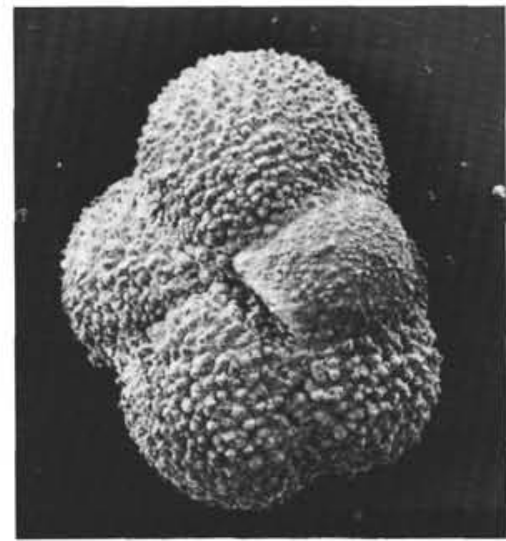

1

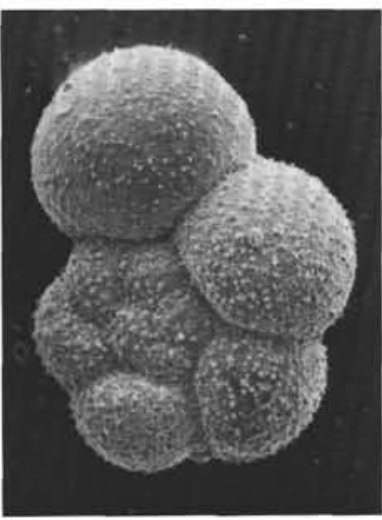

4

$50 \mu \mathrm{m}$

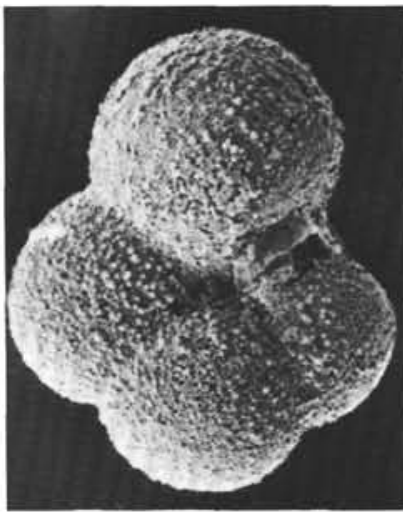

8 $\overline{50 \mu \mathrm{m}}$
5

9

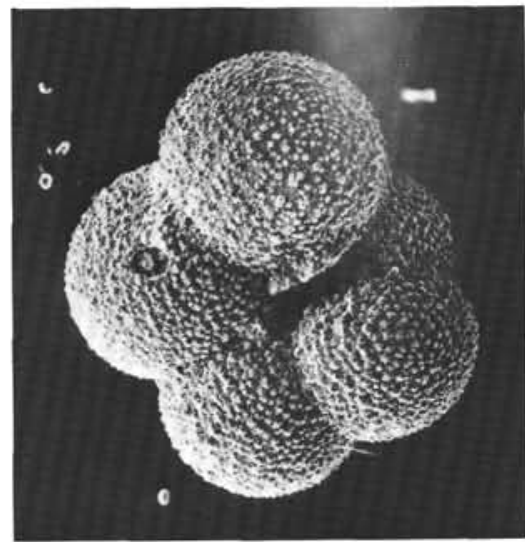

2
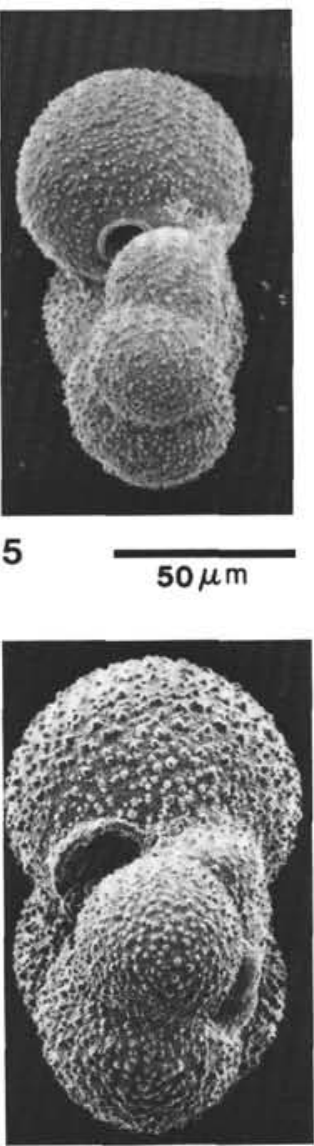
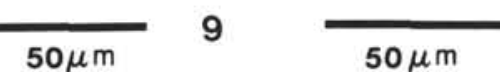

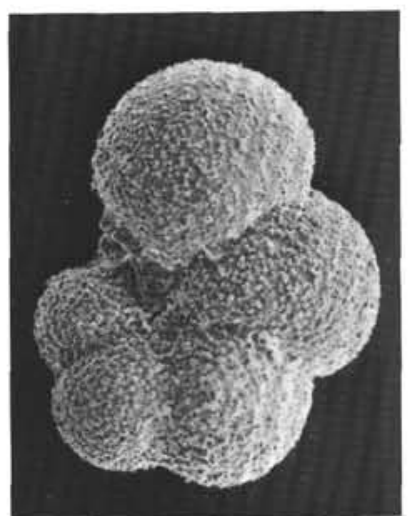

$50 \mu \mathrm{m}$

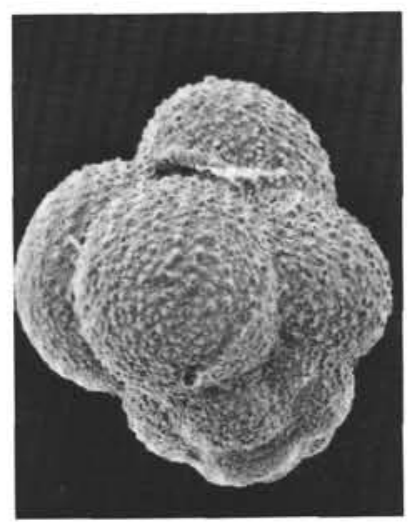

10

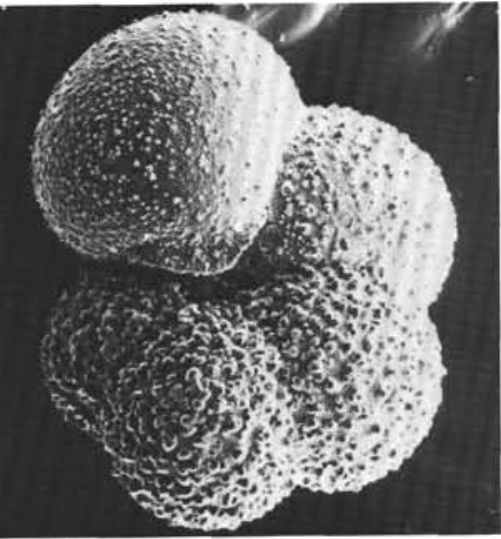

3

$50 \mu \mathrm{m}$

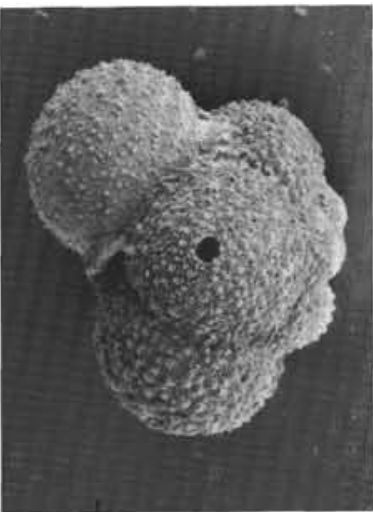

7

$50 \mu \mathrm{m}$

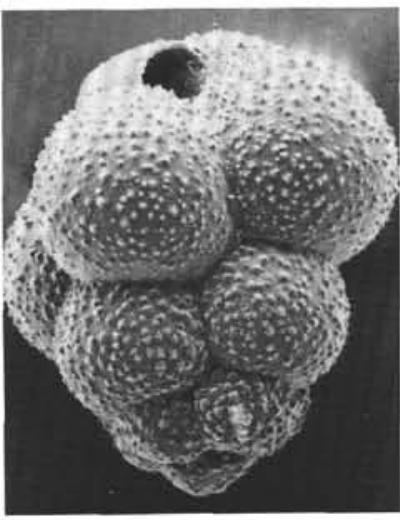

11

$50 \mu \mathrm{m}$

Plate 3. 1. Globigerinita praestainforthi, Sample $120-747 \mathrm{~A}-14 \mathrm{H}-3,55-58 \mathrm{~cm}$, umbilical view. 2-3. Tenuitellinata pseudoedita; (2) Sample 120-747A-9H-6, 50-53 cm, umbilical view; (3) Sample 120-747A-8H-4, 55-58 cm, umbilical view. 4-6. Tenuitella minutissima; (4) Sample 120-747A-12H-4, 50-53 cm, spiral view; (5) Sample 120-747A-12H-4, 50-53 cm, peripheral view; (6) Sample 120-747A$12 \mathrm{H}-4,50-53 \mathrm{~cm}$, umbilical view. 7. Specimen intermediate between Tenuitellinata juvenilis and Tenuitellinata uvula, Sample 120-747A-2H-2, 50-53 cm, side view. 8-9. Tenuitella clemenciae; (8) Sample 120-747A-11H-2, 50-53 cm, umbilical view; (9) Sample 120-747A-8H-6, 50-53 cm, peripheral view. 10-11. Tenuitellinata uvula; (10) Sample 120-747A-10H-CC, 0-3 cm, side view; (11) Sample 120-747A-1H-3, $50-53 \mathrm{~cm}$, side view. 


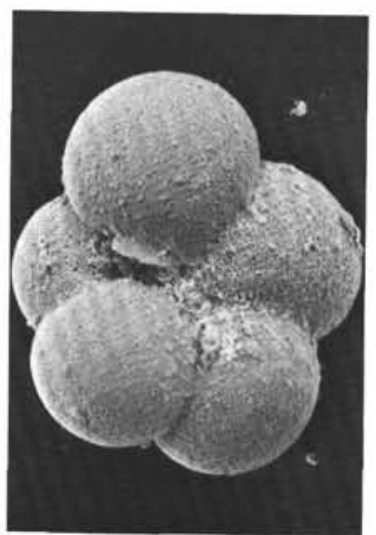

1

$50 \mu \mathrm{m}$
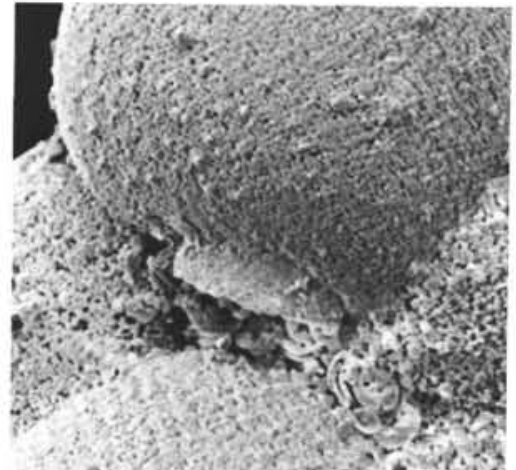

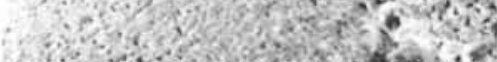

2

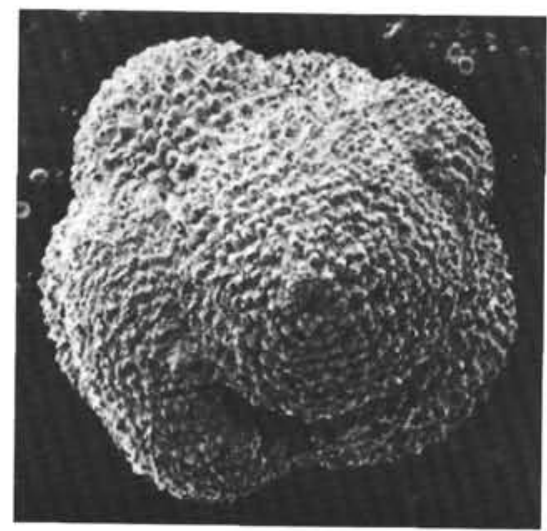

5

$50 \mu \mathrm{m}$

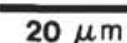

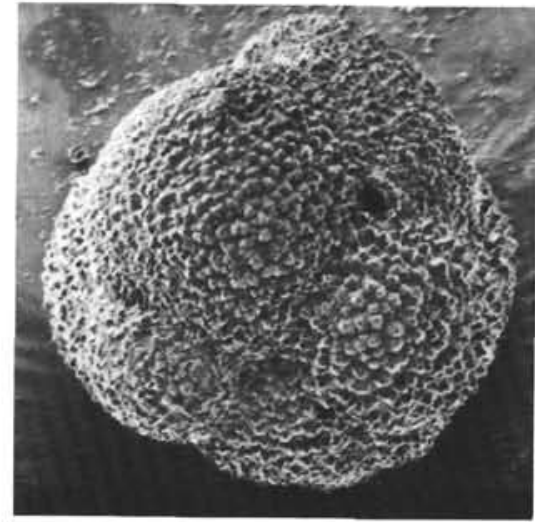

6

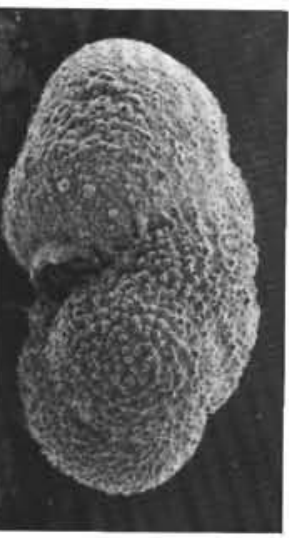

$50 \mu \mathrm{m}$

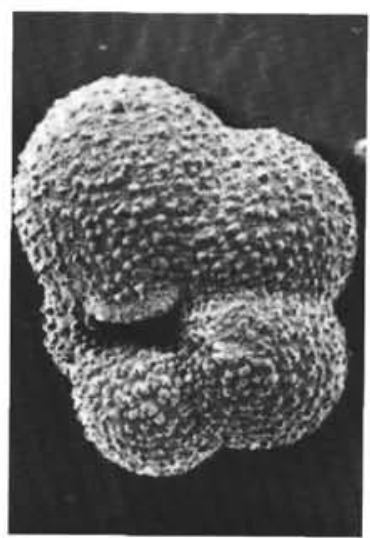

10
$50 \mu \mathrm{m}$

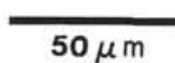

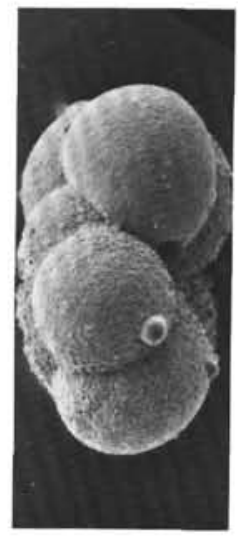

$\overline{20 \mu \mathrm{m}}$

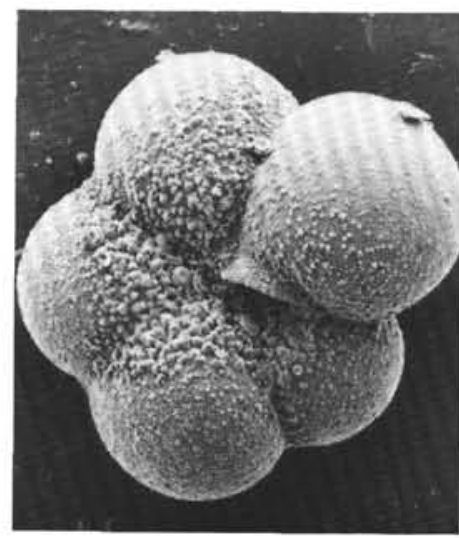

4

$50 \mu \mathrm{m}$

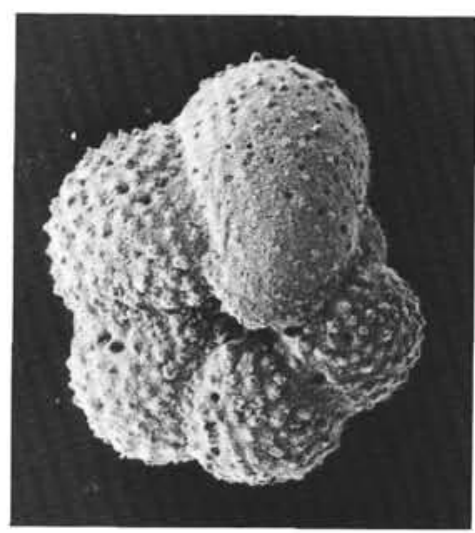

7

$50 \mu \mathrm{m}$

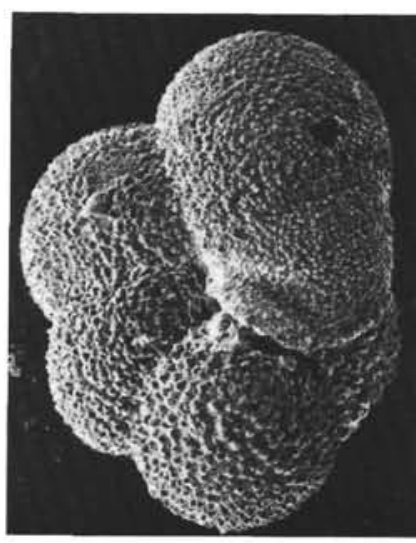

11

$50 \mu \mathrm{m}$

Plate 4. 1-4. Tenuitellinata selleyi n. sp.; (1) Sample 120-747A-11H-CC, 0-3 cm, holotype, umbilical view; (2) holotype enlarged to show the anteriointraumbilical aperture and smooth wall; (3) Sample 120-747A-12H-2, 50-53 cm, paratype, peripheral view; (4) Sample $120-747 \mathrm{~A}-8 \mathrm{H}-6,50-53 \mathrm{~cm}$, paratype, umbilical view; note the pustules restricted to the umbilical area. 5-6. Globigerinatella sp.; (5) Sample 120-747A-11H-2, 50-53 cm, side view; (6) same specimen, side view. 7. Turborotalita quinqueloba, Sample 120-747A-5H-CC, $0-3 \mathrm{~cm}$, umbilical view, showing the spinose wall. 8-11. Tenuitella jamesi $\mathrm{n}$. sp.; (8) Sample 120-747A-11H-CC, 0-3 cm, holotype, umbilical view; (9) holotype, peripheral view; (10) Sample 120-747A-8H-4, 55-58 cm, paratype, umbilical view; (11) Sample $120-747 \mathrm{~A}-10 \mathrm{H}-4,52-55 \mathrm{~cm}$, paratype, umbilical view. 